\title{
The leptonic future of the Higgs
}

\author{
Gauthier Durieux, ${ }^{a}$ Christophe Grojean, ${ }^{a, b, 1}$ Jiayin $\mathbf{G u}^{a, c}$ and Kechen Wang ${ }^{a, c}$ \\ ${ }^{a} D E S Y$, \\ Notkestraße 85, D-22607 Hamburg, Germany \\ ${ }^{b}$ Institut für Physik, Humboldt-Universität zu Berlin, \\ Newtonstraße 15, D-12489 Berlin, Germany \\ ${ }^{c}$ Center for Future High Energy Physics, Institute of High Energy Physics, \\ Chinese Academy of Sciences, \\ 19B YuquanLu, Beijing 100049, China \\ E-mail: gauthier.durieux@desy.de, christophe.grojean@desy.de, \\ jiayin.gu@desy.de, kechen.wang@desy.de
}

ABSTRACT: Precision study of electroweak symmetry breaking strongly motivates the construction of a lepton collider with center-of-mass energy of at least $240 \mathrm{GeV}$. Besides Higgsstrahlung $\left(e^{+} e^{-} \rightarrow h Z\right)$, such a collider would measure weak boson pair production $\left(e^{+} e^{-} \rightarrow W W\right)$ with an astonishing precision. The weak-boson-fusion production process $\left(e^{+} e^{-} \rightarrow \nu \bar{\nu} h\right)$ provides an increasingly powerful handle at higher center-of-mass energies. High energies also benefit the associated top-Higgs production $\left(e^{+} e^{-} \rightarrow t \bar{t} h\right)$ that is crucial to constrain directly the top Yukawa coupling. The impact and complementarity of differential measurements, at different center-of-mass energies and for several beam polarization configurations, are studied in a global effective-field-theory framework. We define a global determinant parameter (GDP) which characterizes the overall strengthening of constraints independently of the choice of operator basis. The reach of the CEPC, CLIC, FCC-ee, and ILC designs is assessed.

Keywords: Beyond Standard Model, Effective Field Theories, Higgs Physics

ARXIV EPRINT: 1704.02333

\footnotetext{
${ }^{1}$ On leave from Institució Catalana de Recerca i Estudis Avançats, 08010 Barcelona, Spain.
} 


\section{Contents}

1 Introduction 1

2 Effective-field-theory framework $\quad 3$

$\begin{array}{lll}3 & \text { Measurements and fit } & 7\end{array}$

3.1 Higgsstrahlung production 8

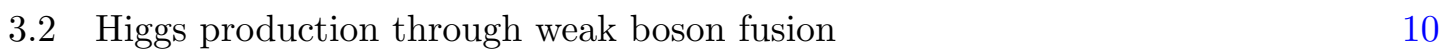

$\begin{array}{lll}3.3 & \text { Higgs production in association with tops } & 11\end{array}$

$\begin{array}{ll}3.4 & \text { Weak-boson pair production } \\ & 11\end{array}$

$\begin{array}{lll}3.5 & \text { Global fit and determinant parameter } & 14\end{array}$

$\begin{array}{lll}4 & \text { Results } & 15\end{array}$

5 Conclusions $\quad 23$

$\begin{array}{ll}\text { A Effective-field-theory parameter definitions } & 25\end{array}$

$\begin{array}{ll}\text { B Measurement inputs } & 27\end{array}$

$\begin{array}{lll}\text { C Additional figures } & 32\end{array}$

D Numerical expressions for the observables $\quad 33$

$\begin{array}{ll}\text { E Numerical results of the global fit } & 41\end{array}$

\section{Introduction}

With the discovery of a scalar whose properties are compatible with that of the standard model (SM) Higgs boson, the first run of the LHC has found an essential ingredient for the deep understanding of matter and has revealed a fascinating and complex structure of the vacuum. As its second run is proceeding at an increased center-of-mass energy, no unambiguous sign of new physics (NP) has been found yet. Direct exploration of this energy frontier will continue for a couple of decades but a detailed understanding of electroweak symmetry breaking and the indirect search for NP via precision measurement would benefit from the cleaner environment of a lepton collider. An $e^{+} e^{-}$machine running at a centerof-mass energy of 240-250 GeV, close to the maximum of the $e^{+} e^{-} \rightarrow h Z$ Higgsstrahlung cross section would indeed determine the Higgs couplings with exquisite precision. Several proposals of such Higgs factories have been made, including the Circular Electron Positron Collider (CEPC) in China [1], the Future Circular Collider with $e^{+} e^{-}$(FCC-ee) at CERN, previously known as TLEP [2], and the International Linear Collider (ILC) in Japan [3]. 
The Compact Linear Collider (CLIC) at CERN [4] could also run at higher center-of-mass energies. The Higgs coupling measurements have been widely studied in the corresponding design studies through global fits in the so-called kappa framework [5].

As new physics is being constrained to lie further and further above the electroweak scale, the description of its effects at future lepton colliders seems to fall in a low-energy regime. Effective field theories (EFTs) therefore look like prime exploration tools [6-11]. Given that the parity of an operator dimension is that of $(\Delta B-\Delta L) / 2$ [12], all operators conserving baryon and lepton numbers are of even dimension:

$$
\mathcal{L}_{\mathrm{EFT}}=\mathcal{L}_{\mathrm{SM}}+\sum_{i} \frac{c_{i}^{(6)}}{\Lambda^{2}} \mathcal{O}_{i}^{(6)}+\sum_{j} \frac{c_{j}^{(8)}}{\Lambda^{4}} \mathcal{O}_{j}^{(8)}+\cdots
$$

where $\Lambda$ is a mass scale and $c_{i}^{(d)}$ are the dimensionless coefficients of the $\mathcal{O}_{i}^{(d)}$ operators of canonical dimension $d$. The standard-model effective field theory (SMEFT) allows for a systematic exploration of the theory space in direct vicinity of the standard model, encoding established symmetry principles. As a genuine quantum field theory, its predictions are also perturbatively improvable. It therefore relies on much firmer theoretical bases than the kappa framework. While very helpful in illustrating the precision reach of Higgs measurements, the latter can in particular miss interactions of Lorentz structure different from that of the standard model, or correlations deriving from gauge invariance, notably between Higgs couplings to different gauge bosons.

Many effective-field-theory studies have been performed, for Higgs measurements at LHC [13-17], electroweak (EW) precision observables at LEP [18-22], diboson measurements at both LEP [23] and LHC [24, 25], or the combination of measurements in several sectors [26, 27]. Among the studies performed in the context of future Higgs factories [2836], many estimated constraints on individual dimension-six operators. A challenge related to the consistent use of the EFT framework is indeed the simultaneous inclusion of all operators up to a given dimension. It is required for this approach to retain its power and generality. As a result, various observables have to be combined to constrain efficiently all directions of the multidimensional space of effective-operator coefficients. The first few measurements included bring the more significant improvements by lifting large approximate degeneracies. Besides Higgsstrahlung production and decay rates in different channels, angular distributions contain additional valuable information [29, 31]. Our knowledge about differential distributions could also be exploited more extensively through statistically optimal observables [37, 38]. Higgs production through weak-boson fusion provides complementary information of increasing relevance at higher center-of-mass energies. Direct constraints on the top Yukawa coupling can moreover only be obtained through Higgs production in association with a pair of tops. Measurements at $\sqrt{s}=350 \mathrm{GeV}$ and above can thus be very helpful. As the sensitivities to operator coefficients can vary with $\sqrt{s}$, these higher-energy runs would also constrain different directions of the parameter space and therefore resolve degeneracies. Beam polarization, more easily implemented at linear colliders, could be similarly helpful. Finally, the Higgs and anomalous triple gauge cou- 
plings (aTGCs) are related in a gauge-invariant EFT, and a subset of operators relevant for Higgs physics can be efficiently bounded through diboson production $e^{+} e^{-} \rightarrow W W[17,23]$.

We parametrize deviations from the standard-model in the processes enumerated above through dimension-six operators, in the so-called Higgs basis [39]. Translation to other bases is however straightforward. Our assumption of perfectly standard-model-like electroweak precision measurements is more easily implemented in that framework. No deviation in the gauge-boson couplings to fermions, or $W$ mass is permitted. Given the poor sensitivity expected for the Yukawa couplings of lighter fermion, we only allow for modifications of the (flavor-conserving) muon, tau, charm, bottom, and top ones. Neither $\mathrm{CP}$-violating, nor fermion dipole operators are considered. The potential impact of these assumptions on our results is carefully discussed in the text. A global effective-field-theory analysis is then performed, in a twelve-dimensional parameter space, assuming that measurements coincide with their SM predictions. Prospects for the different machines are discussed in view of their respective design and run plan.

The rest of this paper is organized as follows. In section 2, we lay down the EFT framework used. In section 3, we detail the observables included in our study. The results of the global fits are shown in section 4 . The reach of the different colliders is summarized in figure 7. Our conclusions are drawn in section 5. Further details are provided in the appendix. We define our twelve effective-field-theory parameters and provide their expressions in the SILH' basis in appendix A. Additional information about the measurement inputs is provided in appendix B. Supplementary figures and results are available in appendix C. In appendix D, we provide numerical expressions for the observables used in terms of our twelve effective-field-theory parameters. Finally, the numerical results of the global fits are tabulated in appendix E. They could be used to set limits on specific models while accounting for the correlations in the full twelve-dimensional parameter space.

\section{Effective-field-theory framework}

A global effective-field-theory treatment of any process requires to consider simultaneously all contributing operators appearing in a complete basis, up to a given dimension. Assuming baryon and lepton number conservations, we restrict ourselves to dimension-six operators. As mentioned in the introduction, we would like to model the following processes:

- Higgsstrahlung production: $e^{+} e^{-} \rightarrow h Z$ (rates and distributions),

followed by Higgs decays in various channels,

- Higgs production through weak-boson fusion: $e^{+} e^{-} \rightarrow \nu \bar{\nu} h$,

- Higgs production in association with top quarks: $e^{+} e^{-} \rightarrow t \bar{t} h$,

- weak-boson pair production: $e^{+} e^{-} \rightarrow W W$ (rate and distributions).

Several combinations of operators affecting these processes are however well constrained by other measurements. As discussed in section 3.4, electroweak precision observables could be constrained to a sufficient level, although this remains to be established explicitly. 
At leading order, CP-violating operators give no linear contribution to the Higgs rates but could manifest themselves in angular asymmetries [29, 31]. They could moreover be well constrained by dedicated searches. Under restrictive assumptions, indirect constraints arising from EDM experiments [40-42] for instance render Higgs CP-violating asymmetries inaccessible at future colliders [31], even thought some room may be left in the CP violating Yukawa of the charm and bottom quarks for which the direct and indirect bounds are not that restrictive [43]. It is also possible for CP violating Yukawa couplings of heavy flavor leptons to evade the constraints from EDM experiments which could be probed in Higgs decays [44]. As a first working hypothesis, we thus assume electroweak and CP-violating observables are perfectly constrained to be standard-model like.

Throughout this paper, we only retain the interferences of effective-field-theory amplitudes with standard-model ones. The squares of amplitudes featuring a dimension-six operator insertion are discarded. They are formally of the same $c^{2} / \Lambda^{4}$ order as the interferences of dimension-eight operators with standard-model amplitudes. The relative importance of these two kinds of $c^{2} / \Lambda^{4}$ contributions can however not be determined without assuming a definite power counting or referring to a specific model. Nevertheless, thanks to the high precision to which most observables are measured at lepton colliders that collect large amount of integrated luminosity in clean environments, we generically expect the discarded terms to have small impact on our results. The percent-level measurement of an observable of schematic

$$
\frac{O}{O_{\mathrm{SM}}}=1+\mathcal{O}(1) \frac{c E^{2}}{\Lambda^{2}}+\mathcal{O}(1)\left(\frac{c E^{2}}{\Lambda^{2}}\right)^{2}
$$

effective-field-theory dependence (where $E$ is a typical energy scale) will for instance constrain $c E^{2} / \Lambda^{2}$ at the percent level. The quadratic term then only induces a relative percent-level correction to this limit. In specific cases, the interference of dimension-six operators with standard-model amplitudes can however suffer accidental suppressions. This could invalidate the naïve hierarchy above between linear and quadratic terms. Helicity selection rules [45] can for instance cause significant suppressions of the linear contribution compared to the quadratic one, at energies higher than electroweak mass scales. If the standard model and dimension-six operators give rise to amplitudes with electroweak bosons of different helicities, their interference is expected to scale as $c m_{V}^{2} / \Lambda^{2}$. A measurement of $O / O_{\mathrm{SM}}$ with precision $x$ would still imply a limit of order $x$ on $\mathrm{cm}_{V}^{2} / \Lambda^{2}$ at low energies but this bound would receive corrections scaling as $x E^{4} / m_{V}^{4}$ for increasing $E$. Given $m_{V}$ of order $100 \mathrm{GeV}$, only measurements of $10^{-2}, 10^{-3}, 10^{-4}, 10^{-5}$ and $10^{-6}$ precisions at least are roughly expected to be dominated by linear effective-field-theory contributions at 250 , 500, 1000, 1400 and $3000 \mathrm{GeV}$ energies, respectively. We will comment further on accidental suppressions and on their possible impact on our results in section 4. Light fermion dipole operators also have interferences with standard-model amplitudes that suffer drastic mass suppressions. As a consequence, their dominant effects arise at the $c^{2} / \Lambda^{4}$ level. We however leave the study of this family of operators for future work.

Under the above assumptions, together with flavor universality, it was shown that there are 10 independent combinations of operators that contribute to Higgs (excluding 
its self coupling) and TGC measurements $[13,14,16,23] .{ }^{1}$ We however lift the flavor universality requirement and treat separately the top, charm, bottom, tau, and muon Yukawa couplings. No flavor violation is allowed and we refer to refs. [46-48] for studies of the possible means to probe the light-fermion Yukawas at present and future experiments. In total, 12 degrees of freedom are thus considered. While all non-redundant basis are equivalent, we find the Higgs basis [39] particularly convenient. It is defined in the broken electroweak phase and therefore closely related to experimental observables. Distinguishing the operators contributing to electroweak precision measurements from the ones of Higgs and TGC measurements is also straightforward in this basis. The parameters we use are:

$$
\delta c_{Z}, \quad c_{Z Z}, \quad c_{Z \square}, \quad c_{\gamma \gamma}, \quad c_{Z \gamma}, \quad c_{g g}, \quad \delta y_{t}, \quad \delta y_{c}, \quad \delta y_{b}, \quad \delta y_{\tau}, \quad \delta y_{\mu}, \quad \lambda_{Z}
$$

Their exact definitions as well as a correspondence map to the SILH' basis of gaugeinvariant dimension-six operators can be found in appendix A. The numerical expressions of the various observables we use as functions of these parameters are given in appendix D.

Compared to the widely-used kappa framework, an important feature of this effective field theory is the appearance of Higgs couplings with Lorentz structures differing from SM ones. In addition to $\delta c_{Z} h Z_{\mu} Z^{\mu}$ which modifies an existing SM coupling, the $c_{Z Z} h Z_{\mu \nu} Z^{\mu \nu}$ and $c_{Z \square} h Z_{\mu} \partial_{\nu} Z^{\mu \nu}$ interactions are for instance also generated by gaugeinvariant dimension-six operators. The $e^{+} e^{-} \rightarrow h Z$ rate, at a given center-of-mass energy and for a fixed beam polarization, depends on one combination of these parameters. Runs at various energies, with different beam polarizations, as well as additional measurements are therefore crucial to constrain all other orthogonal directions. Measurements at higher center-of-mass energies have an enhanced sensitivity to $c_{Z Z}$ and $c_{Z \square}$. Angular asymmetries in $e^{+} e^{-} \rightarrow h Z$, weak-boson-fusion production rate, weak-boson pair production, or the $h \rightarrow Z Z^{*}$ and $h \rightarrow W W^{*}$ decays, each play a role. The measurement of the $h \rightarrow Z \gamma$ decay is crucial too. The $c_{Z \gamma}$ coupling which contributes to the Higgsstrahlung process otherwise remains loosely constrained and weakens the whole fit.

The treatment of the $h \rightarrow g g, \gamma \gamma$, and $Z \gamma$ decays requires some special attention. Given that they are loop-level generated in the standard model, one may wish to include their loop-level dependence in effective parameters like $\delta y_{t}, \delta y_{b}, \delta c_{W}$ which rescale standardmodel interactions, or $c_{Z Z}, c_{Z \square}$, etc. which do not. Complete effective-field-theory results at that order are however not currently available for the above processes (see ref. [49] for the treatment of $h \rightarrow \gamma \gamma$ ). The computation of next-to-leading-order effective-field-theory contributions to processes that are not loop-level generated in the standard model would also be needed to ensure a consistent global treatment. Misleading results can otherwise be obtained. Let us illustrate this point with the dependence of the $h \rightarrow \gamma \gamma$ partial width on $c_{\gamma \gamma}$ and $\delta y_{t}$, at tree- and loop-level, respectively. The Higgsstrahlung, weak-boson fusion, and weak-boson pair production processes also depend at tree level on $c_{\gamma \gamma}$ and receive loop corrections proportional to $\delta y_{t}$. A combination of these two parameters similar to the one entering in the $h \rightarrow \gamma \gamma$ partial width may moreover be expected. Including the

\footnotetext{
${ }^{1}$ Refs. [26, 32, 35] additionally set lepton and down-type Yukawa couplings equal while ref. [17] focuses on third-generation fermions instead of assuming flavor universality.
} 
dependence of this partial width on $\delta y_{t}$, but not that of the $e^{+} e^{-} \rightarrow h Z, e^{+} e^{-} \rightarrow \nu \bar{\nu} h$, and $e^{+} e^{-} \rightarrow W W$ cross sections, one would artificially render their constraints orthogonal. Tight bounds on $\delta y_{t}$ would then be obtained. Consistently including all one-loop dependences on these parameters might however still leave a combination of $c_{\gamma \gamma}$ and $\delta y_{t}$ at least nearly unconstrained. To avoid such a pitfall, we choose not to include any loop-level dependence on effective-field-theory parameters in the $h \rightarrow \gamma \gamma$ and $Z \gamma$ partial widths. Once direct constraints on the top Yukawa coupling (from the LHC or from $e^{+} e^{-} \rightarrow t \bar{t} h$ ) are included, we however checked that including the whole loop dependence of the $h \rightarrow \gamma \gamma$ branching fraction has only marginal effects on our results. ${ }^{2}$ For our purpose, it is on the contrary safe to account for the loop-level $\delta y_{t}$ and $\delta y_{b}$ dependences of the $h \rightarrow g g$ partial width. It remains to be examined whether the loop-level dependence on $\delta y_{t}$ in processes measured at lepton collider, below the $t \bar{t} h$ threshold, could serve to improve on the highluminosity LHC constraints. A similar question, asked for the trilinear Higgs coupling [51] could be further investigated.

Absorbing also, for convenience, a standard-model normalization factor into barred effective parameters, we thus obtain:

$$
\frac{\Gamma_{\gamma \gamma}}{\Gamma_{\gamma \gamma}^{\mathrm{SM}}} \simeq 1-2 \bar{c}_{\gamma \gamma}, \quad \frac{\Gamma_{Z \gamma}}{\Gamma_{Z \gamma}^{\mathrm{SM}}} \simeq 1-2 \bar{c}_{Z \gamma},
$$

and

$$
\frac{\Gamma_{g g}}{\Gamma_{g g}^{\mathrm{SM}}} \simeq 1+2 \bar{c}_{g g}^{\mathrm{eff}} \simeq 1+2 \bar{c}_{g g}+2.10 \delta y_{t}-0.10 \delta y_{b},
$$

at the linear order. Compared to the standard Higgs-basis effective parameters, our normalization is the following:

$$
\bar{c}_{\gamma \gamma} \simeq \frac{c_{\gamma \gamma}}{8.3 \times 10^{-2}}, \quad \bar{c}_{Z \gamma} \simeq \frac{c_{Z \gamma}}{5.9 \times 10^{-2}}, \quad \bar{c}_{g g} \simeq \frac{c_{g g}}{8.3 \times 10^{-3}} .
$$

We will sometimes display results in terms of the $\bar{c}_{g g}^{\text {eff }}$ parameter that is directly probed by the $h \rightarrow g g$ branching fraction. It is particularly informative to do so when $c_{g g}$ and $\delta y_{t}$ are only poorly constrained individually.

Measurement of the $h \rightarrow Z Z^{*}$ rate relies on its fermionic decay products and has some sensitivity on $c_{\gamma \gamma}$ and $c_{Z \gamma}$, in addition to $\delta c_{Z}, c_{Z Z}$ and $c_{Z \square}$. Higgs decays to off-shell photons can indeed produce the same final state. Each fermionic decay channel actually has a somewhat different sensitivity which depends strongly on the invariant mass of fermion pairs. Loosened cuts would provide increased sensitivities to $c_{\gamma \gamma}$ and $c_{Z_{\gamma}}$ [52]. ${ }^{3}$ For simplicity, we however neglect the contributions of those two effective-field-theory parameters to $h \rightarrow Z Z^{*}$. Standard invariant mass cuts together with the constraints on $c_{\gamma \gamma}$ and $c_{Z \gamma}$ arising from the direct measurements of $h \rightarrow Z \gamma$ and $h \rightarrow \gamma \gamma$ decays should be sufficient to limit the impact of this approximation on our results.

The standard-model effective field theory we use specifically assumes the absence of new states below the electroweak scale. It does therefore not account for possible invisible decays

\footnotetext{
${ }^{2}$ We used the numerical expressions derived from the results of ref. [49] in the appendix of ref. [50].

${ }^{3}$ See also ref. [53] for a recent EFT study of the Higgs decay into four charged leptons exploiting both the rates and kinematic distributions.
} 
of the Higgs. The corresponding branching fraction would nevertheless be significantly constrained at future lepton colliders. An integrated luminosity of $5 \mathrm{ab}^{-1}$ collected at $240 \mathrm{GeV}$ would for instance bound $\sigma(h Z) \times \mathrm{BR}(h \rightarrow$ inv $)$ to be smaller than $0.28 \%$ of $\sigma(h Z)$ at the $95 \%$ CL [1]. Other exotic Higgs decays not modeled in a SMEFT framework would also be constrained very well at future lepton colliders [54]. We do therefore not expect an effective field theory modified to include such decays to lead to results widely different from the ones we obtain.

\section{Measurements and fit}

To the best of our knowledge, the most updated run plans of each machine are the following:

- According to its preCDR, the CEPC would collect $5 \mathrm{ab}^{-1}$ of integrated luminosity at $240 \mathrm{GeV}$. Recently, the reference circumference of its tunnel has been fixed to $100 \mathrm{~km}$ [55]. A run at $350 \mathrm{GeV}$ could therefore be envisioned. The luminosity to expect at that center-of-mass energy however depends on the machine design and is currently unknown. To study the impact of the measurements at $350 \mathrm{GeV}$, we take a conservative benchmark value of $200 \mathrm{fb}^{-1}$ and explore a larger range in section 4 .

- The CDR of the FCC-ee project is expected by the year 2018 [56] and will supersede the TLEP white paper [2] that still contains the most recent results on Higgs physics. The latter document, we rely on, assumes that $10 \mathrm{ab}^{-1}$ of data would be collected at $240 \mathrm{GeV}$ and $2.6 \mathrm{ab}^{-1}$ at $350 \mathrm{GeV}$.

- Recent ILC documents suggest that, with a luminosity upgrade, it could collect $2 \mathrm{ab}^{-1}$ at $250 \mathrm{GeV}, 200 \mathrm{fb}^{-1}$ at $350 \mathrm{GeV}$, and $4 \mathrm{ab}^{-1}$ at $500 \mathrm{GeV}$ [57, 58]. This significantly extends the plans presented in its TDR [3]. The updated estimations are adopted in our study. The ILC could also run with longitudinally polarized beams. We follow refs. $[3,58]$ and assume that a maximum polarization of $\pm 80 \%( \pm 30 \%)$ can be achieved for the incoming electron (positron). While collecting $1 \mathrm{ab}^{-1}$ of integrated luminosity at a center-of-mass energy of $1 \mathrm{TeV}$, with $P\left(e^{-}, e^{+}\right)=(-0.8,+0.2)$ polarization, is also considered in the TDR [3], we follow refs. [57, 58] and do not take such a run into account. Nevertheless, results including the $1 \mathrm{TeV}$ measurements of precision quoted in ref. [59] are shown in appendix C.

- Recent ref. [4] proposed that CLIC would collect $100 \mathrm{fb}^{-1}$ at the top threshold, $500 \mathrm{fb}^{-1}$ at $380 \mathrm{GeV}, 1.5 \mathrm{ab}^{-1}$ at $1.5 \mathrm{TeV}$, and $3 \mathrm{ab}^{-1}$ at $3 \mathrm{TeV}$. The more specific study of Higgs measurements of ref. [60] however assumed $500 \mathrm{fb}^{-1}$ at $350 \mathrm{GeV}$, $1.5 \mathrm{ab}^{-1}$ at $1.4 \mathrm{TeV}$ and $2 \mathrm{ab}^{-1}$ at $3 \mathrm{TeV}$. We follow the latter plan in order to make use of its estimations. While the implementation of beam polarization is also likely at CLIC, we follow again ref. [60] and assume unpolarized beams.

In the rest of this section, we summarize the important aspects of each of the measurements we take into account. We detail the assumptions made in the many cases where necessary information is not provided in the literature. The numerical inputs we use are given in appendix B. 


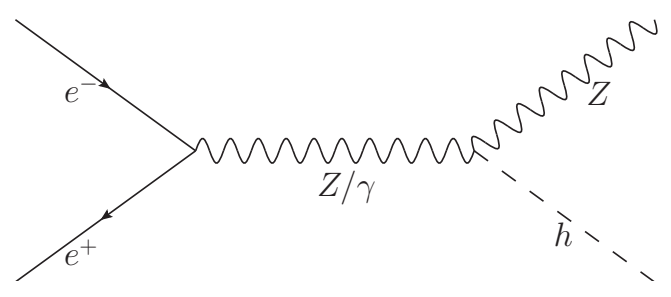

Figure 1. Leading-order contribution to the Higgsstrahlung process, $e^{+} e^{-} \rightarrow h Z$.

\subsection{Higgsstrahlung production}

Rate measurements. The Higgsstrahlung process (see figure 1) dominates the Higgs production modes at lepton colliders below center-of-mass energies of about $450 \mathrm{GeV}$ where weak-boson fusion takes over. Its cross section is maximized around $250 \mathrm{GeV}$ but bremsstrahlung makes it more advantageous for circular colliders to run at $240 \mathrm{GeV}$. At this energy, an integrated luminosity of $5 \mathrm{ab}^{-1}$ would yield about $1.06 \times 10^{6}$ Higgses. At $250 \mathrm{GeV}, 2 \mathrm{ab}^{-1}$ of data collected with $P\left(e^{-}, e^{+}\right)=(-0.8,+0.3)$ beam polarization would contain approximatively $6.4 \times 10^{5}$ Higgses. The latter polarization configuration maximizes the $e^{+} e^{-} \rightarrow h Z$ cross section. The recoil mass of the $Z$ gives access to the inclusive $e^{+} e^{-} \rightarrow h Z$ rate independently of the exclusive Higgs decay channels measurements. The Higgsstrahlung process can also be measured at higher center-of-mass energies. Despite the smaller cross sections, this allows to probe different combinations of EFT parameters and is thus helpful for resolving (approximate) degeneracies among them. The estimated measurement precisions at each collider and at different energies are shown in table 2, 3 and 4 of appendix B, where further details are also provided.

A few important comments are in order. As mentioned in section 2, the measurement of the rare $h \rightarrow Z \gamma$ decay, while not very constraining for the SM $h Z \gamma$ coupling, could be very important to resolve the degeneracies of EFT parameters in the production processes. Therefore, while the estimation of this measurement is not available for the FCC-ee and ILC, we scale the precision estimated for the CEPC, assuming the dominance of statistical uncertainties. Some care must also be taken to avoid potential double counting between the $e^{+} e^{-} \rightarrow h Z, Z \rightarrow \nu \bar{\nu}, h \rightarrow b \bar{b}$ process and the weak-boson fusion $e^{+} e^{-} \rightarrow \nu \bar{\nu} h, h \rightarrow b \bar{b}$, which yield the same final state. This is further discussed in section 3.2 and appendix B. Note also that the interferences between $s$-channel $Z$ and photon amplitudes are accidentally suppressed by a factor of $1-4 \sin ^{2} \theta_{W} \simeq 0.06$ in the total unpolarized cross section. This factor arises from the sum of the left- and right-handed couplings of the electron to the $Z, \frac{e}{2 s_{W} c_{W}}\left(-1+2 s_{W}^{2}\right)$ and $\frac{e}{2 s_{W} c_{W}}\left(2 s_{W}^{2}\right)$, respectively. Beam polarization thus significantly affects the sensitivity of the Higgsstrahlung rate to operators contributing to the $h Z \gamma$ vertex. ${ }^{4}$ Numerical expressions in the Higgs basis are provided in eq. (D.1). Introducing $c_{\gamma \square}$ defined in eq. (A.3) and contributing for an off-shell photon however renders this effect more transparent. For $P\left(e^{-}, e^{+}\right)=(0,0),(-0.8,+0.3),(+0.8,-0.3)$ polarization

\footnotetext{
${ }^{4}$ We thank Michael Peskin for helping us understand this interesting phenomena.
} 


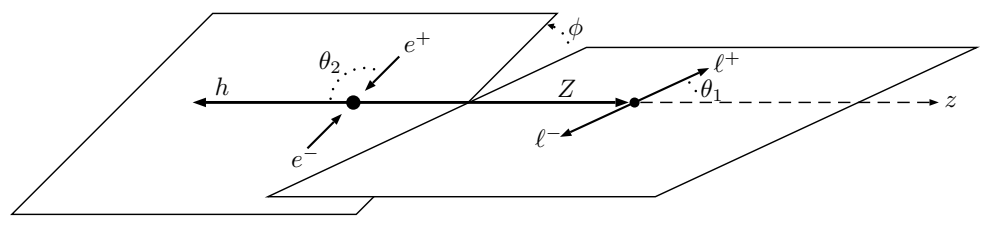

Figure 2. Definition of the $\Omega=\left\{\theta_{1}, \theta_{2}, \phi\right\}$ angles in a $e^{+} e^{-} \rightarrow h Z$ event (taken from ref. [31]). Note the two polar angles are respectively defined in the center-of-mass and $Z$ restframes.

configurations at $\sqrt{s}=250 \mathrm{GeV}$, we for instance obtain:

$$
\left.\frac{\sigma_{h Z}}{\sigma_{h Z}^{\mathrm{SM}}}\right|_{250 \mathrm{GeV}} ^{P=\left(\begin{array}{c}
(0,0) \\
(-0.8,+0.3) \\
(+0.8,-0.3)
\end{array}\right)} \simeq 1+2 \delta c_{Z}+1.6 c_{Z Z}+3.5 c_{Z \square}+\left(\begin{array}{c}
0.060 \\
0.82 \\
-0.89
\end{array}\right) c_{Z \gamma}+\left(\begin{array}{c}
0.16 \\
2.2 \\
-2.3
\end{array}\right) c_{\gamma \square} .
$$

An increase in the sensitivity magnitude of more than an order of magnitude is brought by beam polarization. Reversing the polarization also flips the sign of the $c_{Z \gamma}$ and $c_{\gamma} \square$ prefactors, given the opposite signs of the left- and right-handed couplings of the $Z$ to electrons.

Angular asymmetries. Three angles and two invariant masses fully characterize the differential distribution of the $e^{+} e^{-} \rightarrow h Z \rightarrow h f \bar{f}$ process (see figure 2). It naturally provides information complementary to that of the total rate alone. The effective-fieldtheory contributions to the angular distributions have been thoroughly studied in ref. [29]. At tree level and linear order in the effective-field-theory parameters, they can all be captured through the following asymmetries:

$$
\begin{aligned}
\mathcal{A}_{\theta_{1}} & =\frac{1}{\sigma} \int \mathrm{d} \Omega \operatorname{sgn}\left\{\cos \left(2 \theta_{1}\right)\right\} \frac{\mathrm{d} \sigma}{\mathrm{d} \Omega}, \\
\mathcal{A}_{\phi}^{(1)} & =\frac{1}{\sigma} \int \mathrm{d} \Omega \operatorname{sgn}\{\sin \phi\} \frac{\mathrm{d} \sigma}{\mathrm{d} \Omega}, \\
\mathcal{A}_{\phi}^{(2)} & =\frac{1}{\sigma} \int \mathrm{d} \Omega \operatorname{sgn}\{\sin (2 \phi)\} \frac{\mathrm{d} \sigma}{\mathrm{d} \Omega}, \\
\mathcal{A}_{\phi}^{(3)} & =\frac{1}{\sigma} \int \mathrm{d} \Omega \operatorname{sgn}\{\cos \phi\} \frac{\mathrm{d} \sigma}{\mathrm{d} \Omega}, \\
\mathcal{A}_{\phi}^{(4)} & =\frac{1}{\sigma} \int \mathrm{d} \Omega \operatorname{sgn}\{\cos (2 \phi)\} \frac{\mathrm{d} \sigma}{\mathrm{d} \Omega}, \\
\mathcal{A}_{c \theta_{1}, c \theta_{2}} & =\frac{1}{\sigma} \int \mathrm{d} \Omega \operatorname{sgn}\left\{\cos \theta_{1} \cos \theta_{2}\right\} \frac{\mathrm{d} \sigma}{\mathrm{d} \Omega},
\end{aligned}
$$

where $\Omega=\left\{\theta_{1}, \theta_{2}, \phi\right\}$ and the sgn function gives the sign of its argument. Among these asymmetries, $\mathcal{A}_{\phi}^{(1)}$ and $\mathcal{A}_{\phi}^{(2)}$ are sensitive to CP-violating parameters (or absorptive parts of amplitude), while $\mathcal{A}_{\theta_{1}}$ and $\mathcal{A}_{\phi}^{(4)}$ depend on the same combination of operator coefficients. In the absence of $\mathrm{CP}$ violation, the angular observables therefore provide three independent constraints on effective-field-theory parameters. The corresponding Higgs-basis expressions are provided in appendix D. 

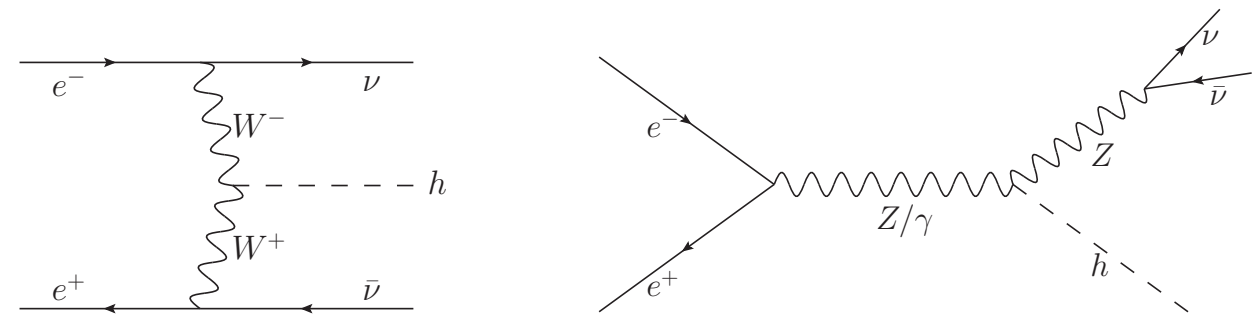

Figure 3. Two contributions to the $e^{+} e^{-} \rightarrow \nu \bar{\nu} h$ process: weak-boson fusion (left), and $e^{+} e^{-} \rightarrow$ $h Z, Z \rightarrow \nu \bar{\nu}$ (right).

A phenomenological study of these angular asymmetries at circular $e^{+} e^{-}$colliders has been performed in ref. [31]. In particular, it was shown that the uncertainties on their determination is statistics dominated for leptonic $Z$ decays. The absolute statistical uncertainty (one standard deviation) on each asymmetry $\mathcal{A}$ measured with $N$ events is given by [31]

$$
\sigma_{\mathcal{A}}=\sqrt{\frac{1-\mathcal{A}^{2}}{N}} \approx \frac{1}{\sqrt{N}}
$$

Following ref. [31], we use only the events with Higgs decays to bottom quarks $\left(e^{+} e^{-} \rightarrow\right.$ $\left.h Z, Z \rightarrow \ell^{+} \ell^{-}, h \rightarrow b \bar{b}\right)$ which has negligible backgrounds. Reference [31] refers to a preliminary version of the CEPC preCDR which suggests the signal selection efficiency of this channel at $240 \mathrm{GeV}$ is around $54 \%$. For simplicity, we assume a universal efficiency of $60 \%$ for the event selection of this channel at all energies for the angular asymmetry analysis. For the CEPC, with $5 \mathrm{ab}^{-1}$ collected at $240 \mathrm{GeV}$, this constitutes a subsample of approximately $2.7 \times 10^{4}$ Higgsstrahlung events. For the ILC, the effects of beam polarizations on the asymmetries is taken into account. No systematic uncertainty is included. We however expect statistical uncertainties to be dominant given the fairly rare but clean $Z$ decay to leptons.

\subsection{Higgs production through weak boson fusion}

The Higgs couplings to $W, Z$ bosons, and photons are related by $\mathrm{SU}(2)_{L}$ gauge invariance. As such, the measurement of the weak-boson fusion process, first considered in $e^{+} e^{-}$colliders in ref. [61], is complementary to that of the Higgsstrahlung process. So, a combination of the two measurements can efficiently resolve the degeneracy among the EFT parameters that contribute to the production processes. The weak-boson fusion cross section grows with energy, so that it is better measured at a center-of-mass energy of $350 \mathrm{GeV}$ or above. Nevertheless, the measurement at $240 \mathrm{GeV}$ can still provide important information, especially if runs at higher energies are not performed.

Importantly, Higgsstrahlung with $Z$ decay to neutrinos $\left(e^{+} e^{-} \rightarrow h Z, Z \rightarrow \nu \bar{\nu}\right)$ yields the same final state as weak-boson fusion (see figure 3 ) and has a rate about six times larger at a center-of-mass energy of $240 \mathrm{GeV}$ (without beam polarization). At this center-of-mass energy the missing mass distributions for both processes moreover peak at similar energies (see figure 3.16 on page 75 of ref. [1]). Isolating the weak-boson fusion contribution is therefore difficult. For the CEPC and FCC-ee at $240 \mathrm{GeV}$, we therefore consider an inclusive 

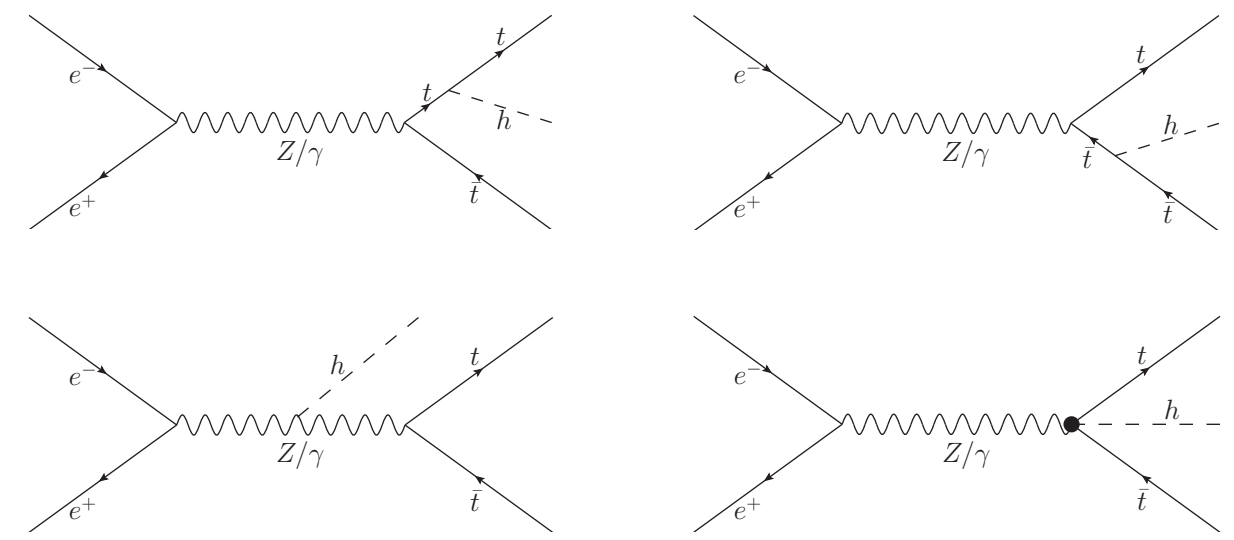

Figure 4. Leading-order diagrams for the $e^{+} e^{-} \rightarrow t \bar{t} h$ process. In the SM, the dominant contribution are the ones involving the top Yukawa coupling. Other EFT contributions (including that of four-fermion operators, not depicted) should be well constrained by other measurements.

$e^{+} e^{-} \rightarrow \nu \bar{\nu} h$ sample to which the two processes contribute, and only use the $h \rightarrow b \bar{b}$ channel for which the precision on the $e^{+} e^{-} \rightarrow \nu \bar{\nu} h$ rate measurement is reported in the literature. We neglect the contributions of the weak-boson fusion in the other Higgs decay channels of $e^{+} e^{-} \rightarrow h Z, Z \rightarrow \nu \bar{\nu}$. For the ILC, ref. [59] states that a $\chi^{2}$ fit of the recoil mass distribution is used to separate the weak-boson-fusion and the Higgsstrahlung processes. We thus consider that the precision on $\sigma\left(e^{+} e^{-} \rightarrow \nu \bar{\nu} h\right) \times \mathrm{BR}(h \rightarrow b \bar{b})$ quoted in ref. [58] applies directly to the weak-boson fusion contribution. Both processes reach equal rates at a centerof-mass energy close to $350 \mathrm{GeV}$ (without beam polarization). At this and higher energies, we thus assume that their distinct recoil-mass distributions are sufficient to efficiently separate them. More details on the treatment of this measurement can be found in appendix B.

\subsection{Higgs production in association with tops}

The $e^{+} e^{-} \rightarrow t \bar{t} h$ production of a Higgs boson in association with top quarks (see figure 4) requires a large center-of-mass energy which is only achieved at a linear collider. A $10 \%$ precision on $\sigma(t \bar{t} h) \times \mathrm{BR}(h \rightarrow b \bar{b})$ could be achieved with $4 \mathrm{ab}^{-1}$ of ILC data collected at $\sqrt{s}=500 \mathrm{GeV}$ (scaled from $28 \%$ of the $500 \mathrm{fb}^{-1}$ result in ref. [58]). At CLIC, $1.5 \mathrm{ab}^{-1}$ of $1.4 \mathrm{TeV}$ data should yield an $8.4 \%$ precision [60]. In the SM, the dominant contributions to this process involve a top Yukawa coupling. The radiation of a Higgs from the $s$-channel $Z$ boson is comparatively negligible [3]. In the effective field theory, we only include modifications of the top Yukawa coupling. Other contributions should be sufficiently constrained by the measurement of top pair production and other processes. Neither the four-point $Z h t t$ interaction depicted on figure 4 (bottom-right), nor four-fermion operator contributions are thus accounted for here. This channel could also be used to establish the CP properties of the Higgs boson [62], which we simply assumed to be a $0^{+}$state throughout our analysis.

\subsection{Weak-boson pair production}

The diagrams contributing to the $e^{+} e^{-} \rightarrow W W$ process, at leading order, are depicted in figure 5 . The $s$-channel diagrams with an intermediate $Z$ or photon involve triple gauge 

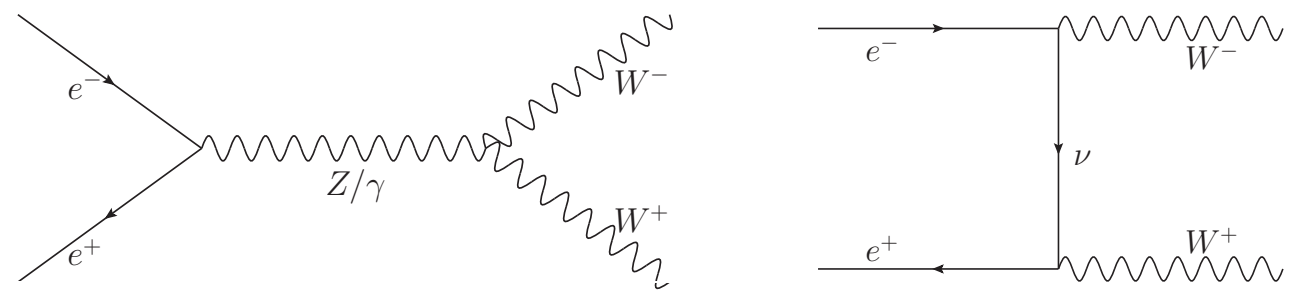

Figure 5. Leading-order diagrams contributing to $e^{+} e^{-} \rightarrow W W$. The $s$-channel diagram on the left with an intermediate $Z$ or photon involves a triple gauge coupling.

couplings. Considering CP-even dimension-six operators only, the aTGCs are traditionally parameterized using $\delta g_{1, Z}, \delta \kappa_{\gamma}$ and $\lambda_{Z}$ [63, 64], defined in eq. (A.5). Among them, $\delta g_{1, Z}$ and $\delta \kappa_{\gamma}$ are generated by effective operators that also contribute to Higgs observables. As pointed out in ref. [23], this leads to an interesting interplay between Higgs and TGC measurements.

Triple gauge couplings have been measured thoroughly at LEP2 [65]. Various studies of future lepton colliders' reach have also been carried out [66-71]. At future circular colliders, most of the $W$ pairs are likely to be produced at $240 \mathrm{GeV}$, as a byproduct of the Higgs measurement run which requires large luminosities. At this energy, the $e^{+} e^{-} \rightarrow$ $W W$ cross section is approximately two orders of magnitude larger than that of $e^{+} e^{-} \rightarrow$ $h Z$. With $5 \mathrm{ab}^{-1}$, the CEPC would thus produce about $9 \times 10^{7} e^{+} e^{-} \rightarrow W W$ events, thereby improving significantly our knowledge of TGCs. A run at $350 \mathrm{GeV}$, probing a different combination, could bring further improvement on the constraints. Longitudinal beam polarization is also very helpful in probing the aTGCs. With $500 \mathrm{fb}^{-1}$ collected at $500 \mathrm{GeV}$ and equally shared between four $P\left(e^{-}, e^{+}\right)=( \pm 80 \%, \pm 30 \%)$ beam polarization configurations, the ILC could constrain the three TGCs to the $10^{-4}$ level [68]. Note the runs with ++ and -- polarizations are mostly meant to provide a simultaneous and sufficiently accurate polarization magnitude measurement. Comparable results can be expected for more realistic repartitions of the luminosities [69].

For the CEPC and FCC-ee prospects, we follow ref. [71] which exploited kinematic distributions in the $e^{+} e^{-} \rightarrow W W \rightarrow 4 f$ process. Five angles can be reconstructed in each such event: the polar angle between the incoming $e^{-}$and the outgoing $W^{-}$, and two angles specifying the kinematics of each $W$ decay products. When both $W$ s decay leptonically, the $W$ mass constraints allow to fully reconstruct the kinematics up to a fourfold ambiguity at most. Here, we make the optimistic assumption that the correct solution is always found. In the hadronic $W$ decays, one can not discriminate between the quark and antiquark. The angular distributions of the $W$ decay products are thus folded. We divide the differential distributions of each angle into 20 bins (10 in folded distributions). Uncorrelated Poisson distributions are assumed in each bin and their $\chi^{2}$ are summed over. The total $\chi^{2}$ is constructed by summing over the $\chi^{2}$ of all the angular distributions of all decay channels. The statistical correlation between angular distributions is neglected.

Given the huge statistics that would be collected, and although they were neglected in ref. [71], the systematic uncertainties could play an important role. Theoretical uncertain- 
ties could also become limiting. At the moment, there is however no dedicated experimental study of TGC measurements at future circular colliders. We therefore introduce a benchmark systematic uncertainty of $1 \%$ in each bin of the differential distributions. This guess is probably too conservative compared to few $10^{-4}$ systematic uncertainties on the $\delta g_{1, Z}$, $\delta \kappa_{\gamma}$, and $\lambda_{Z}$ TGC parameters recently estimated by the ILC collaboration [72]. We therefore examine the impact of variation of this value in section 4 and also provide constraints obtained by assuming no deviation on the TGC from their standard-model values.

For the prospects of the full ILC program, we use the one-sigma statistical uncertainties obtained in ref. [68] $\left(\Delta \delta g_{1, Z}=6.1 \times 10^{-4}, \Delta \delta \kappa_{\gamma}=6.4 \times 10^{-4}\right.$ and $\left.\Delta \lambda_{Z}=7.2 \times 10^{-4}\right)$, together with their correlations shown in table 7 of appendix B. We do however not scale these numbers to higher luminosities, as systematic uncertainties are likely to become important. The current estimates by the ILC collaboration for systematics uncertainties are of a few $10^{-4}[72]$. When focusing on the 250 and $350 \mathrm{GeV}$ runs of the ILC, we use the strategy described above for the CEPC instead. As a dedicated experimental study of TGC measurements at CLIC is also missing, ${ }^{5}$ we assume a precision similar to the ILC one can be reached there. It should be noted, however, that the 1.4 and $3 \mathrm{TeV}$ runs at CLIC could potentially provide even stronger constraints on the aTGCs due to the increase of sensitivities with energy [35].

Another important issue raised by the significant improvement in the $e^{+} e^{-} \rightarrow W W \rightarrow$ if measurement precision concerns the uncertainty on electroweak precision observables. In the extraction of the constraints on aTGCs, one usually makes the TGC dominance assumption and neglects the impact of new physics on all other parameters. At LEP, this was justified given the better precision of $Z$-pole and $W$-mass measurements compared to that of $W$ pair production. In this work, we also assume that runs at lower energies will give us sufficient control on such effects. Exploiting diboson data could also be an alternative if runs at lower energies are not performed. Further investigations are required in this direction. The $W$ mass can be measured very well at a Higgs factory by reconstructing the $W$ decay products in the $e^{+} e^{-} \rightarrow W W$ process. To leading order, the aTGCs affect the differential distributions of $e^{+} e^{-} \rightarrow W W$, but not the $W$ invariant mass. The two measurements are thus approximatively independent. A precision of $3 \mathrm{MeV}$ could be achieved at the CEPC with this method [1]. A dedicated threshold scan at center-of-mass energies of $160-170 \mathrm{GeV}$ could also be performed. As such, it is reasonable to assume the $W$ mass will be sufficiently well constrained at future $e^{+} e^{-}$colliders. The corrections to gauge-boson propagators and fermion gauge couplings could however have a non-negligible impact on the determination of triple gauge couplings, especially without a future $Z$ factory to improve their constraints. ${ }^{6}$ While the CEPC and FCC-ee could perform a run at

\footnotetext{
${ }^{5}$ For CLIC at $3 \mathrm{TeV}$ and an integrated luminosity of $1 \mathrm{ab}^{-1}$, ref. [67] bases itself on ref. [66] which derived individual constraints and quotes $\Delta \delta \kappa_{\gamma}=0.9 \times 10^{-4}, \Delta \lambda_{Z}=1.3 \times 10^{-4}$ constraints (we thank Philipp Roloff for pointing out this reference). These results are however insufficient to serve as input for our global analysis. A phenomenological study for CLIC based on total $e^{+} e^{-} \rightarrow W W$ rates only was also performed in ref. [35]. The results in section 3.2 and eq. (4.2) there imply individual constraints rescaled for $1 \mathrm{ab}^{-1}$ that are less than a factor of two better than that of ref. [67].

${ }^{6}$ See also ref. [25] for a recent discussion on this topic in the context of LHC measurements.
} 
the $Z$ pole, the interest of such a $Z$-pole run at the ILC and CLIC is still under investigation. Notably, the ILC precision on aTGCs quoted above already surpasses the precision obtained at LEP on the electroweak observables. A global fit including Higgs, TGC and the $Z$-pole measurements would be instructive but is beyond the scope of this paper.

\subsection{Global fit and determinant parameter}

Our total $\chi^{2}$ can be rewritten as the sum of that of the measurements described previously in this section:

$$
\chi_{\text {tot }}^{2}=\chi_{h Z / \nu \bar{\nu} h, \text { rates }}^{2}+\chi_{h Z, \text { asymmetries }}^{2}+\chi_{W W}^{2},
$$

where $^{7}$

$$
\begin{aligned}
\chi_{h Z / \nu \bar{\nu} h, \text { rates }}^{2} & =\sum_{i} \frac{\left(\mu_{i}^{\mathrm{NP}}-\mu_{i}^{\mathrm{SM}}\right)^{2}}{\sigma_{\mu_{i}}^{2}}, \\
\chi_{h Z, \text { asymmetries }}^{2} & =\sum_{i} \frac{\left(\mathcal{A}_{i}^{\mathrm{NP}}-\mathcal{A}_{i}^{\mathrm{SM}}\right)^{2}}{\sigma_{\mathcal{A}_{i}}^{2}}, \\
\chi_{W W \text { (CEPC \& FCC-ee) }}^{2} & =\sum_{i} \frac{\left(n_{i}^{\mathrm{NP}}-n_{i}^{\mathrm{SM}}\right)^{2}}{\left(\sqrt{n_{i}}+\sigma_{i}^{\mathrm{sys}}\right)^{2}} .
\end{aligned}
$$

The $\mu_{i}$ are the signal strengths (rates normalized to SM predictions) of the rate measurements, summed over $\sigma(h Z), \sigma(h Z) \times \mathrm{BR}$ and $\sigma(\nu \bar{\nu} h) \times \mathrm{BR}$. The corresponding one-sigma uncertainties are listed in table 2,3 and 4 of appendix B, for the different colliders. $\mathcal{A}_{i}$ are the asymmetries of eq. (3.2), and $\sigma_{\mathcal{A}_{i}}$ their uncertainties, given in eq. (3.3). For the $e^{+} e^{-} \rightarrow W W$ measurements at CEPC and FCC-ee, the $\chi^{2}$ is summed over all $W$-boson decay channels, over the five angular distributions, and over all their bins. A systematic uncertainty $\sigma_{i}^{\text {sys }}$ is included in each bin. Unless otherwise specified, we take $\sigma_{i}^{\text {sys }} / n_{i}=1 \%$ where $n_{i}$ is the number of events in that bin. For ILC and CLIC, the $\chi_{W W}^{2}$ is directly reconstructed from one-sigma bounds and the correlation matrix of aTGCs from ref. [68] (shown in table 7 of appendix B). Finally, the $\chi^{2}$ is summed over runs with different energies and beam polarizations (if applicable).

As we only retained the linear dependence of all observables in terms of effectiveoperator coefficients, our $\chi^{2}$ are quadratic functions:

$$
\chi^{2}=\sum_{i j}\left(c-c_{0}\right)_{i} \sigma_{i j}^{-2}\left(c-c_{0}\right)_{j}, \quad \text { where } \quad \sigma_{i j}^{-2} \equiv\left(\delta c_{i} \rho_{i j} \delta c_{j}\right)^{-1},
$$

where $c_{i=1, \ldots 12}$ denotes the 12 parameters of eq. (2.1) and $c_{0}$ are the corresponding central values, which are vanishing by construction in our study. The uncertainties $\delta c_{i}$ and the correlation matrix $\rho$ can thus be obtained from $\sigma_{i j}^{-2}=\partial^{2} \chi^{2} / \partial c_{i} \partial c_{j}$.

It should also be noted that the measured Higgs decay width reported in the corresponding documents of the colliders is a quantity derived (with certain assumptions) from several measurements which are already included in the fit. We therefore do not include it in our fit as an additional independent measurement. 


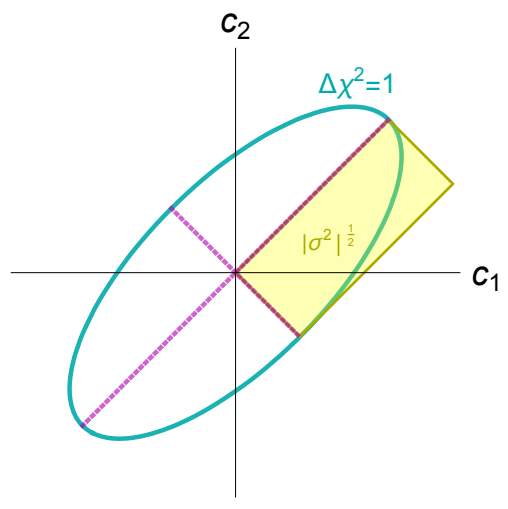

Figure 6. In a two-dimensional parameter space, the area of the Gaussian one-sigma ellipse is proportional to the square root of the determinant of the covariance matrix, $\sqrt{\operatorname{det} \sigma^{2}}$. In $n$ dimensions, the $n$th root of this quantity or global determinant parameter (GDP $\equiv \sqrt[2 n]{\operatorname{det} \sigma^{2}}$ ) provides an average of constraints strengths. GDP ratios measure improvements in global constraint strengths independently of an effective-field-theory operator basis.

Global determinant parameter (GDP). We introduce a metric, dubbed global determinant parameter, for assessing the overall strength of constraints. In a global analysis featuring $n$ degrees of freedom, it is defined as the determinant of the covariance matrix raised to the $1 / 2 n$ power, GDP $\equiv \sqrt[2 n]{\operatorname{det} \sigma^{2}}$. In a multivariate Gaussian problem, the square root of the determinant is proportional to the volume of the one-sigma ellipsoid $\left(\pi^{\frac{n}{2}} / \Gamma\left(\frac{n}{2}+1\right) \sqrt{\operatorname{det} \sigma^{2}}\right)$ and therefore measures the allowed parameter space size (see figure 6). Its $n$th root is the geometric average of the half lengths of the ellipsoid axes and can thus serve as an average constraint strength. Interestingly, the ellipsoid volume transforms linearly under rescalings of the fit parameters. So, ratios of GDPs do not depend on parameters' normalization. They are obviously also invariant under rotations in the multidimensional parameters space. Such ratios are thus independent on the choice of effective-operator basis used to describe the same underlying physics. We therefore judge these quantities especially convenient to measure the improvement in global constraints brought by different run scenarios of future lepton colliders. It is however to be noted that the GDP measure weights equally all directions in the effective-field-theory parameter space, so that it is on its own certainly not accounting for the fact some directions are privileged by specific power countings or models.

\section{Results}

We first discuss in this section the precision reach of the whole program of each collider before examining, in subsequent subsections, the impact of different measurements, centerof-mass energies, systematic uncertainties, and beam polarization. The CEPC is then taken as an illustrative example (except when studying polarization) and the corresponding figures for the FCC-ee and ILC are provided in appendix C.

\footnotetext{
${ }^{7}$ Note that we have used the symbol $\sigma$ to denote both cross sections and standard deviations. What we mean in each case should be clear from the context.
} 


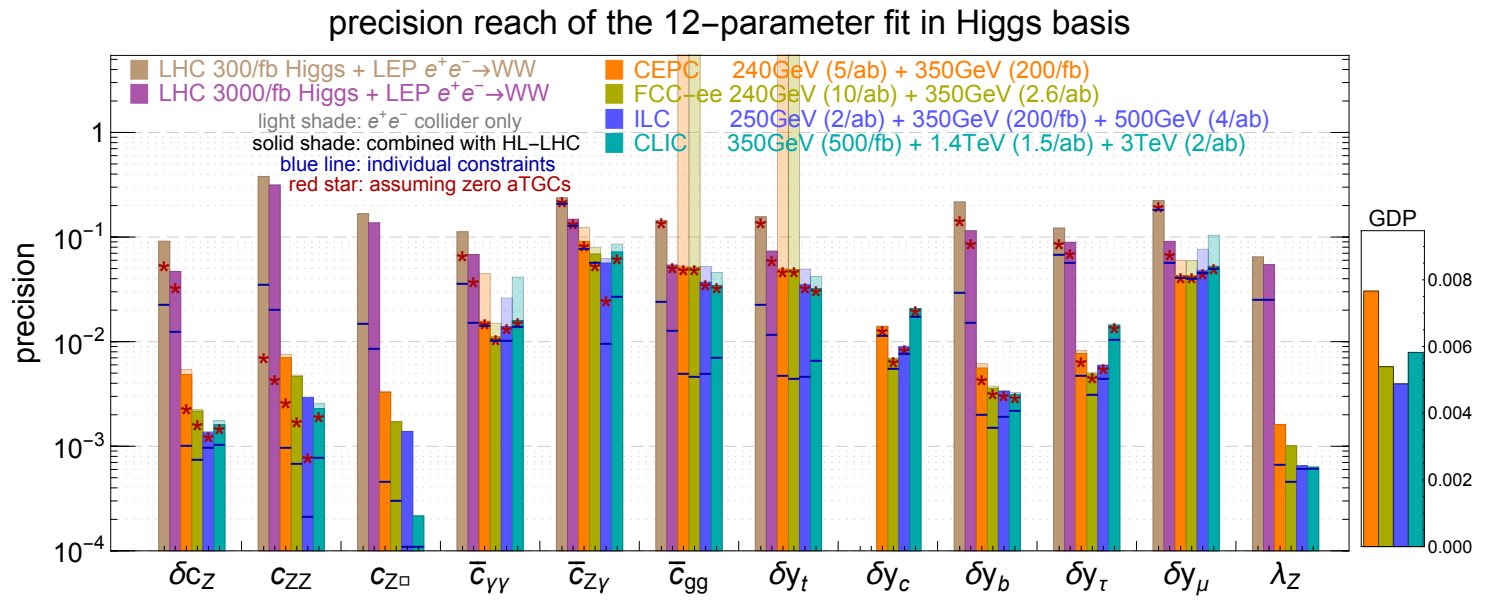

Figure 7. One-sigma precision reach of future lepton colliders on our effective-field-theory parameters. All results but the light-shaded columns include the $14 \mathrm{TeV}$ LHC (with $3000 \mathrm{fb}^{-1}$ ) and LEP measurements. LHC constraints also include measurements carried out at $8 \mathrm{TeV}$. Note that, without run above the $t \bar{t} h$ threshold, circular colliders alone do not constrain the $\bar{c}_{g g}$ and $\delta y_{t}$ effective-fieldtheory parameter individually. The combination with LHC measurements however resolves this flat direction. The horizontal blue lines on each column correspond to the constraints obtained when one single parameter is kept at the time, assuming all others vanish. The red stars correspond to the constraints assuming vanishing aTGCs. The GDPs of future lepton colliders are shown on the right panel. See main text for comparisons with the LHC GDPs.

We show in figure 7 the one-sigma precision reach at various future lepton colliders on our effective-field-theory parameters. These projections are compared to the reach of the Higgs measurements at the $14 \mathrm{TeV}$ LHC with $300 \mathrm{fb}^{-1}$ and $3000 \mathrm{fb}^{-1}$ of integrated luminosity, combined with the diboson production measurement at LEP. The estimated reach of Higgs measurements at the high-luminosity LHC derives from projection by the ATLAS collaboration [73] which collected information from various other sources. Information about the composition of each channel are extracted from refs. [74-78]. Theory uncertainties on these LHC measurements are not included in our estimations. In LHC results, we also assume the charm Yukawa to be SM-like as ref. [73] does not provide estimations on the $h \rightarrow c \bar{c}$ branching fraction precision reach. The constraints from the diboson measurements at LEP are obtained from ref. [23]. We do not include the LHC constraints arising from diboson production, as issues related to the validity of the effective-field-theory $[24,79]$ and of the TGC dominance assumption [25] need to be simultaneously considered. A dedicated study of the reach of the high-luminosity LHC on these processes should be carried out. The constraints set at future lepton colliders are however expected to be much more stringent.

Compared with LHC and LEP, future lepton colliders would improve the measurements of effective-field-theory parameters by roughly one order of magnitude. A combination with the LHC measurements provides a marginal improvement for most of the parameters. For $\bar{c}_{\gamma \gamma}, \bar{c}_{Z \gamma}$ and $\delta y_{\mu}$, the improvements are more significant, as the small rates and clean signals make the LHC reaches comparable to that of lepton colliders. It should be noted that the measurements of the $h \rightarrow g g$ branching fraction only constrain a linear combination of $\bar{c}_{g g}$ 
and $\delta y_{t}$. These two parameters are thus only constrained independently by lepton colliders when $t \bar{t} h$ production is measured. Therefore, the combination with LHC measurements is required for CEPC and FCC-ee to constrain $\bar{c}_{g g}$ and $\delta y_{t}$. The resulting bounds on $\delta y_{t}$ are then even substantially better than that set by the LHC alone.

The twelve-parameter GDPs for the combination of future lepton collider, LHC $3000 \mathrm{fb}^{-1}$ and LEP measurements are displayed on the right panel of figure 7 . Corresponding numerical values are 0.0077, 0.0054, 0.0049, 0.0058 for CEPC, FCC-ee, ILC and CLIC, respectively. Varying prospective constraints on the charm Yukawa measurement complicate the comparison with the high-luminosity LHC. The ATLAS collaboration estimated the $h \rightarrow J / \psi \gamma$ branching fraction could be constrained to be smaller than 15 times its standard model value with $3 \mathrm{ab}^{-1}$ at $14 \mathrm{TeV}$ [80]. Such a constraint would translate into a one-sigma precision reach on $\delta y_{c}$ of order one. To broadly cover the range spent by other studies [81-85], we vary the expected precision reach on $\delta y_{c}$ in the $0.01-10$ range. The combination of LHC $300 \mathrm{fb}^{-1}\left(3000 \mathrm{fb}^{-1}\right)$ and LEP measurements only then leads to GDPs in the $0.065-0.116(0.039-0.069)$ interval, one order of magnitude worst than when future lepton collider measurements are included. On the other hand, with $\delta y_{c}$ set to zero, the eleven-parameter GDP for the combination of LHC $300 \mathrm{fb}^{-1}\left(3000 \mathrm{fb}^{-1}\right)$ and LEP measurements only is of 0.078 (0.044). In comparison, when future lepton collider measurements are also included, the corresponding eleven-parameter GDP are 0.0073, 0.0053, 0.0046, 0.0052 for CEPC, FCC-ee, ILC and CLIC, respectively.

Let us also comment further on the impact of having discarded the quadratic dependence on dimension-six operator coefficients. As stressed in section 2, no significant effect is expected given the good precision achieved at future lepton colliders in the measurement of most observable. Note that even the branching ratios for rare Higgs decays like $h \rightarrow \gamma Z$ are sufficiently well constrained for quadratic contributions to be subleading. Only cases in which accidental suppressions of the standard-model interference with effective-field-theory amplitudes require a case-by-case discussion. We identify two such cases. First, helicity selection rules are known to suppress the ratio of linear and quadratic dependences on the $\lambda_{Z}$ aTGC at high energies. Reproducing the analysis made at $250 \mathrm{GeV}$ for a center-of-mass energy of $500 \mathrm{GeV}$ and $500 \mathrm{fb}^{-1}$ shared between two beam polarization configurations, with and without quadratic aTGC contributions, we obtained differences in the derived limits of $10 \%$ at most. The linear approximation thus seems to be reasonably accurate in that case and no strong quadratic aTGC dependence should affect the bounds derived in ref. [68]. We also checked that quadratic contributions would be subleading at $\sqrt{s}=3 \mathrm{TeV}$, provided the whole differential information is included. The non-interference between standard-model and dimension-six operator indeed does not hold when the azimuthal angles of the $W$ decay products are not integrated over. Secondly, as noted in section 3.1, the interference between the $s$-channel photon and $Z$ amplitudes in the unpolarized Higgsstrahlung cross section suffers from an accidental numerical suppression. Moreover, at high energies, the Higgsstrahlung cross section goes down and so does the accuracy with which it can be measured. Therefore, one can expect the quadratic dependence on the operator modifying the $H Z \gamma$ vertex with an off-shell photon to be important in that specific case. Although we use unpolarized cross section measurements to determine CLIC reach on effective-field-theory 


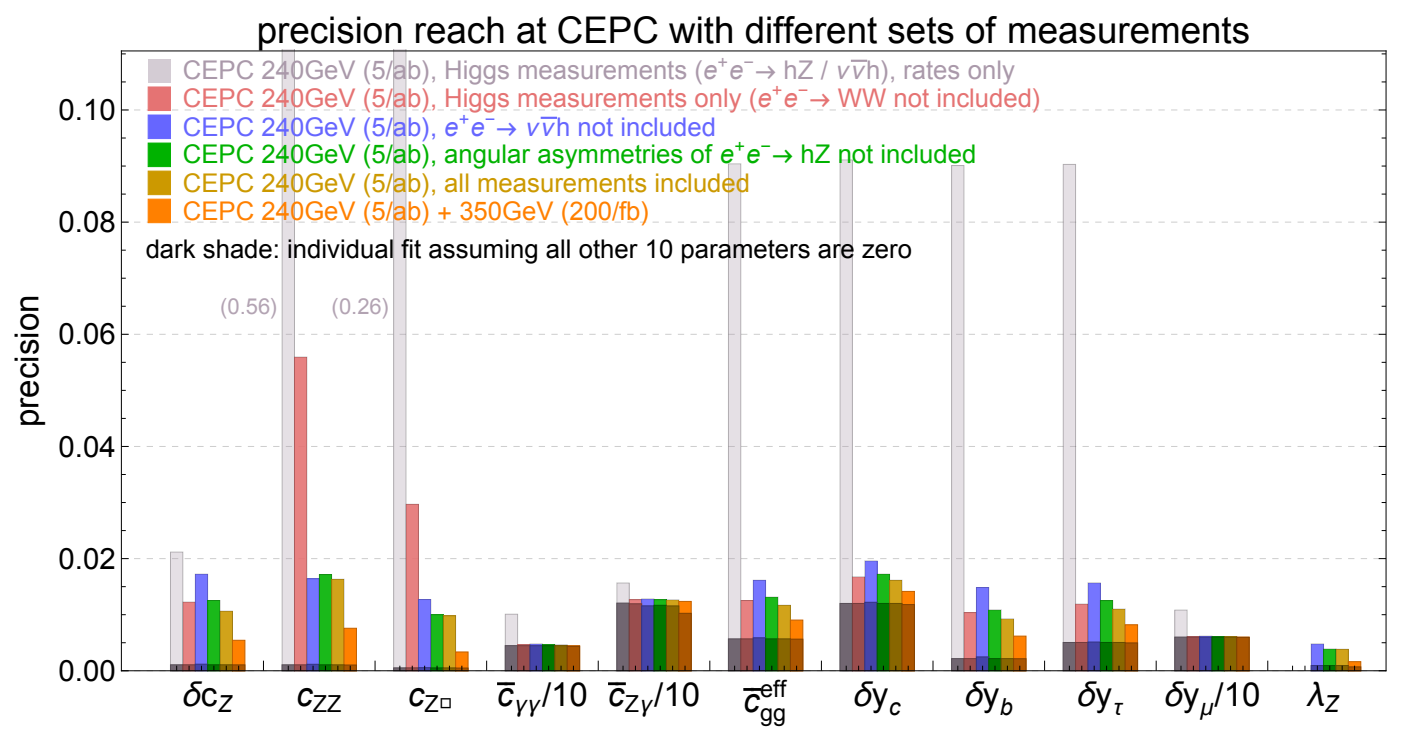

Figure 8. One-sigma precision reach obtained with various combinations of measurements at the CEPC. The first five columns exploit $5 \mathrm{ab}^{-1}$ of $240 \mathrm{GeV}$ data while the last column also includes $200 \mathrm{fb}^{-1}$ at $350 \mathrm{GeV}$. Only Higgs rate measurements $\left(e^{+} e^{-} \rightarrow h Z / \nu \bar{\nu} h\right)$ are included in the first column. One single measurement is excluded at the time in the three subsequent columns: $e^{+} e^{-} \rightarrow$ $W W$ in the second, $e^{+} e^{-} \rightarrow \nu \bar{\nu} h$ in the third, and the angular asymmetries of $e^{+} e^{-} \rightarrow h Z$ in the fourth. Note that $\lambda_{Z}$ is left unconstrained by Higgs data. All measurements at $240 \mathrm{GeV}$ are included to obtain the constraints in the fifth column. A run at $350 \mathrm{GeV}$ is also included in the last, sixth, column. The dark shades correspond to the constraints obtained when one single parameter is kept at the time, assuming all other vanish.

parameters to match experimental studies, beam polarization would actually be available at CLIC and we checked explicitly that the quadratic effective-field-theory contributions become unimportant once measurements with polarized beams are performed.

Impact of the various measurements. We examine, in figure 8 , the impact of different measurements. The one-sigma precision are displayed with one or more measurements removed from the global fit, using CEPC as an example. Since the degeneracy between $\bar{c}_{g g}$ and $\delta y_{t}$ can not be resolved with measurements at 240 and $350 \mathrm{GeV}$, we display the constraint on $\bar{c}_{g g}^{\text {eff }}$, defined in eq. (2.3). The first five columns use the measurements at $240 \mathrm{GeV}$ $\left(5 \mathrm{ab}^{-1}\right)$ only. The first column on the left shows the results from rate measurements in Higgs processes $\left(e^{+} e^{-} \rightarrow h Z / \nu \bar{\nu} h\right)$ only. To obtain the second, third, and fourth columns, one single measurement is excluded at the time: $e^{+} e^{-} \rightarrow W W(2 \mathrm{nd}), e^{+} e^{-} \rightarrow \nu \bar{\nu} h$ (3rd), and the angular asymmetries of $e^{+} e^{-} \rightarrow h Z$ (4th), respectively. The fifth column expresses the constraints deriving from all measurements at $240 \mathrm{GeV}$. In the last column, $200 \mathrm{fb}^{-1}$ of data at $350 \mathrm{GeV}$ is also included. The dark shades finally display the constraints deriving when one single effective-field-theory parameter is kept at a time.

Figure 8 transparently demonstrates that the Higgs rate measurements alone are insufficient to constrain simultaneously all parameters to a satisfactory degree. They leave poorly constrained some directions of the multidimensional parameter space, thereby weak- 
ening the whole fit. As already stressed, in such a global treatment, the combination of several observables is capital to effectively bound all parameter combinations. The global strength of constraints is dramatically improved by the first few measurement which resolve approximate degeneracies. Once a sufficient number of constraints is imposed, the exclusion of one single observable does not dramatically affect the overall precision. The individual constraints (obtained by switching on one parameter at a time), on the other hand, receive little improvement from the additional measurements - a clear demonstration that global constraints are driven by approximate degeneracies. A marginal improvement of the constraints obtained for a given run would be obtained by including a set of observables even more complete than the one we use.

Impact of a $350 \mathrm{GeV}$ run at circular colliders. As already visible in figure 8, a $350 \mathrm{GeV}$ run significantly improves the strength of the constraints set by circular colliders. An important question for their design is the optimal amount of luminosity to gather at that energy, in view of the physics performance and the budget cost. In addition to the top mass and electroweak coupling measurements, the improvement on the precision of Higgs coupling could be considered too. This is addressed in figure 9 which shows the reach of the CEPC for increasing amounts of integrated luminosity collected at $350 \mathrm{GeV}$, from 0 to $2 \mathrm{ab}^{-1}$. It is clear that a run at this energy is able to lift further approximate degeneracy among effective-field-theory parameters. A GDP reduction of about $17 \%$ is obtained with only $200 \mathrm{fb}^{-1}$, and reaches about $34 \%$ with $2 \mathrm{ab}^{-1}$. The improvements on the $\bar{c}_{\gamma \gamma}, \bar{c}_{Z \gamma}$, and $\delta y_{\mu}$ effective parameters are less significant. The Higgs decay channels which provide the dominant constraints on these parameters suffer from small rates. These constraints are thus mainly statistics limited and approximate degeneracies play a secondary role. It should be noted that the estimations for Higgs measurements at $350 \mathrm{GeV}$ for various luminosities are obtained by scaling from the ones in table 2, assuming statistical uncertainties dominate. This assumption may cease to be valid for large integrated luminosities.

Impact of beam polarization at linear colliders. The possibility of longitudinal beam polarization constitutes a distinct advantage for linear colliders. Implementing it at circular colliders may be difficult (especially at high center-of-mass energies) and not economically feasible [2]. Dividing the total luminosity into multiple runs of different polarization configurations effectively provides several independent observables and helps constraining different direction of the effective-theory parameter space. In figure 10, we examine what subdivision of the total ILC luminosity at $250 \mathrm{GeV}$ would optimize the final precision reach. We follow the ILC TDR [3] and assume that the ILC could achieve a maximum beam polarization of $80 \%$ for electrons and $30 \%$ for positrons. Ref. [58] proposes a run plan with four polarization configurations $\operatorname{sgn}\left\{P\left(e^{-}, e^{+}\right)\right\}=(-,+),(+,-),(-,-),(+,+)$ and corresponding luminosity fractions of $67.5 \%, 22.5 \%, 5 \%$, and $5 \%$, respectively. The $(-,-)$ and $(+,+)$ polarizations could serve to probe exotic new physics, like electron dipole or Yukawa operators. They however suppress the rate of Higgs and gauge boson production and are thus not very helpful for the precision study of these processes. For simplicity, we will thus only consider the $(-,+)$ and $(+,-)$ polarizations. Uncertainty estimates are often only provided for an entire run in the $P\left(e^{-}, e^{+}\right)=(-0.8,+0.3)$ configuration. Scaling 


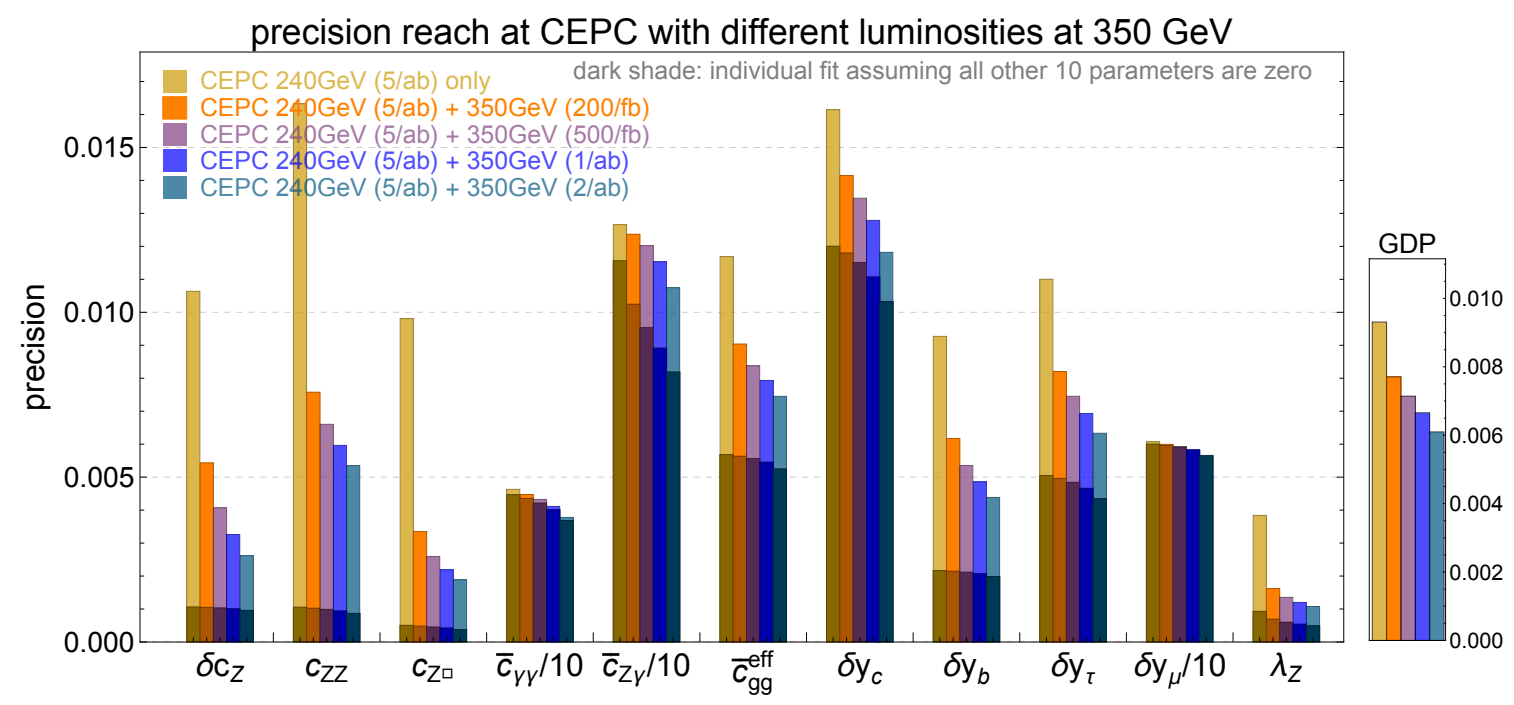

Figure 9. Precision reach (one standard deviation) of the 11-parameter fit in the Higgs basis at CEPC. Five different assumptions on the luminosity at $350 \mathrm{GeV}$ are made, namely 0 ( $240 \mathrm{GeV}$ run only), $200 \mathrm{fb}^{-1}, 500 \mathrm{fb}^{-1}, 1 \mathrm{ab}^{-1}$ and $2 \mathrm{ab}^{-1}$ (from left to right). The luminosity at $240 \mathrm{GeV}$ is fixed to $5 \mathrm{ab}^{-1}$. The parameters $\bar{c}_{\gamma \gamma}, \bar{c}_{Z \gamma}$ and $\bar{c}_{g g}^{\text {eff }}$ are defined in eq. (2.2) and eq. (2.3). The dark shades correspond to the constraints obtained when one single parameter is kept at the time, assuming all others vanish.

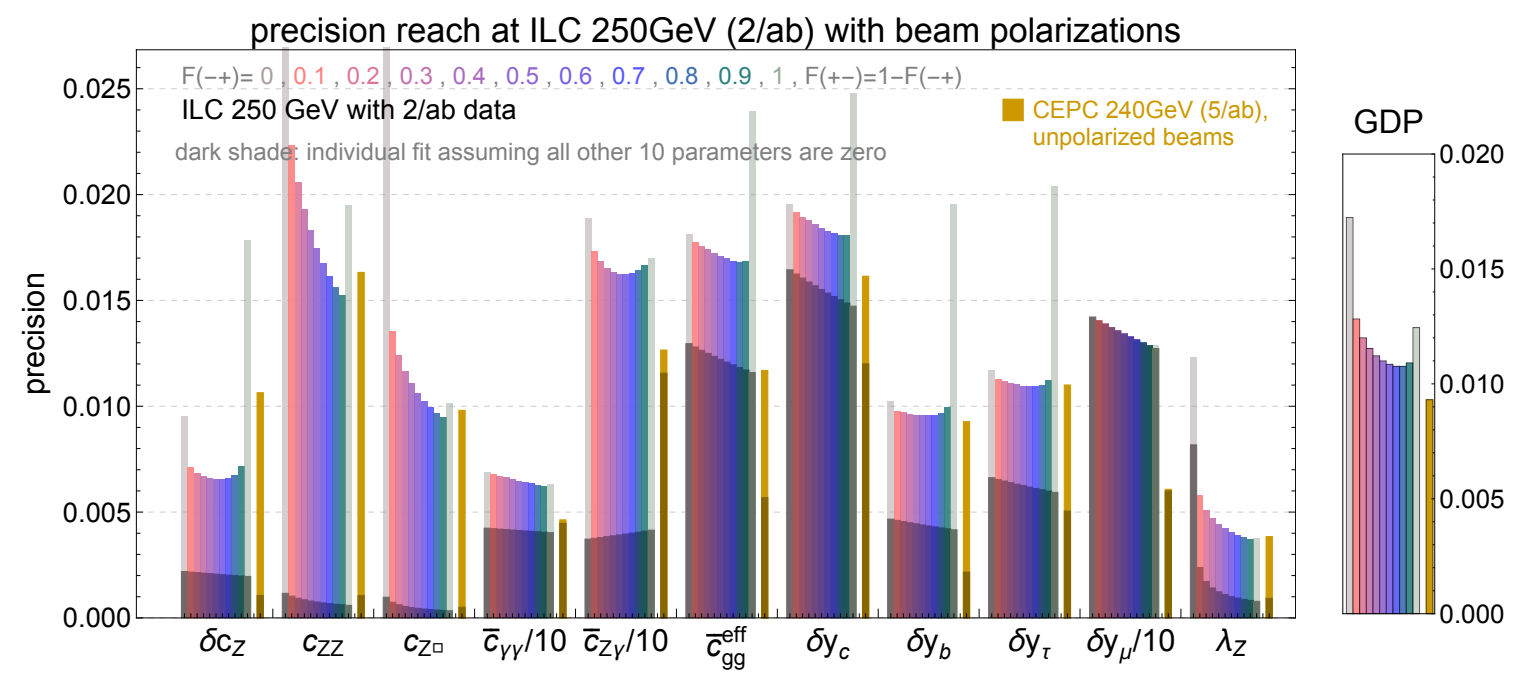

Figure 10. One-sigma precision reach of ILC runs at $250 \mathrm{GeV}$ with $2 \mathrm{ab}^{-1}$ of integrated luminosity shared between $P\left(e^{-}, e^{+}\right)=(-0.8,+0.3)$ and $(+0.8,-0.3)$ beam polarization configurations. The corresponding fractions are denoted as $F_{(-+)}$and $F_{(+-)}=1-F_{(-+)}$. For the sake of comparison, the constraints resulting from a CEPC run at $240 \mathrm{GeV}$ with $5 \mathrm{ab}^{-1}$ of integrated luminosity collected without beam polarization are also shown. The dark shades correspond to the constraints obtained when one single parameter is kept at the time, assuming all others vanish. 


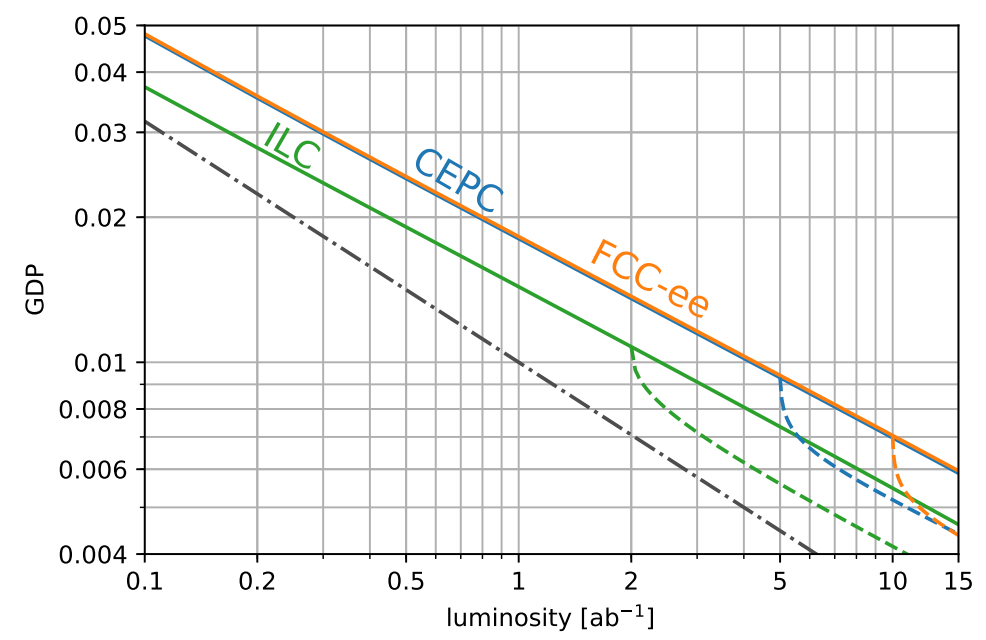

Figure 11. Global GDP strength of the constraints in our eleven-dimensional parameter space as a function the luminosity collected, without beam polarization, at a center-of-mass energy of $240 \mathrm{GeV}$ (for the CEPC and FCC-ee) and at $250 \mathrm{GeV}$, with polarized beams (for the ILC). The dashed lines show the improvements brought by subsequent runs at $350 \mathrm{GeV}$. A pure statistical scaling of constraints, in the absence of systematic uncertainties, would have led to the slope of the dot-dashed line.

with statistics is performed to obtain estimates for other scenarios, assuming no correlation among the measurements carried out with different polarizations. In agreement with the proposal of ref. [58], figure 10 shows that the best overall constraints are obtained with about $70 \%$ of the $2 \mathrm{ab}^{-1} \mathrm{ILC}$ luminosity at $250 \mathrm{GeV}$ spent with $P\left(e^{-}, e^{+}\right)=(-0.8,+0.3)$ beam polarization and $30 \%$ with $P\left(e^{-}, e^{+}\right)=(+0.8,-0.3)$. The $(-0.8,+0.3)$ polarization enhances the cross section, while the $(+0.8,-0.3)$ one helps resolving degeneracies. In terms of GDP, this optimal repartition of luminosity provides results that are $14 \%$ better than a full run with $P\left(e^{-}, e^{+}\right)=(-0.8,+0.3)$ beam polarization. For comparison, we also show the reach of a $240 \mathrm{GeV}$ CEPC run with $5 \mathrm{ab}^{-1}$ of integrated luminosity and unpolarized beams. The higher luminosity is able compensate for the lack of polarization and comparable overall results are obtained. This is further quantified by figure 11 which displays the GDP of our eleven-parameter fit as a function of luminosity collected at $250 \mathrm{GeV}$ with polarized beams and at $240 \mathrm{GeV}$ with unpolarized ones. It is notably seen that only about $1.5 \mathrm{ab}^{-1}$ of additional luminosity are required without polarized beams to match the overall performance obtained with $2 \mathrm{ab}^{-1}$ of polarized beams. With $5 \mathrm{ab}^{-1}$ and $10 \mathrm{ab}^{-1}$ collected at $240 \mathrm{GeV}$, the CEPC and FCC-ee reach GDPs respectively $14 \%$ and $34 \%$ smaller than that of the full ILC run $\left(2 \mathrm{ab}^{-1}\right)$ at $250 \mathrm{GeV}$.

Impact of systematic uncertainties in diboson production. Another important issue concerns the impact of systematic uncertainties on the constraints deriving from $e^{+} e^{-} \rightarrow W W$ measurements. As discussed earlier, they have not yet been determined by dedicated experimental studies except for the $500 \mathrm{GeV}$ ILC run. The top panel of 

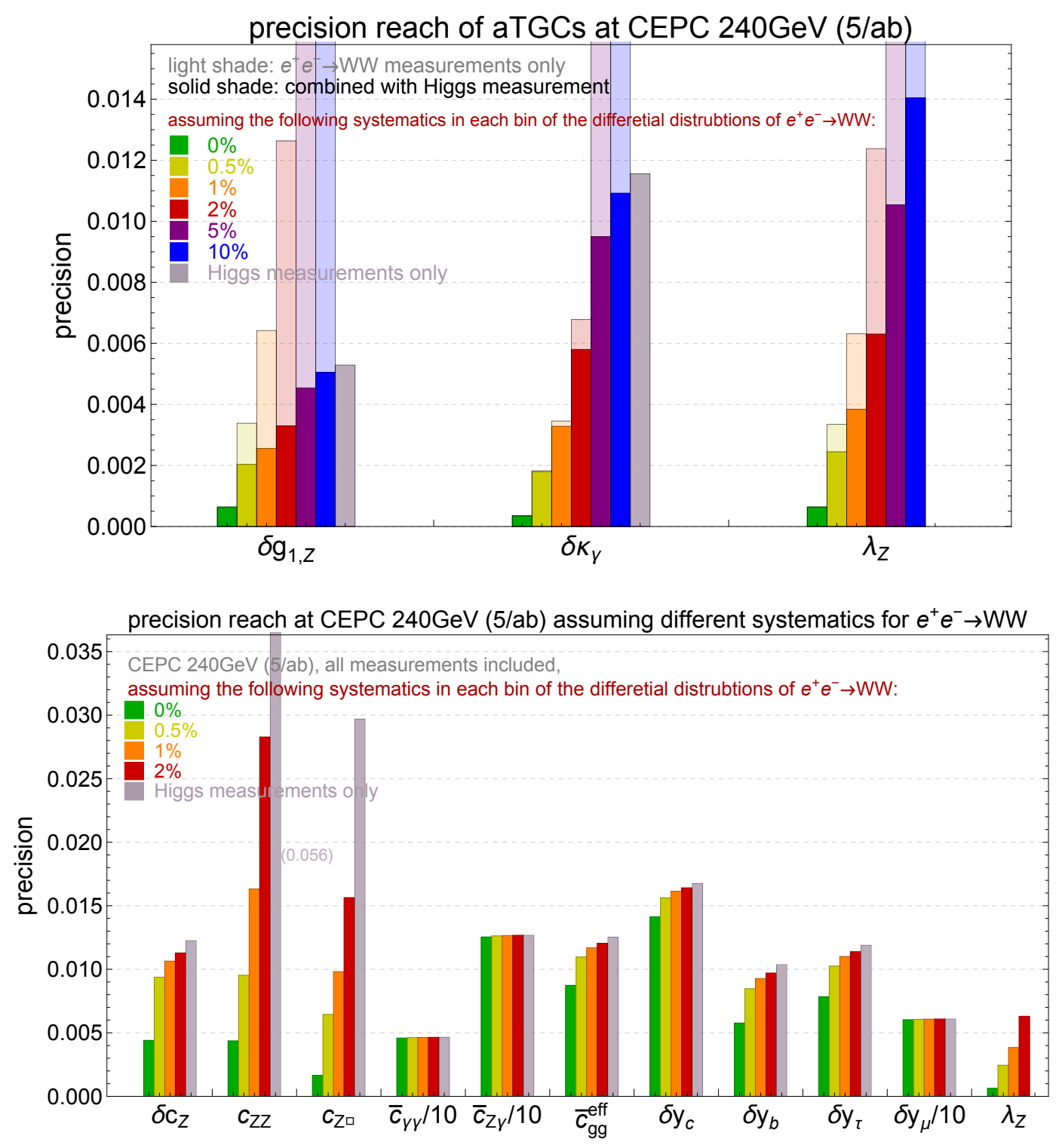

Figure 12. Top: one-sigma constraints on aTGCs parameters for different assumptions about the systematic uncertainties affecting the $e^{+} e^{-} \rightarrow W W$ measurements at the CEPC. Each of the five angular distributions is divided into 20 bins (or 10 bins for the angles characterizing $W$ decays in indistinguishable quark-antiquark pairs). We assume a fixed relative uncertainty each bin, and no correlation among them. A benchmark value of $1 \%$ is used elsewhere in this paper, for CEPC and FCC-ee measurements. Bottom: one-sigma reach of the $240 \mathrm{GeV}$ CEPC run for different systematic uncertainties in the differential measurements of diboson production. 
figure 12 focuses on the aTGC parameters $\delta g_{1, Z}, \delta \kappa_{\gamma}$, and $\lambda_{Z}$. Systematic uncertainties ranging between 0 and $10 \%$ are assumed in each bin of the $e^{+} e^{-} \rightarrow W W \rightarrow 4 f$ angular distributions. The constraints derived from diboson production only are shown in lighter shades. Darker shades show their combination with Higgs measurements, which alone give the gray limits (leaving $\lambda_{Z}$ unconstrained). It is noted that the Higgs measurement constraints on these TGC parameters are improved as soon as uncertainties fall below $10 \%$. On the other hand, Higgs measurements still bring improvements to aTGCs determination when systematic uncertainties fall to $0.5 \%$. The improvement on the whole fit brought by the combination of Higgs and $e^{+} e^{-} \rightarrow W W \rightarrow 4 f$ measurements of varying systematic uncertainties is displayed in figure 12. As shown in eq. (A.6), two of the TGC parameters, $\delta g_{1, Z}$, and $\delta \kappa_{\gamma}$, are related to $c_{Z Z}, c_{Z \square}, c_{\gamma \gamma}, c_{Z \gamma}$ in Higgs measurements. The constraints on the $c_{Z Z}, c_{Z \square}$, and $\lambda_{Z}$ effective-field-theory parameters are benefiting the most from a reduction of systematic uncertainties in diboson production measurements. Improvements are limited for $c_{\gamma \gamma}$ and $c_{Z \gamma}$ which are already well constrained by the measurements of the $h \rightarrow \gamma \gamma$ and $h \rightarrow Z \gamma$ decays.

As our conservative estimate of systematic uncertainties often render the $e^{+} e^{-} \rightarrow W W$ measurements systematics dominated, we show for comparison the results that would have been obtained with perfect TGC constraints with red stars on figure 7 . The figure 13 in appendix $\mathrm{C}$ also contains more detailed fit results under this assumption.

Comparison with previous global analyses. Comparing our results with the ones in refs. [32, 35], a few important differences should be noted. Different assumptions have been made for the run plans of some colliders. In particular, we adopted the most recent ILC run scenario for ILC described in refs. [57, 58], while ref. [32] assumed only $250 \mathrm{fb}^{-1}$ would be collected at $250 \mathrm{GeV}$. Contrarily to refs. [32, 35], we also lift the flavor-universality assumption on Yukawa modifications. Information about the $e^{+} e^{-} \rightarrow h Z$ angular distributions were not included in these existing global analyses. Our respective treatments for the TGC measurements, for which no dedicated experimental study has been carried out, is also different. Reference [35] also addressed the potential of CLIC in probing the Higgsstrahlung process and measuring TGCs at 1.4 and $3 \mathrm{TeV}$. Their estimations for the measurements of $\sigma(h Z) \times \mathrm{BR}(h \rightarrow b \bar{b})$ at 1.4 and $3 \mathrm{TeV}$ are adopted in our study.

\section{Conclusions}

Future lepton colliders running at center-of-mass energies of around $240 \mathrm{GeV}$ and above are ideal to narrow down the Higgs boson properties, examine the fine details of the electroweak symmetry breaking mechanism and indirectly reveal new physics. Applicable in a low-energy limit, the standard-model effective field theory provides a consistent modelindependent framework to parametrize systematically the theory space in direct vicinity of the standard model. We performed, in this paper, a global effective-field-theory analysis of measurements planned at the CEPC, ILC, FCC-ee and CLIC. A basis-independent metric, GDP ratios, was introduced to assess the global strengthening of constraints obtained in different scenarios. We stressed that a consistent effective-field-theory treatment should be 
global and that the combination of various measurements is crucial to constrain effectively all directions of its multidimensional parameter space. We considered the $e^{+} e^{-} \rightarrow h Z$ rates in its various channels as well as angular distributions, the measurement of Higgs production through weak-boson fusion, and that of weak-boson pair production sensitive to anomalous triple gauge couplings which are related to Higgs interactions. Under assumptions discussed in detail, a twelve-dimensional parameter space describes effectivefield-theory contributions to the above observables. We demonstrated that measurements carried out at different center-of-mass energies, or with different beam polarizations, are very effective in resolving approximate degeneracies among effective-field-theory parameters. While circular colliders could collect more luminosity, their linear analogues can reach higher center-of-mass energies and implement longitudinal beam polarizations. High luminosities collected at hadron collider where production rates are often much larger also help constraining rare but clean processes. In that matter, future circular lepton colliders are likely to give way to the next generation of proton colliders reaching $100 \mathrm{TeV}$ center-of-mass energies (the SppC after CEPC [1] and FCC-hh after FCC-ee [56]).

Several improvements to the present analysis are possible. Considering a more complete set of differential observables could obviously strengthen slightly the overall constraints. Quantities that suffer from reduced statistical or systematical uncertainties could also be studied. An important issue which remains to be examined in details concerns the loop-level handles available to constrain the top Yukawa operator below the $t \bar{t} h$ threshold, and around Higgsstrahlung cross section peak in particular. What impact our limited knowledge of electroweak precision observables would have in a global analysis is also a question that should be addressed. Runs at the $Z$ pole or $W W$ threshold may indeed be required to take full advantage of the higher-energy ones. Finally, experimental studies of the weak-boson pair production would be highly valuable to make realistic estimates of the physics potential of future lepton colliders in probing electroweak symmetry breaking.

\section{Acknowledgments}

We thank Adam Falkowski, Zhen Liu, Marc Montull, Michael Peskin, Manqi Ruan, Wei $\mathrm{Su}$, Lian-Tao Wang and Xiangpeng Wang for useful discussions, and James Brau, John Ellis, Jenny List, Zhen Liu, Philipp Roloff, Veronica Sanz and Tevong You for valuable comments on the manuscript. We thank Jürgen Reuter and Jenny List for pointing out the importance of the ILC runs at higher energy, Michelangelo Mangano for suggestions on the normalization of the plots, and Thibaud Vantalon for providing us with quadratic EFT dependences. CG is supported by the European Commission through the Marie Curie Career Integration Grant 631962 and by the Helmholtz Association through the recruitment initiative. JG and KW are supported by an International Postdoctoral Exchange Fellowship Program between the Office of the National Administrative Committee of Postdoctoral Researchers of China (ONACPR) and DESY. We also thank the Collaborative Research Center SFB676 of the Deutsche Forschungsgemeinschaft (DFG), Particles, Strings and the Early Universe, for support. 


\section{A Effective-field-theory parameter definitions}

We define here our effective-field-theory parameters which are closely related to that of the Higgs basis [39]. As explained in section 2, our framework is based on that of refs. [16, $23]$ where electroweak precision observables are assumed to be standard-model like, and where fermion dipole as well as CP-odd operators are discarded. The assumption of flavor universality for Yukawa operators is however relaxed and we include possible modifications of that of the top, charm, bottom, tau, and muon. The expression of our twelve effectivefield-theory parameters in the SILH' basis of dimension-six operators is provided at the end of this section.

The relevant terms in the potential are

$$
\mathcal{L} \supset \mathcal{L}_{h V V}+\mathcal{L}_{h f f}+\mathcal{L}_{\text {tgc }},
$$

where the coupling of Higgs boson to a pair of SM gauge bosons are given by

$$
\begin{aligned}
\mathcal{L}_{h V V}=\frac{h}{v} & {\left[\left(1+\delta c_{W}\right) \frac{g^{2} v^{2}}{2} W_{\mu}^{+} W_{\mu}^{-}+\left(1+\delta c_{Z}\right) \frac{\left(g^{2}+g^{\prime 2}\right) v^{2}}{4} Z_{\mu} Z_{\mu}\right.} \\
& +c_{W W} \frac{g^{2}}{2} W_{\mu \nu}^{+} W_{\mu \nu}^{-}+c_{W \square} g^{2}\left(W_{\mu}^{-} \partial_{\nu} W_{\mu \nu}^{+}+\text {h.c. }\right) \\
& +c_{g g} \frac{g_{s}^{2}}{4} G_{\mu \nu}^{a} G_{\mu \nu}^{a}+c_{\gamma \gamma} \frac{e^{2}}{4} A_{\mu \nu} A_{\mu \nu}+c_{Z \gamma} \frac{e \sqrt{g^{2}+g^{\prime 2}}}{2} Z_{\mu \nu} A_{\mu \nu} \\
& \left.+c_{Z Z} \frac{g^{2}+g^{\prime 2}}{4} Z_{\mu \nu} Z_{\mu \nu}+c_{Z \square} g^{2} Z_{\mu} \partial_{\nu} Z_{\mu \nu}+c_{\gamma \square g g^{\prime}} Z_{\mu} \partial_{\nu} A_{\mu \nu}\right] .
\end{aligned}
$$

Not all the couplings in eq. (A.2) are independent. In particular, imposing gauge invariance, we rewrite the following couplings as

$$
\begin{aligned}
\delta c_{W} & =\delta c_{Z}+4 \delta m, \\
c_{W W} & =c_{Z Z}+2 s_{\theta_{W}}^{2} c_{Z \gamma}+s_{\theta_{W}}^{4} c_{\gamma \gamma}, \\
c_{W \square} & =\frac{1}{g^{2}-g^{\prime 2}}\left[g^{2} c_{Z \square}+g^{\prime 2} c_{Z Z}-e^{2} s_{\theta_{W}}^{2} c_{\gamma \gamma}-\left(g^{2}-g^{\prime 2}\right) s_{\theta_{W}}^{2} c_{Z \gamma}\right], \\
c_{\gamma \square} & =\frac{1}{g^{2}-g^{\prime 2}}\left[2 g^{2} c_{Z \square}+\left(g^{2}+g^{\prime 2}\right) c_{Z Z}-e^{2} c_{\gamma \gamma}-\left(g^{2}-g^{\prime 2}\right) c_{Z \gamma}\right],
\end{aligned}
$$

where $\delta m$ parameterizes custodial symmetry breaking effects which are set to zero in our framework. While the modifications of Yukawa couplings are, in general, $3 \times 3$ complex matrices in generation space, we only consider the diagonal elements relevant for the measurements considered, which are

$$
\mathcal{L}_{h f f}=-\frac{h}{v} \sum_{f=t, c, b, \tau, \mu} m_{f}\left(1+\delta y_{f}\right) \bar{f}_{R} f_{L}+\text { h.c. }
$$


Finally, the triple gauge couplings are given by

$$
\begin{aligned}
\mathcal{L}_{\mathrm{tgc}}= & i g s_{\theta_{W}} A^{\mu}\left(W^{-\nu} W_{\mu \nu}^{+}-W^{+\nu} W_{\mu \nu}^{-}\right) \\
& +i g\left(1+\delta g_{1}^{Z}\right) c_{\theta_{W}} Z^{\mu}\left(W^{-\nu} W_{\mu \nu}^{+}-W^{+\nu} W_{\mu \nu}^{-}\right) \\
& +i g\left[\left(1+\delta \kappa_{Z}\right) c_{\theta_{W}} Z^{\mu \nu}+\left(1+\delta \kappa_{\gamma}\right) s_{\theta_{W}} A^{\mu \nu}\right] W_{\mu}^{-} W_{\nu}^{+} \\
& +\frac{i g}{m_{W}^{2}}\left(\lambda_{Z} c_{\theta_{W}} Z^{\mu \nu}+\lambda_{\gamma} s_{\theta_{W}} A^{\mu \nu}\right) W_{v}^{-\rho} W_{\rho \mu}^{+},
\end{aligned}
$$

where $V_{\mu \nu} \equiv \partial_{\mu} V_{\nu}-\partial_{\nu} V_{\mu}$ for $V=W^{ \pm}, Z, A$. Imposing gauge invariance, one obtains $\delta \kappa_{Z}=\delta g_{1, Z}-t_{\theta_{W}}^{2} \delta \kappa_{\gamma}$ and $\lambda_{Z}=\lambda_{\gamma}$, and the contribution from NP can be parameterized by 3 aTGCs, $\delta g_{1, Z}, \delta \kappa_{\gamma}$ and $\lambda_{Z} \cdot \delta g_{1, Z}$ and $\delta \kappa_{\gamma}$ are related to the Higgs observables and can be expressed as

$$
\begin{aligned}
\delta g_{1, Z} & =\frac{1}{2\left(g^{2}-g^{\prime 2}\right)}\left[-g^{2}\left(g^{2}+g^{\prime 2}\right) c_{Z \square}-g^{\prime 2}\left(g^{2}+g^{\prime 2}\right) c_{Z Z}+e^{2} g^{\prime 2} c_{\gamma \gamma}+g^{\prime 2}\left(g^{2}-g^{\prime 2}\right) c_{Z \gamma}\right] \\
\delta \kappa_{\gamma} & =-\frac{g^{2}}{2}\left(c_{\gamma \gamma} \frac{e^{2}}{g^{2}+g^{\prime 2}}+c_{Z \gamma} \frac{g^{2}-g^{\prime 2}}{g^{2}+g^{\prime 2}}-c_{Z Z}\right) .
\end{aligned}
$$

To summarize, under the assumptions we make, the contribution from dimension-six operators to the potential in eq. (A.1) can be parameterized by the following non-redundant set of 12 parameters:

$$
\delta c_{Z}, \quad c_{Z Z}, \quad c_{Z \square}, \quad c_{\gamma \gamma}, \quad c_{Z \gamma}, \quad c_{g g}, \quad \delta y_{t}, \quad \delta y_{c}, \quad \delta y_{b}, \quad \delta y_{\tau}, \quad \delta y_{\mu}, \quad \lambda_{Z} .
$$

It is straightforward to translate results obtained in the Higgs basis to other bases of dimension-six operators. While all non-redundant basis are equivalent, we found the one listed in table 1 particularly convenient under our assumption that the to $Z$-pole and $W$-mass measurements are perfectly standard-model like. In this basis, the 12 parameters of eq. (A.7) are replaced by the following ones,

$$
\begin{aligned}
\mathcal{L}_{\mathrm{D} 6}= & \frac{c_{H}}{v^{2}} \mathcal{O}_{H}+\frac{\kappa_{W W}}{m_{W}^{2}} \mathcal{O}_{W W}+\frac{\kappa_{B B}}{m_{W}^{2}} \mathcal{O}_{B B}+\frac{\kappa_{H W}}{m_{W}^{2}} \mathcal{O}_{H W}+\frac{\kappa_{H B}}{m_{W}^{2}} \mathcal{O}_{H B} \\
& +\frac{\kappa_{G G}}{m_{W}^{2}} \mathcal{O}_{G G}+\frac{\kappa_{3 W}}{m_{W}^{2}} \mathcal{O}_{3 W}+\sum_{f=t, c, b, \tau, \mu} \frac{c_{y_{f}}}{v^{2}} \mathcal{O}_{y_{f}},
\end{aligned}
$$

where the normalization of the parameters are also defined. To go from the SILH' basis [13, 14] to the one in table 1 , one simply trades $\mathcal{O}_{W}, \mathcal{O}_{B} \rightarrow \mathcal{O}_{W W}, \mathcal{O}_{W B}$ using

$$
\begin{aligned}
\mathcal{O}_{B} & =\mathcal{O}_{H B}+\frac{1}{4} \mathcal{O}_{B B}+\frac{1}{4} \mathcal{O}_{W B}, \\
\mathcal{O}_{W} & =\mathcal{O}_{H W}+\frac{1}{4} \mathcal{O}_{W W}+\frac{1}{4} \mathcal{O}_{W B},
\end{aligned}
$$

where $\mathcal{O}_{W B}$ is directly related to the $Z$-pole measurements ( $S$-parameter) and is thus eliminated. The basis in table 1 is also used in ref. [17] with a different notation. In particular, the $\mathcal{O}_{H W}$ and $\mathcal{O}_{H B}$ in table 1 are denoted as $\mathcal{O}_{W}$ and $\mathcal{O}_{B}$ in those references, which are different from the $\mathcal{O}_{W}$ and $\mathcal{O}_{B}$ in the SILH convention. 


\begin{tabular}{|l|l|}
\hline $\mathcal{O}_{H}=\frac{1}{2}\left(\partial_{\mu}\left|H^{2}\right|\right)^{2}$ & $\mathcal{O}_{G G}=g_{s}^{2}|H|^{2} G_{\mu \nu}^{A} G^{A, \mu \nu}$ \\
$\mathcal{O}_{W W}=g^{2}|H|^{2} W_{\mu \nu}^{a} W^{a, \mu \nu}$ & $\mathcal{O}_{y_{u}}=y_{u}|H|^{2} \bar{Q}_{L} \tilde{H} u_{R}$ \\
$\mathcal{O}_{B B}=g^{\prime 2}|H|^{2} B_{\mu \nu} B^{\mu \nu}$ & $\mathcal{O}_{y_{d}}=y_{d}|H|^{2} \bar{Q}_{L} H d_{R}$ \\
$\mathcal{O}_{H W}=i g\left(D^{\mu} H\right)^{\dagger} \sigma^{a}\left(D^{\nu} H\right) W_{\mu \nu}^{a}$ & $\mathcal{O}_{y_{e}}=y_{e}|H|^{2} \bar{L}_{L} H e_{R}$ \\
$\mathcal{O}_{H B}=i g^{\prime}\left(D^{\mu} H\right)^{\dagger}\left(D^{\nu} H\right) B_{\mu \nu}$ & $\mathcal{O}_{3 W}=\frac{1}{3 !} g \epsilon_{a b c} W_{\mu}^{a \nu} W_{\nu \rho}^{b} W^{c \rho \mu}$ \\
\hline
\end{tabular}

Table 1. A complete set of CP-even dimension-six operators that contribute to the Higgs and TGC measurements, assuming there is no correction to the $Z$-pole and $W$ mass measurements and no dipole interaction. We only consider the flavor-conserving component of $\mathcal{O}_{y_{u}}, \mathcal{O}_{y_{d}}$ and $\mathcal{O}_{y_{e}}$ contributing to the top, charm, bottom, tau, and muon Yukawa couplings.

The aTGCs in this basis are given by

$$
\begin{aligned}
\delta g_{1, Z} & =-\frac{\kappa_{H W}}{c_{\theta_{W}}^{2}}, \\
\delta \kappa_{\gamma} & =-\kappa_{H W}-\kappa_{H B}, \\
\lambda_{Z} & =-\kappa_{3 W}
\end{aligned}
$$

which are obtained from the general results in ref. [21]. Finally, the expression of our effective-field-theory parameters in terms of the operators in table 1 are:

$$
\begin{aligned}
\delta c_{Z} & =-\frac{1}{2} c_{H}, \\
c_{Z Z} & =\frac{4}{g^{2}+g^{\prime 2}}\left(-\kappa_{H W}-t_{\theta_{W}}^{2} \kappa_{H B}+4 c_{\theta_{W}}^{2} \kappa_{W W}+4 t_{\theta_{W}}^{2} s_{\theta_{W}}^{2} \kappa_{B B}\right), \\
c_{Z \square} & =\frac{2}{g^{2}}\left(\kappa_{H W}+t_{\theta_{W}}^{2} \kappa_{H B}\right), \\
c_{\gamma \gamma} & =\frac{16}{g^{2}}\left(\kappa_{W W}+\kappa_{B B}\right), \\
c_{Z \gamma} & =\frac{2}{g^{2}}\left(\kappa_{H B}-\kappa_{H W}+8 c_{\theta_{W}}^{2} \kappa_{W W}-8 s_{\theta_{W}}^{2} \kappa_{B B}\right), \\
c_{g g} & =\frac{16}{g^{2}} \kappa_{G G}, \\
\delta y_{f} & =-\frac{1}{2} c_{H}-c_{y_{f}} .
\end{aligned}
$$

It should be noted that eq. (A.11) is only valid under the assumptions made in this paper. More general basis translations from the Higss basis to the SILH' basis (and others) are provided in ref. [39].

\section{B Measurement inputs}

We provide here additional details about the input measurements used in our study, including the Higgs production rates $\left(e^{+} e^{-} \rightarrow h Z\right.$ and $\left.e^{+} e^{-} \rightarrow \nu \bar{\nu} h\right)$, the angular asymmetries 
in $e^{+} e^{-} \rightarrow h Z$ and TGC measurements from $e^{+} e^{-} \rightarrow W W$. The estimated one-sigma precisions of the Higgs rate measurements are respectively displayed in table 2 for the CEPC and FCC-ee, in table 3 for ILC and, in table 4 for CLIC. When provided, the are respectively extracted from ref. [86] for the CEPC (which updates the preCDR [1]), ref. [2] for the FCC-ee, ref. [58] for the ILC and ref. [60] for CLIC. For CLIC, we also include the estimations for $\sigma(h Z) \times \mathrm{BR}(h \rightarrow b \bar{b})$ at 1.4 and $3 \mathrm{TeV}$ from ref. [35]. While these measurements suffer from smaller cross sections, they nevertheless significantly improve the constraints on $c_{Z Z}$ and $c_{Z \square}$ due to the huge sensitivities at high energies. ${ }^{8}$ We also found the $Z Z$ fusion measurements at CLIC (with $\sigma\left(e^{+} e^{-} h\right) \times \mathrm{BR}(h \rightarrow b \bar{b})$ measured to a precision of $1.8 \%$ $(2.3 \%)$ at $1.4 \mathrm{TeV}(3 \mathrm{TeV})[60])$ to have a negligible impact in our analysis. ${ }^{9}$ The numbers highlighted in green are obtained by scaling with luminosity when dedicated estimates are not available. For the ILC, the estimations of signal strengths are summarized in ref. [58] (table 13) but only for benchmark run scenarios with smaller luminosities. These are scaled up to the current run plan. For the $350 \mathrm{GeV}$ run of CEPC and FCC-ee, relative precision are rescaled from the $350 \mathrm{GeV}$ ILC ones. ${ }^{10}$ The precision of $\sigma(h Z) \times \mathrm{BR}(h \rightarrow Z \gamma)$ is not provided for the FCC-ee and ILC. We thus scale it from the CEPC estimation. While a statistical precision of $2.2 \%$ is reported in ref. [2] for the $\sigma(\nu \bar{\nu} h) \times \mathrm{BR}(h \rightarrow b \bar{b})$ measurement at FCC-ee $240 \mathrm{GeV}$, it is not clear what assumptions on the $e^{+} e^{-} \rightarrow h Z, Z \rightarrow \nu \bar{\nu}$ process are made in obtaining this estimation. Therefore, we scale it with luminosity from the CEPC one. The difference between unpolarized and polarized cross sections are taken into account in these rescalings. Given the moderate statistics in most of the relevant channels, it is reasonable to assume their precision is statistics limited. Nevertheless, it is important for these estimations to be updated by experimental groups in the future.

The constraints from angular observables in $e^{+} e^{-} \rightarrow h Z$ are obtained with the method described in section 3.1, making use of the channels $e^{+} e^{-} \rightarrow h Z, Z \rightarrow \ell^{+} \ell^{-}, h \rightarrow b \bar{b}, c \bar{c}$, $g g$. They are included for all the $e^{+} e^{-}$colliders at all energies except for the $1.4 \mathrm{TeV}$ and $3 \mathrm{TeV}$ runs of CLIC.

The constraints on aTGCs derived from the $e^{+} e^{-} \rightarrow W W$ measurements are obtained using the method described in section 3.4, for the CEPC and FCC-ee. In particular, 1\% systematic uncertainties are assumed in each bin with the differential distribution of each measured angle divided in 20 bins (10 bins if the angle is folded). The results, including the correlation matrices, are shown in table 5 and table 6 , which are fed into the global fit. For ILC, the constraints are shown in table 7, taken from ref. [68], which assumes $500 \mathrm{fb}^{-1}$ data at $500 \mathrm{GeV}$ and four $P\left(e^{-}, e^{+}\right)=( \pm 0.8, \pm 0.3)$ beam polarization configurations. For CLIC, we simply use the ILC results.

While the measurement inputs of LHC and LEP measurements are too lengthy to be reported in this paper, here we simply list the results from the global fits in terms of one sigma constraints and the correlation matrix, which can be used to reconstruct the chi-

\footnotetext{
${ }^{8}$ We thank Tevong You for pointing this out.

${ }^{9}$ It is nevertheless possible to further optimize the precision reach of the cross section measurements of $Z Z$ fusion using judicious kinematic cuts, as pointed out in ref. [87]. For simplicity, we do not perform such optimizations in our study.

${ }^{10} \mathrm{~A}$ statistical precision of $0.6 \%$ is reported in ref. [2] for the $\sigma(\nu \bar{\nu} h) \times \mathrm{BR}(h \rightarrow b \bar{b})$ measurement at FCC-ee $350 \mathrm{GeV}$, which is in good agreement with our estimation from scaling $(0.71 \%)$.
} 


\begin{tabular}{|c|cc|cc|cc|cc|}
\hline & \multicolumn{5}{|c|}{ CEPC } & \multicolumn{4}{c|}{ FCC-ee } \\
\hline & {$\left[240 \mathrm{GeV}, 5 \mathrm{ab}^{-1}\right]$} & {$\left[350 \mathrm{GeV}, 200 \mathrm{fb}^{-1}\right]$} & {$\left[240 \mathrm{GeV}, 10 \mathrm{ab}^{-1}\right]$} & {$\left[350 \mathrm{GeV}, 2.6 \mathrm{ab}^{-1}\right]$} \\
\hline production & $Z h$ & $\nu \bar{\nu} h$ & $Z h$ & $\nu \bar{\nu} h$ & $Z h$ & $\nu \bar{\nu} h$ & $Z h$ & $\nu \bar{\nu} h$ \\
\hline$\sigma$ & $0.50 \%$ & - & $2.4 \%$ & - & $0.40 \%$ & - & $0.67 \%$ & - \\
\hline & \multicolumn{3}{|c|}{$\sigma \times \mathrm{BR}$} \\
\hline$h \rightarrow b \bar{b}$ & $0.21 \%{ }^{\star}$ & $0.39 \%$ & $2.0 \%$ & $2.6 \%$ & $0.20 \%$ & $0.28 \% \diamond$ & $0.54 \%$ & $0.71 \%$ \\
$h \rightarrow c \bar{c}$ & $2.5 \%$ & - & $15 \%$ & $26 \%$ & $1.2 \%$ & - & $4.1 \%$ & $7.1 \%$ \\
$h \rightarrow g g$ & $1.2 \%$ & - & $11 \%$ & $17 \%$ & $1.4 \%$ & - & $3.1 \%$ & $4.7 \%$ \\
$h \rightarrow \tau \tau$ & $1.0 \%$ & - & $5.3 \%$ & $37 \%$ & $0.7 \%$ & - & $1.5 \%$ & $10 \%$ \\
$h \rightarrow W W^{*}$ & $1.0 \%$ & - & $10 \%$ & $9.8 \%$ & $0.9 \%$ & - & $2.8 \%$ & $2.7 \%$ \\
$h \rightarrow Z Z^{*}$ & $4.3 \%$ & - & $33 \%$ & $33 \%$ & $3.1 \%$ & - & $9.2 \%$ & $9.3 \%$ \\
$h \rightarrow \gamma \gamma$ & $9.0 \%$ & - & $51 \%$ & $77 \%$ & $3.0 \%$ & - & $14 \%$ & $21 \%$ \\
$h \rightarrow \mu \mu$ & $12 \%$ & - & $115 \%$ & $275 \%$ & $13 \%$ & - & $32 \%$ & $76 \%$ \\
$h \rightarrow Z \gamma$ & $25 \%$ & - & $144 \%$ & - & $18 \%$ & - & $40 \%$ & - \\
\hline
\end{tabular}

Table 2. The estimated precision of CEPC and FCC-ee Higgs measurements. We gather the available estimations from refs. [1, 2, 86], while the missing ones (highlighted in green) are obtained from scaling with luminosity. See appendix B for more details. For $\sigma\left(e^{+} e^{-} \rightarrow \nu \bar{\nu} h\right)$, the precisions marked with a diamond $\diamond$ are normalized to the cross section of the inclusive channel which includes both the $W W$ fusion and $e^{+} e^{-} \rightarrow h Z, Z \rightarrow \nu \bar{\nu}$, while the unmarked precisions are normalized to the $W W$ fusion process only. For the CEPC, the precision of the $\sigma(h Z) \times \mathrm{BR}(h \rightarrow b \bar{b})$ measurement (marked by a star ${ }^{\star}$ ) reduces to $0.24 \%$ if one excludes the contribution from $e^{+} e^{-} \rightarrow h Z, Z \rightarrow$ $\nu \bar{\nu}, h \rightarrow b \bar{b}$ to avoid double counting with $e^{+} e^{-} \rightarrow \nu \bar{\nu} h, h \rightarrow b \bar{b}$. The corresponding information is not available for the FCC-ee.

\begin{tabular}{|c|c|c|c|c|c|c|c|c|c|c|c|}
\hline \multicolumn{12}{|c|}{ ILC } \\
\hline & \multicolumn{2}{|c|}{$\left[250 \mathrm{GeV}, 2 \mathrm{ab}^{-1}\right]$} & \multicolumn{2}{|c|}{$\left[350 \mathrm{GeV}, 200 \mathrm{fb}^{-1}\right]$} & \multicolumn{3}{|c|}{$\left[500 \mathrm{GeV}, 4 \mathrm{ab}^{-1}\right]$} & \multicolumn{2}{|c|}{$\left[1 \mathrm{TeV}, 1 \mathrm{ab}^{-1}\right]$} & \multicolumn{2}{|c|}{$\left[1 \mathrm{TeV}, 2.5 \mathrm{ab}^{-1}\right]$} \\
\hline production & $Z h$ & $\nu \bar{\nu} h$ & $Z h$ & $\nu \bar{\nu} h$ & $Z h$ & $\nu \bar{\nu} h$ & $t \bar{t} h$ & $\nu \bar{\nu} h$ & $t \bar{t} h$ & $\nu \bar{\nu} h$ & $t \bar{t} h$ \\
\hline \multirow[t]{2}{*}{$\sigma$} & $0.71 \%$ & - & $2.1 \%$ & - & $1.1 \%$ & - & - & - & - & - & - \\
\hline & \multicolumn{11}{|c|}{$\sigma \times \mathrm{BR}$} \\
\hline$h \rightarrow b \bar{b}$ & $0.42 \%$ & $3.7 \%$ & $1.7 \%$ & $1.7 \%$ & $0.64 \%$ & $0.25 \%$ & $9.9 \%$ & $0.5 \%$ & $6.0 \%$ & $0.3 \%$ & $3.8 \%$ \\
\hline$h \rightarrow c \bar{c}$ & $2.9 \%$ & - & $13 \%$ & $17 \%$ & $4.6 \%$ & $2.2 \%$ & - & $3.1 \%$ & - & $2.0 \%$ & - \\
\hline$h \rightarrow g g$ & $2.5 \%$ & - & $9.4 \%$ & $11 \%$ & $3.9 \%$ & $1.4 \%$ & - & $2.3 \%$ & - & $1.4 \%$ & - \\
\hline$h \rightarrow \tau \tau$ & $1.1 \%$ & - & $4.5 \%$ & $24 \%$ & $1.9 \%$ & $3.2 \%$ & - & $1.6 \%$ & - & $1.0 \%$ & - \\
\hline$h \rightarrow W W^{*}$ & $2.3 \%$ & - & $8.7 \%$ & $6.4 \%$ & $3.3 \%$ & $0.85 \%$ & - & $3.1 \%$ & - & $2.0 \%$ & - \\
\hline$h \rightarrow Z Z^{*}$ & $6.7 \%$ & - & $28 \%$ & $22 \%$ & $8.8 \%$ & $2.9 \%$ & - & $4.1 \%$ & - & $2.6 \%$ & - \\
\hline$h \rightarrow \gamma \gamma$ & $12 \%$ & - & $44 \%$ & $50 \%$ & $12 \%$ & $6.7 \%$ & - & $8.5 \%$ & - & $5.4 \%$ & - \\
\hline$h \rightarrow \mu \mu$ & $25 \%$ & - & $98 \%$ & $180 \%$ & $31 \%$ & $25 \%$ & - & $31 \%$ & - & $20 \%$ & - \\
\hline$h \rightarrow Z \gamma$ & $34 \%$ & - & $145 \%$ & - & $49 \%$ & - & - & - & - & - & - \\
\hline
\end{tabular}

Table 3. The estimated precision of ILC Higgs measurements. For the $250 \mathrm{GeV}, 350 \mathrm{GeV}$ and $500 \mathrm{GeV}$ runs, all numbers are scaled from ref. [58] (table 13), except for $\sigma(h Z) \times \operatorname{BR}(h \rightarrow Z \gamma)$ which is scaled from the CEPC estimation. A beam polarization of $P\left(e^{-}, e^{+}\right)=(-0.8,+0.3)$ is assumed. The $1 \mathrm{TeV}$ run is only included in figure 17 of appendix $\mathrm{C}$, while the estimations are taken from ref. [59] which assumes a polarization of $P\left(e^{-}, e^{+}\right)=(-0.8,+0.2)$. 


\begin{tabular}{|c|cc|cc|c|}
\hline \multicolumn{5}{|c|}{ CLIC } \\
\hline & {$\left[350 \mathrm{GeV}, 500 \mathrm{fb}^{-1}\right]$} & {$\left[1.4 \mathrm{TeV}, 1.5 \mathrm{ab}^{-1}\right]$} & {$\left[3 \mathrm{TeV}, 2 \mathrm{ab}^{-1}\right]$} \\
\hline production & $Z h$ & $\nu \bar{\nu} h$ & $\nu \bar{\nu} h$ & $t \bar{t} h$ & $\nu \bar{\nu} h$ \\
\hline$\sigma$ & $1.6 \%$ & - & - & - & - \\
\hline & \multicolumn{5}{|c|}{$\sigma \times \mathrm{BR}$} \\
\hline$h \rightarrow b \bar{b}$ & $0.84 \%$ & $1.9 \%$ & $0.4 \%$ & $8.4 \%$ & $0.3 \%$ \\
$h \rightarrow c \bar{c}$ & $10.3 \%$ & $14.3 \%$ & $6.1 \%$ & - & $6.9 \%$ \\
$h \rightarrow g g$ & $4.5 \%$ & $5.7 \%$ & $5.0 \%$ & - & $4.3 \%$ \\
$h \rightarrow \tau \tau$ & $6.2 \%$ & - & $4.2 \%$ & - & $4.4 \%$ \\
$h \rightarrow W W^{*}$ & $5.1 \%$ & - & $1.0 \%$ & - & $0.7 \%$ \\
$h \rightarrow Z Z^{*}$ & - & - & $5.6 \%$ & - & $3.9 \%$ \\
$h \rightarrow \gamma \gamma$ & - & - & $15 \%$ & - & $10 \%$ \\
$h \rightarrow \mu \mu$ & - & - & $38 \%$ & - & $25 \%$ \\
$h \rightarrow Z \gamma$ & - & - & $42 \%$ & - & $30 \%$ \\
\hline
\end{tabular}

Table 4. The estimated precision of CLIC Higgs measurements taken from ref. [60], which assumes unpolarized beams and considers only statistical uncertainties. In addition, we also include the estimations for $\sigma(h Z) \times \mathrm{BR}(h \rightarrow b \bar{b})$ at high energies in ref. [35], which are $3.3 \%(6.8 \%)$ at $1.4 \mathrm{TeV}$ $(3 \mathrm{TeV})$. We find the inclusion of the $Z Z$ fusion $\left(e^{+} e^{-} \rightarrow e^{+} e^{-} h\right)$ measurements to have little impact in our analysis.

\begin{tabular}{|c|c|ccc|c|ccc|}
\hline \multicolumn{8}{|c|}{ CEPC } \\
\hline \multirow{3}{*}{} & \multicolumn{3}{|c|}{$240 \mathrm{GeV}\left(5 \mathrm{ab}^{-1}\right)$} & \multicolumn{2}{|c|}{$240 \mathrm{GeV}\left(5 \mathrm{ab}^{-1}\right)+350 \mathrm{GeV}\left(200 \mathrm{fb}^{-1}\right)$} \\
\cline { 2 - 9 } & uncertainty & \multicolumn{2}{|c|}{ correlation matrix } & uncertainty & \multicolumn{2}{|c|}{ correlation matrix } \\
\cline { 2 - 9 } & & $\delta g_{1, Z}$ & $\delta \kappa_{\gamma}$ & $\lambda_{Z}$ & & $\delta g_{1, Z}$ & $\delta \kappa_{\gamma}$ & $\lambda_{Z}$ \\
\hline$\delta g_{1, Z}$ & 0.0064 & 1 & 0.068 & -0.93 & 0.0037 & 1 & -0.51 & -0.89 \\
$\delta \kappa_{\gamma}$ & 0.0035 & & 1 & -0.40 & 0.0017 & & 1 & 0.12 \\
$\lambda_{Z}$ & 0.0063 & & & 1 & 0.0030 & & & 1 \\
\hline
\end{tabular}

Table 5. The constraints on aTGCs from the $e^{+} e^{-} \rightarrow W W$ measurement at CEPC using the methods described in section 3.4. Both the results from the $240 \mathrm{GeV}$ run alone and the ones from the combination of the $240 \mathrm{GeV}$ and $350 \mathrm{GeV}$ runs are shown.

\begin{tabular}{|c|c|ccc|c|ccc|}
\hline \multicolumn{7}{|c|}{ FCC-ee } \\
\hline \multirow{3}{*}{} & \multicolumn{3}{|c|}{$240 \mathrm{GeV}\left(10 \mathrm{ab}^{-1}\right)$} & \multicolumn{2}{|c|}{$240 \mathrm{GeV}\left(10 \mathrm{ab}^{-1}\right)+350 \mathrm{GeV}\left(2.6 \mathrm{ab}^{-1}\right)$} \\
\cline { 2 - 9 } & uncertainty & \multicolumn{2}{|c|}{ correlation matrix } & uncertainty & \multicolumn{3}{|c|}{ correlation matrix } \\
\cline { 2 - 9 } & & $\delta g_{1, Z}$ & $\delta \kappa_{\gamma}$ & $\lambda_{Z}$ & & $\delta g_{1, Z}$ & $\delta \kappa_{\gamma}$ & $\lambda_{Z}$ \\
\hline$\delta g_{1, Z}$ & 0.0064 & 1 & 0.066 & -0.93 & 0.0029 & 1 & -0.61 & -0.88 \\
$\delta \kappa_{\gamma}$ & 0.0034 & & 1 & -0.40 & 0.0014 & & 1 & 0.19 \\
$\lambda_{Z}$ & 0.0062 & & & 1 & 0.0022 & & & 1 \\
\hline
\end{tabular}

Table 6. Same as table 5 but for FCC-ee. 


\begin{tabular}{|c|c|ccc|}
\hline \multicolumn{5}{|c|}{ ILC (CLIC) } \\
\hline \multirow{2}{*}{} & uncertainty & \multicolumn{3}{|c|}{ correlation matrix } \\
\cline { 2 - 5 } & & $\delta g_{1, Z}$ & $\delta \kappa_{\gamma}$ & $\lambda_{Z}$ \\
\hline$\delta g_{1, Z}$ & $6.1 \times 10^{-4}$ & 1 & 0.634 & 0.477 \\
$\delta \kappa_{\gamma}$ & $6.4 \times 10^{-4}$ & & 1 & 0.354 \\
$\lambda_{Z}$ & $7.2 \times 10^{-4}$ & & & 1 \\
\hline
\end{tabular}

Table 7. The estimated statistical precision of aTGCs from the $e^{+} e^{-} \rightarrow W W$ measurements at ILC in ref. [68], assuming $500 \mathrm{fb}^{-1}$ of data equally shared between four $P\left(e^{-}, e^{+}\right)=( \pm 0.8, \pm 0.3)$ beam polarization configurations at $500 \mathrm{GeV}$. We use the same results for CLIC. No scaling with statistics or center-of-mass energy is performed, given that systematic uncertainties may become important.

\begin{tabular}{|c|c|c|c|c|c|c|c|c|c|c|c|}
\hline \multicolumn{12}{|c|}{ LHC $8 \mathrm{TeV}$ Higgs + LEP $e^{+} e^{-} \rightarrow W W$} \\
\hline & uncertainty & \multicolumn{10}{|c|}{ correlation matrix } \\
\hline & & $\delta c_{Z}$ & $c_{Z Z}$ & $c_{Z \square}$ & $c_{\gamma \gamma}$ & $c_{Z \gamma}$ & $c_{g g}$ & $\delta y_{u}$ & $\delta y_{d}$ & $\delta y_{e}$ & $\lambda_{Z}$ \\
\hline$\delta c_{Z}$ & 0.17 & 1 & -0.04 & -0.21 & -0.76 & -0.15 & 0.15 & 0.12 & 0.88 & 0.71 & -0.22 \\
\hline$c_{Z Z}$ & 0.42 & & 1 & -0.96 & 0.37 & 0.19 & 0.03 & 0.04 & -0.12 & -0.31 & -0.88 \\
\hline$c_{Z \square}$ & 0.19 & & & 1 & -0.17 & -0.10 & -0.07 & -0.06 & -0.10 & 0.12 & 0.93 \\
\hline$c_{\gamma \gamma}$ & 0.015 & & & & 1 & 0.20 & -0.12 & -0.07 & -0.79 & -0.74 & -0.13 \\
\hline$c_{Z \gamma}$ & 0.098 & & & & & 1 & -0.01 & -0.01 & -0.15 & -0.18 & -0.10 \\
\hline$c_{g g}$ & 0.0027 & & & & & & 1 & -0.87 & 0.26 & 0.17 & -0.07 \\
\hline$\delta y_{u}$ & 0.30 & & & & & & & 1 & 0.13 & 0.11 & -0.06 \\
\hline$\delta y_{d}$ & 0.35 & & & & & & & & 1 & 0.81 & -0.11 \\
\hline$\delta y_{e}$ & 0.20 & & & & & & & & & 1 & 0.09 \\
\hline$\lambda_{Z}$ & 0.073 & & & & & & & & & & 1 \\
\hline
\end{tabular}

Table 8. Current constraints on the Higgs basis parameters from ref. [23], obtained from the LHC $8 \mathrm{TeV}$ Higgs measurements and LEP $e^{+} e^{-} \rightarrow W W$ measurements. Flavor universality is imposed. To transform it into our framework we simply take $\delta y_{u} \rightarrow \delta y_{t}, \delta_{d} \rightarrow \delta y_{b}, \delta y_{e} \rightarrow \delta y_{\tau}$. For consistency we also set the central values to zero.

square. The chi-square can then be combined with the ones of the future $e^{+} e^{-}$colliders to reproduce the results in figure 7 and table 11 . In table 8 , we list the current constraints in ref. [23], obtained from the LHC $8 \mathrm{TeV}$ Higgs measurements and LEP $e^{+} e^{-} \rightarrow W W$ measurements. While ref. [23] explicitly assumes flavor universality for the Yukawa couplings, it is a good approximation to simply assume the constraints given there apply to third-generation couplings. Since we explicitly assume the future results are SM-like, for consistency, we also set the central values of current results to zero when combining them with the future collider results. In table 9 and 10, we list the results for the $14 \mathrm{TeV}$ LHC with $300 \mathrm{fb}^{-1}$ and $3000 \mathrm{fb}^{-1}$ luminosity, derived from projection by the ATLAS collaboration [73] which collected information from various other sources, while the information about the composition of each channel are extracted from refs. [74-78]. While $\delta y_{c}$ is set to zero in obtaining these results (due to the fact that ref. [73] did not provide estimations for the decay $h \rightarrow c \bar{c}$ ), it is not set to zero when the $\chi^{2}$ is combined with the ones from future $e^{+} e^{-}$colliders. However, this has little impact on the results of the combined fits. 


\begin{tabular}{|c|c|c|c|c|c|c|c|c|c|c|c|}
\hline \multicolumn{12}{|c|}{ LHC $14 \mathrm{TeV}$ Higgs measurements $\left(300 \mathrm{fb}^{-1}\right)$} \\
\hline & uncertainty & \multicolumn{10}{|c|}{ correlation matrix } \\
\hline & & $\delta c_{Z}$ & $c_{Z Z}$ & $c_{Z \square}$ & $c_{\gamma \gamma}$ & $c_{Z \gamma}$ & $c_{g g}$ & $\delta y_{t}$ & $\delta y_{b}$ & $\delta y_{\tau}$ & $\delta y_{\mu}$ \\
\hline$\delta c_{Z}$ & 0.116 & 1 & -0.029 & -0.037 & -0.61 & -0.29 & 0.037 & 0.36 & 0.88 & 0.50 & 0.43 \\
\hline$c_{Z Z}$ & 0.960 & & 1 & -0.996 & 0.77 & 0.52 & -0.17 & 0.43 & -0.21 & -0.73 & -0.55 \\
\hline$c_{Z \square}$ & 0.419 & & & 1 & -0.73 & -0.50 & 0.17 & -0.46 & 0.15 & 0.70 & 0.52 \\
\hline$c_{\gamma \gamma}$ & 0.0156 & & & & 1 & 0.57 & -0.18 & 0.13 & -0.69 & -0.85 & -0.68 \\
\hline$c_{Z \gamma}$ & 0.0164 & & & & & 1 & -0.10 & 0.070 & -0.41 & -0.54 & -0.41 \\
\hline$c_{g g}$ & 0.00137 & & & & & & 1 & -0.74 & 0.063 & 0.14 & 0.26 \\
\hline$\delta y_{t}$ & 0.220 & & & & & & & 1 & 0.42 & -0.094 & -0.20 \\
\hline$\delta y_{b}$ & 0.303 & & & & & & & & 1 & 0.61 & 0.47 \\
\hline$\delta y_{\tau}$ & 0.196 & & & & & & & & & 1 & 0.60 \\
\hline$\delta y_{\mu}$ & 0.271 & & & & & & & & & & 1 \\
\hline
\end{tabular}

Table 9. One sigma constraints and the correlation matrix of the Higgs basis parameters from the LHC $14 \mathrm{TeV}$ Higgs measurements with $300 \mathrm{fb}^{-1}$ data, using the ATLAS projection with no theory error [73]. $\delta y_{c}$ to set to zero since ref. [73] did not provide estimations for the decay $h \rightarrow c \bar{c}$.

\begin{tabular}{|c|c|c|c|c|c|c|c|c|c|c|c|}
\hline \multicolumn{12}{|c|}{ LHC $14 \mathrm{TeV}$ Higgs measurements $\left(3000 \mathrm{fb}^{-1}\right)$} \\
\hline & uncertainty & \multicolumn{10}{|c|}{ correlation matrix } \\
\hline & & $\delta c_{Z}$ & $c_{Z Z}$ & $c_{Z \square}$ & $c_{\gamma \gamma}$ & $c_{Z \gamma}$ & $c_{g g}$ & $\delta y_{t}$ & $\delta y_{b}$ & $\delta y_{\tau}$ & $\delta y_{\mu}$ \\
\hline$\delta c_{Z}$ & 0.0500 & 1 & 0.0015 & -0.045 & -0.54 & -0.22 & 0.034 & 0.38 & 0.87 & 0.40 & 0.45 \\
\hline$c_{Z Z}$ & 0.495 & & 1 & -0.998 & 0.81 & 0.46 & -0.19 & 0.55 & -0.22 & -0.62 & -0.68 \\
\hline$c_{Z \square}$ & 0.214 & & & 1 & -0.78 & -0.44 & 0.20 & -0.57 & 0.18 & 0.60 & 0.66 \\
\hline$c_{\gamma \gamma}$ & 0.00738 & & & & 1 & 0.50 & -0.20 & 0.27 & -0.66 & -0.73 & -0.81 \\
\hline$c_{Z \gamma}$ & 0.00935 & & & & & 1 & -0.099 & 0.13 & -0.34 & -0.39 & -0.42 \\
\hline$c_{g g}$ & 0.000462 & & & & & & 1 & -0.65 & 0.086 & 0.13 & 0.26 \\
\hline$\delta y_{t}$ & 0.0856 & & & & & & & 1 & 0.39 & -0.15 & -0.30 \\
\hline$\delta y_{b}$ & 0.125 & & & & & & & & 1 & 0.50 & 0.54 \\
\hline$\delta y_{\tau}$ & 0.114 & & & & & & & & & 1 & 0.61 \\
\hline$\delta y_{\mu}$ & 0.108 & & & & & & & & & & 1 \\
\hline
\end{tabular}

Table 10. Same as table 9 but for $14 \mathrm{TeV} \mathrm{LHC} 3000 \mathrm{fb}^{-1}$. Note that while $\delta y_{c}$ is set to zero in obtaining these results, it is not set to zero when the $\chi^{2}$ is combined with the ones from future $e^{+} e^{-}$colliders. This has little impact on the results of the combined fits.

\section{Additional figures}

Here we provide additional results of the global fits. In our study, conservative estimates have been made for the measurements of the diboson process $\left(e^{+} e^{-} \rightarrow W W\right)$ which often end up being systematics dominated. To give a sense of the impact of these systematic uncertainties we show, in figure 13, global fit results in which aTGCs are assumed to be perfectly constrained.

Figure 14 reproduces the results in figure 7 in the basis defined by eq. (A.8) and table 1. The analogues to the figures presented in the main text for the CEPC, figure 8-12, are given 


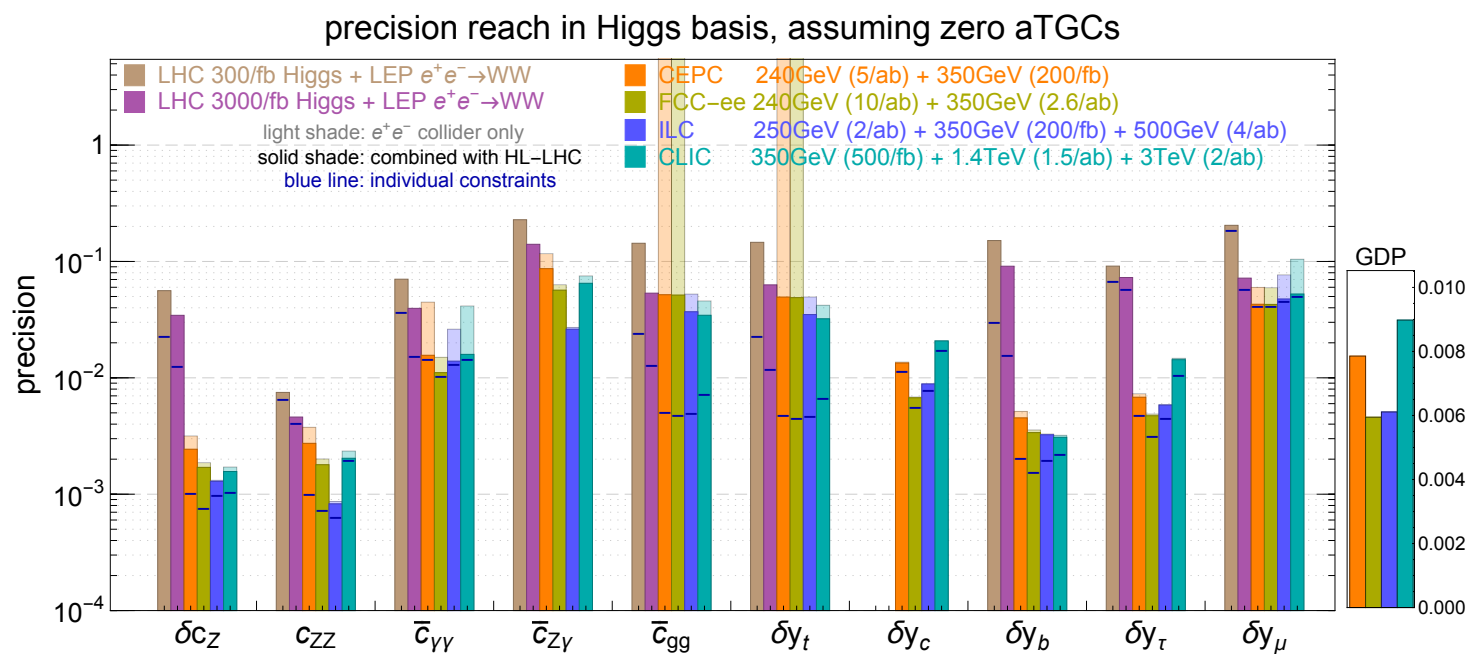

Figure 13. Same as figure 7 but assuming vanishing aTGCs. Imposing $\delta g_{1, Z}=\delta \kappa_{\gamma}=\lambda_{Z}=0$, both $\lambda_{Z}$ and $c_{Z \square}$ are eliminated, while the relation $e^{2} c_{\gamma \gamma}+\left(g^{2}-g^{\prime 2}\right) c_{Z \gamma}-\left(g^{2}+g^{\prime 2}\right) c_{Z Z}=0$ is imposed among $c_{Z Z}, c_{\gamma \gamma}$ and $c_{Z \gamma}$. Note that the individual constraints are basis dependent. We use the above relation to eliminate $c_{Z \gamma}$, hence its individual constraints are not shown.

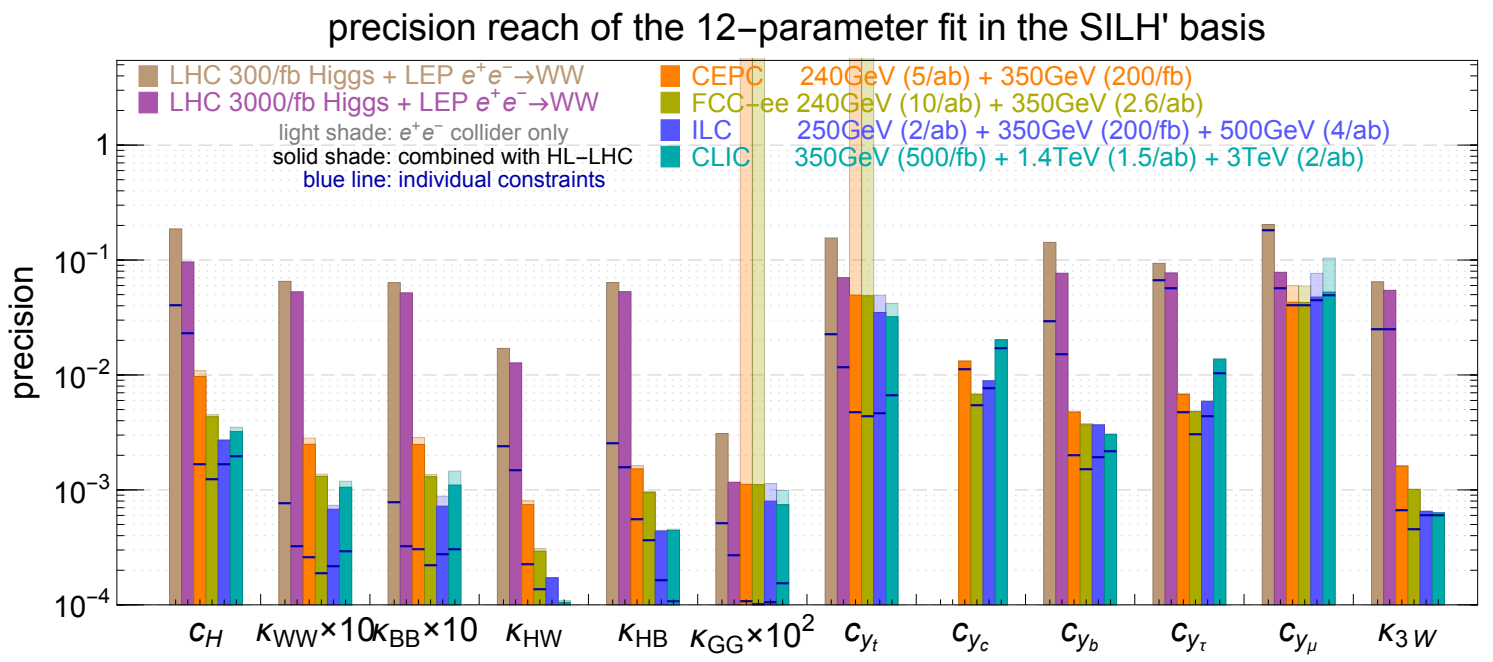

Figure 14. Same as figure 7 but is in the SILH'(-like) basis defined by eq. (A.8) and table 1.

here for the FCC-ee and ILC in figure 15-21. In particular, figure 16 shows the precision reach for ILC with different scenarios including runs at $250 \mathrm{GeV}, 350 \mathrm{GeV}$ and $500 \mathrm{GeV}$, while figure 17 further shows the potential improvement with the inclusion of a $1 \mathrm{TeV}$ run.

\section{Numerical expressions for the observables}

We express some of the important observables as numerical functions of the parameters in eq. (2.1), which is fed into the chi-square in eqs. (3.5)-(3.7). The SM input parameters we use in our analytical expressions are $G_{F}=1.1663787 \times 10^{-5} \mathrm{GeV}^{-2}, m_{Z}=91.1876 \mathrm{GeV}$, 


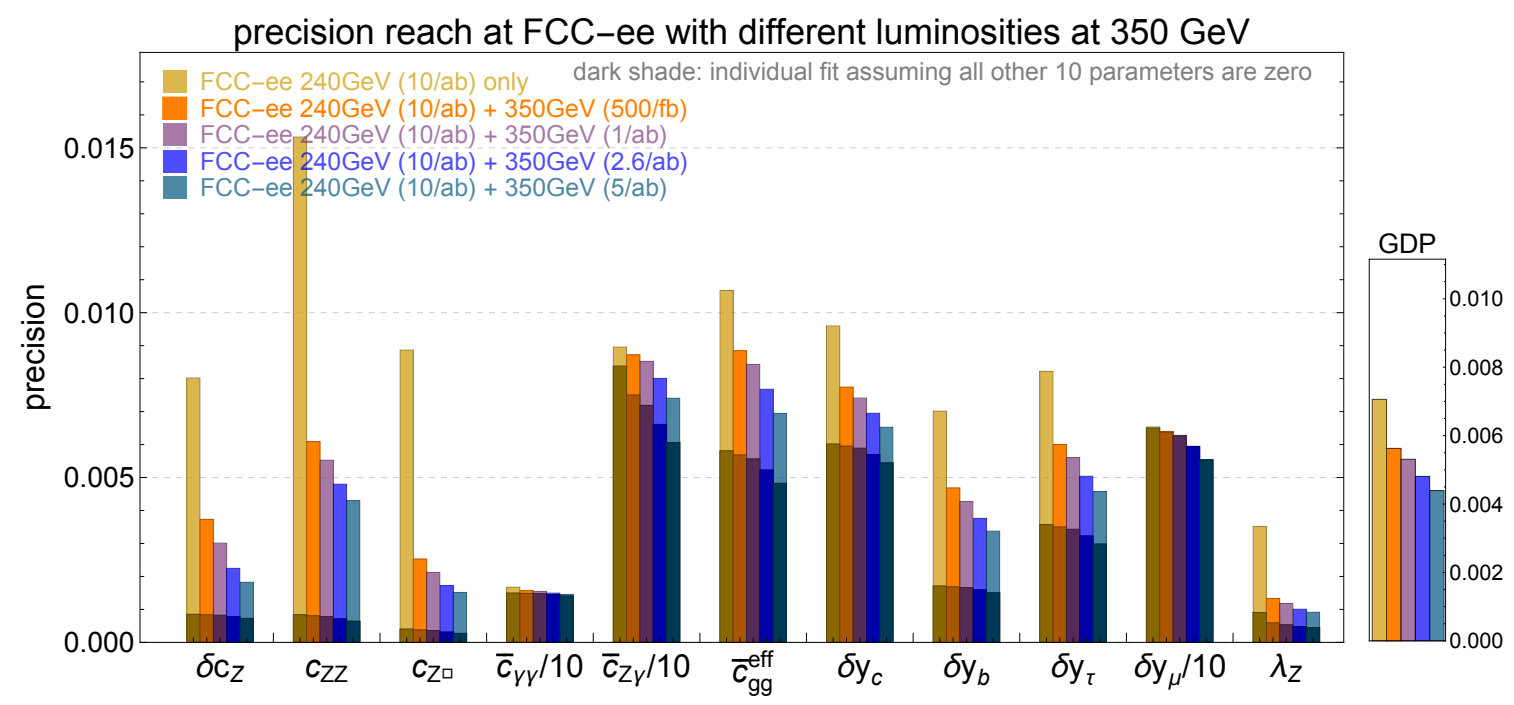

Figure 15. Same as figure 9 but for FCC-ee with $10 \mathrm{ab}^{-1}$ at $240 \mathrm{GeV}$ and $500 \mathrm{fb}^{-1}, 1 \mathrm{ab}^{-1}, 2.6 \mathrm{ab}^{-1}$ and $5 \mathrm{ab}^{-1}$.

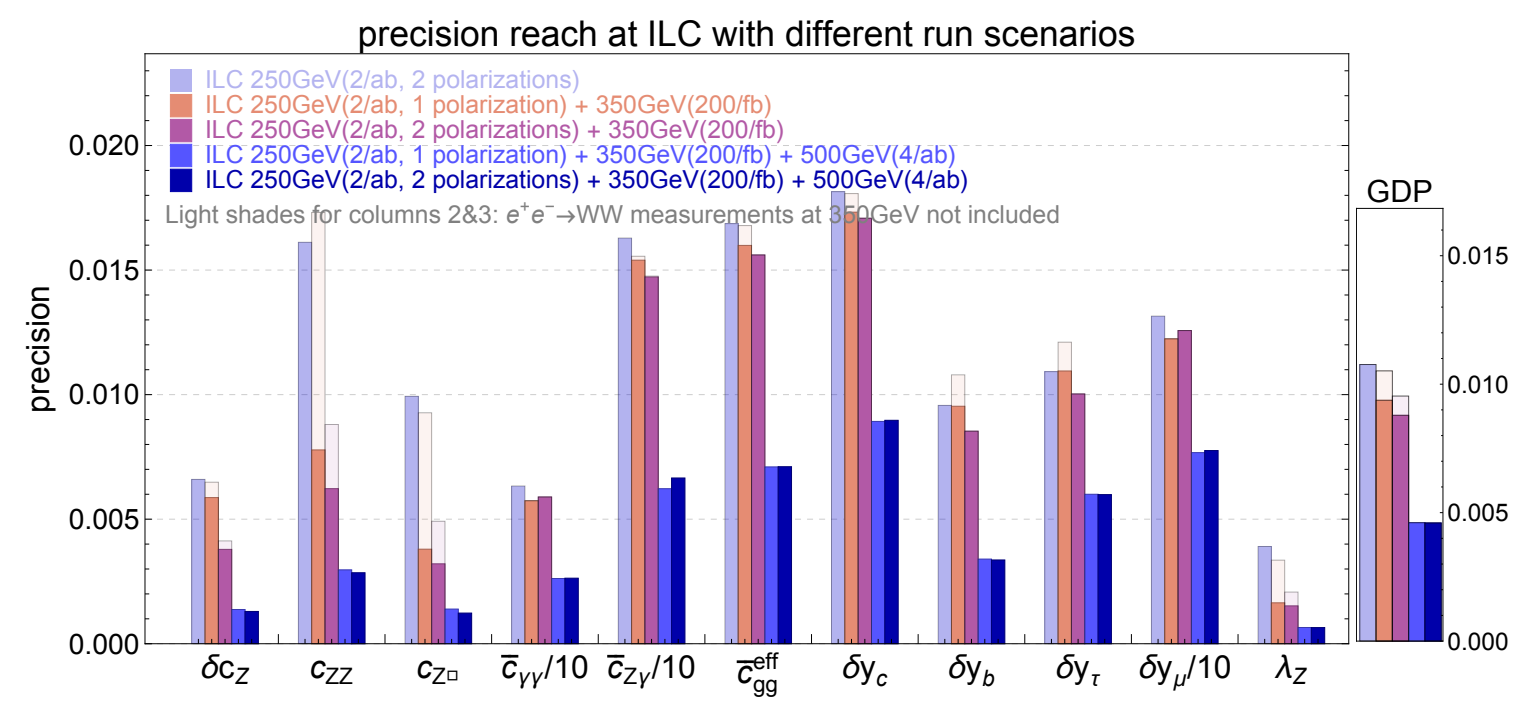

Figure 16. The precision reach for ILC with different scenarios. The 1st column corresponds to the ILC $250 \mathrm{GeV}$ run with $2 \mathrm{ab}^{-1}$ luminosity which is divided into two runs with polarizations $P\left(e^{-}, e^{+}\right)=(-0.8,+0.3)$ and $(+0.8,-0.3)$, and fractions 0.7 and 0.3 , respectively (see figure 10). The 2nd and 3rd columns include ILC $250 \mathrm{GeV}\left(2 \mathrm{ab}^{-1}\right)$ and $350 \mathrm{GeV}\left(200 \mathrm{fb}^{-1}\right)$. For the 2nd column, only the $(-0.8,+0.3)$ polarization is used for the $240 \mathrm{GeV}$ run, while for the 3 rd column the $240 \mathrm{GeV}$ run is divided in the same way as for the 1st column. The results of the ILC full run $\left(2 \mathrm{ab}^{-1}\right.$ at $250 \mathrm{GeV}, 200 \mathrm{fb}^{-1}$ at $350 \mathrm{GeV}$ and $4 \mathrm{ab}^{-1}$ at $\left.500 \mathrm{GeV}\right)$ are shown in the 4 th and 5 th columns, while single polarization (two polarizations) at $250 \mathrm{GeV}$ has been assumed for the 4 th (5th) column, analogous to the 2 nd and 3rd columns. $P\left(e^{-}, e^{+}\right)=(-0.8,+0.3)$ is assumed for the $350 \mathrm{GeV}$ and $500 \mathrm{GeV}$ runs. We found that dividing the runs at $350 \mathrm{GeV}$ and $500 \mathrm{GeV}$ into multiple polarization does not improve the results. For the ILC full program, we still show the constraint of $\bar{c}_{g g}^{\mathrm{eff}}$ instead of $c_{g g}$ and $\delta y_{t}$ in order to compare with other scenarios. For the full program only the $500 \mathrm{GeV}$ TGC results are used for consistency with the main results in figure 7. 


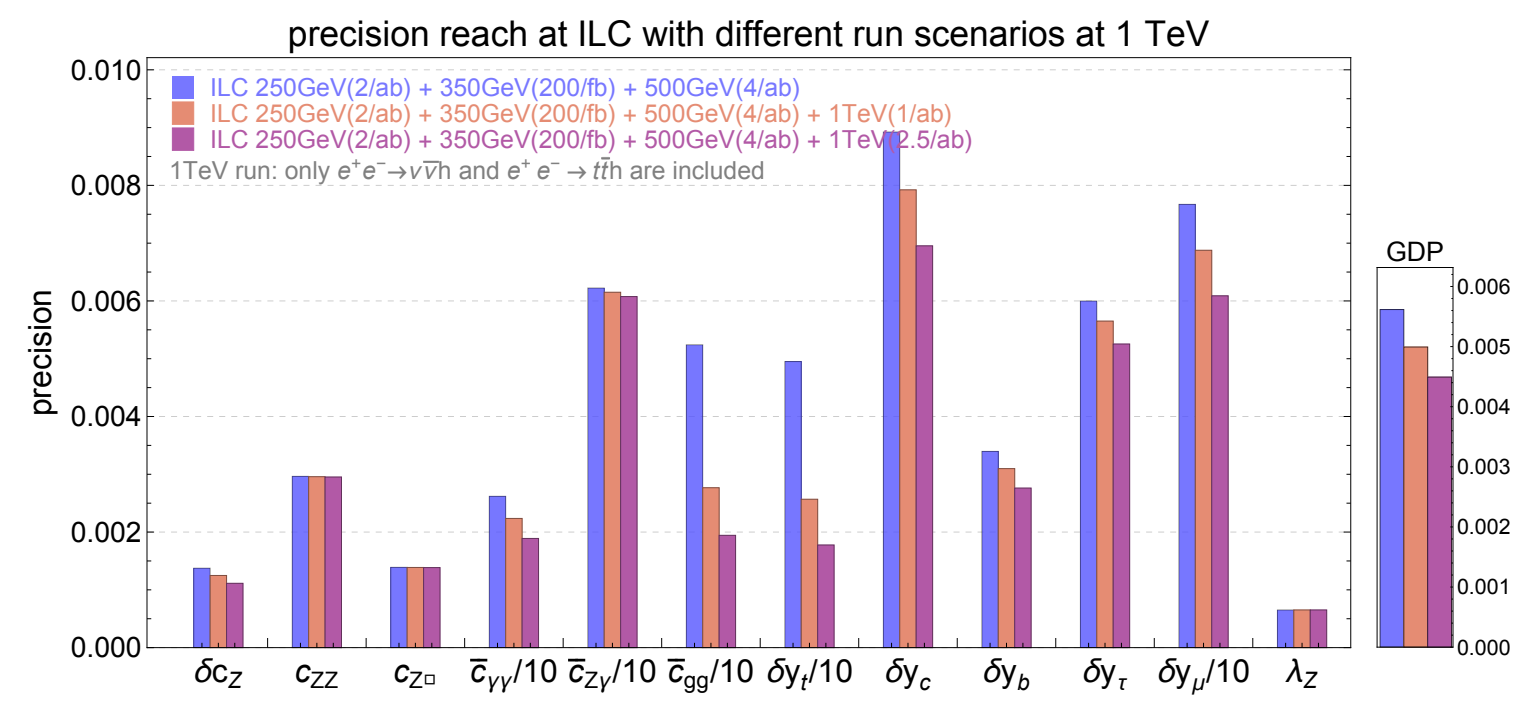

Figure 17. The precision reach for ILC with different scenarios for the $1 \mathrm{TeV}$ run. The 1st column corresponds to the ILC full run considered in our study with $2 \mathrm{ab}^{-1}$ at $250 \mathrm{GeV}, 200 \mathrm{fb}^{-1}$ at $350 \mathrm{GeV}$ and $4 \mathrm{ab}^{-1}$ at $500 \mathrm{GeV}$ and a fixed polarization of $P\left(e^{-}, e^{+}\right)=(-0.8,+0.3)$. For the 2nd (3rd) column, an additional run at $1 \mathrm{TeV}$ with an integrated luminosity of $1 \mathrm{ab}^{-1}\left(2.5 \mathrm{ab}^{-1}\right)$ and polarization $P\left(e^{-}, e^{+}\right)=(-0.8,+0.2)$ is also included. For the $1 \mathrm{TeV}$ run, the estimated measurement precisions in ref. [59] are used. Only the measurements of the $e^{+} e^{-} \rightarrow \nu \bar{\nu} h$ and $e^{+} e^{-} \rightarrow t \bar{t} h$ processes are included at $1 \mathrm{TeV}$, as the ones for $e^{+} e^{-} \rightarrow h Z$ and $e^{+} e^{-} \rightarrow W W$ are not provided. In particular, the precision of $\sigma(t \bar{t} h) \times \mathrm{BR}(h \rightarrow b \bar{b})$ at $1 \mathrm{TeV}$ is estimated to be $6.0 \%$ with $1 \mathrm{ab}^{-1}$ data and $3.8 \%$ with $2.5 \mathrm{ab}^{-1}$ data, which significantly improves the precision at $500 \mathrm{GeV}$. As such, the constraints on both $\bar{c}_{g g}$ and $\delta y_{t}$ are greatly improved. It should be noted that the $e^{+} e^{-} \rightarrow h Z$ and $e^{+} e^{-} \rightarrow W W$ processes are more sensitive to some of the EFT parameters at higher energies. The inclusion of their measurements could potentially further improve the overall reach of the global fit.

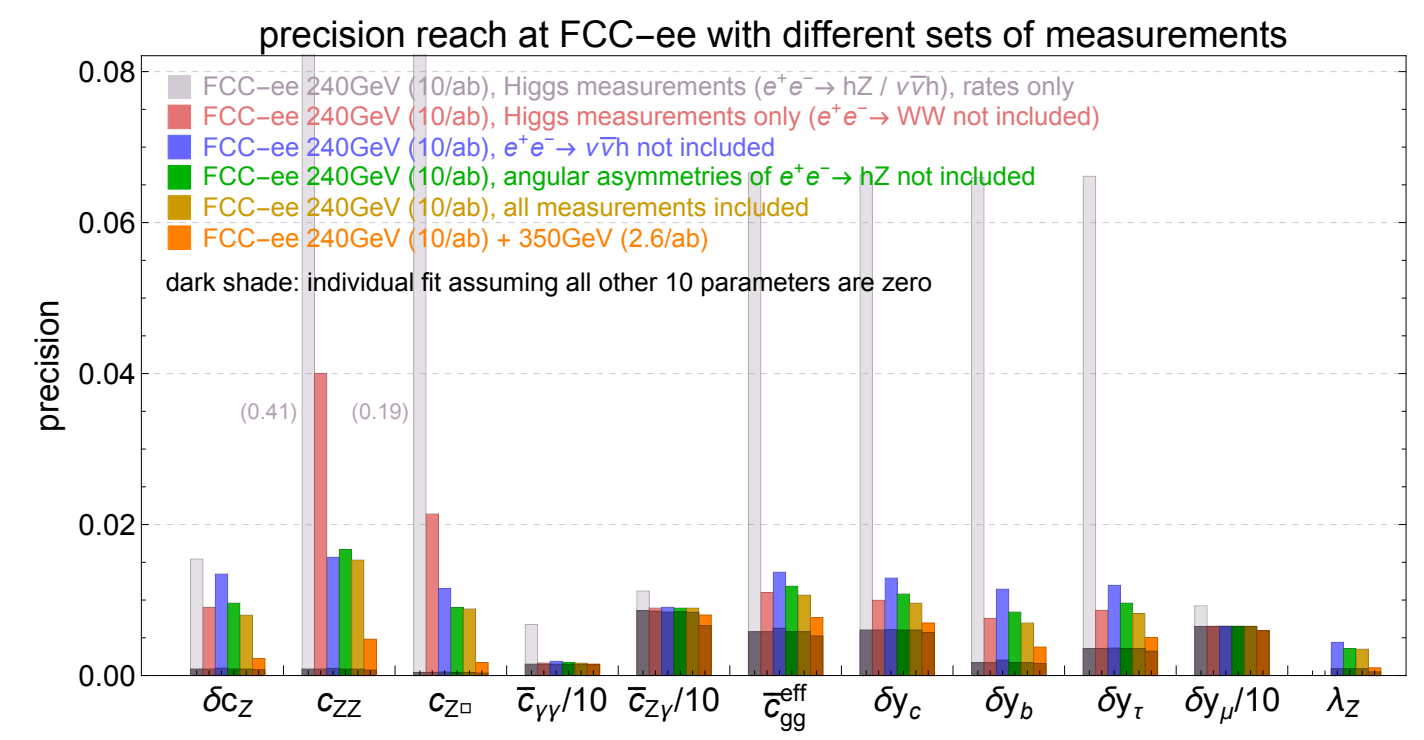

Figure 18. Same as figure 8 but for FCC-ee. 


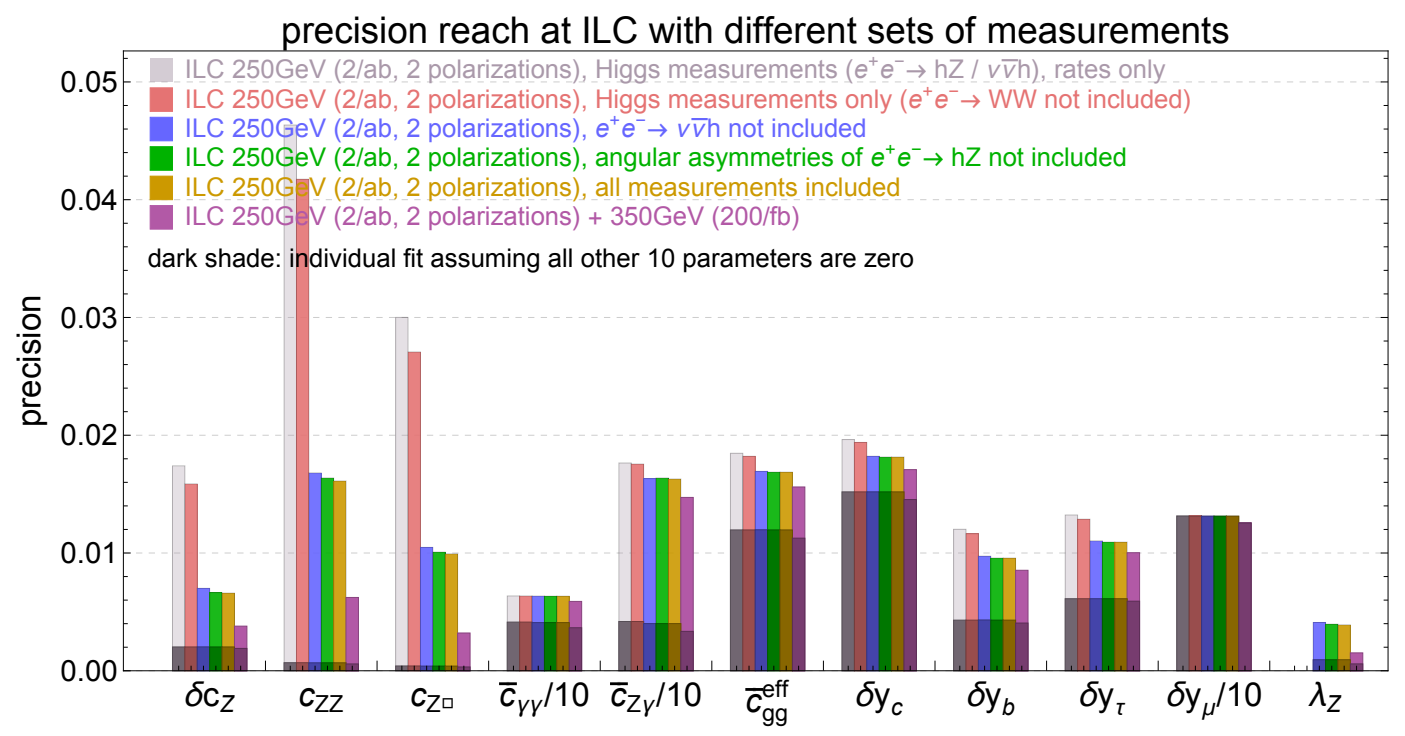

Figure 19. Same as figure 8 but for ILC with $2 \mathrm{ab}^{-1}$ at $250 \mathrm{GeV}$ and $200 \mathrm{fb}^{-1}$ at $350 \mathrm{GeV}$. The $250 \mathrm{GeV}$ run is divided into two runs with polarizations $P\left(e^{-}, e^{+}\right)=(-0.8,+0.3)$ and $(+0.8,-0.3)$, and fractions 0.7 and 0.3 , respectively (see figure 10).

$\alpha_{\mathrm{em}}\left(m_{Z}^{2}\right)=1 / 127.940$ and $m_{h}=125.09 \mathrm{GeV}$. For the rate of $e^{+} e^{-} \rightarrow h Z$, the measurements with the following energies and polarizations $P\left(e^{-}, e^{+}\right)$are used,

$\frac{\sigma_{h Z}}{\sigma_{h Z}^{\mathrm{SM}}}\left(\begin{array}{c}240 \mathrm{GeV} \text { unpolarized } \\ 250 \mathrm{GeV}(-0.8,+0.3) \\ 250 \mathrm{GeV}(+0.8,-0.3) \\ 350 \mathrm{GeV} \text { unpolarized } \\ 350 \mathrm{GeV}(-0.8,+0.3) \\ 500 \mathrm{GeV}(-0.8,+0.3) \\ 1.4 \mathrm{TeV} \text { unpolarized } \\ 3 \mathrm{TeV} \text { unpolarized }\end{array}\right) \simeq 1+2 \delta c_{Z}+\left(\begin{array}{c}1.8 \\ 5.6 \\ -2.9 \\ 2.8 \\ 11 \\ 21 \\ 14 \\ 52\end{array}\right) c_{Z Z}+\left(\begin{array}{c}3.7 \\ 9.8 \\ -3.2 \\ 7.5 \\ 20 \\ 41 \\ 115 \\ 526\end{array}\right) c_{Z \square}+\left(\begin{array}{c}-0.048 \\ -0.73 \\ 0.79 \\ -0.11 \\ -1.5 \\ -3.3 \\ -1.9 \\ -8.8\end{array}\right) c_{\gamma \gamma}+\left(\begin{array}{c}-0.087 \\ -1.3 \\ 1.5 \\ -0.24 \\ -3.3 \\ -8.1 \\ -5.5 \\ -26\end{array}\right) c_{Z \gamma}$.

As noted in section 3.1, the interferences between $s$-channel $Z$ and photon amplitudes are accidentally suppressed in the unpolarized total cross section. On the contrary, they have a significant impact when polarized beams are used, flipping for instance the sign of the $c_{Z Z}$ prefactor as polarization is reversed at $\sqrt{s}=250 \mathrm{GeV} .{ }^{11}$ The relevant expressions for

\footnotetext{
${ }^{11}$ For simplicity, the one-loop standard-model contributions to the $h Z_{\gamma}$ vertex are not included in the expressions above. They have a relatively large impact on the numerical prefactors of the $c_{\gamma \gamma}$ and $c_{Z \gamma}$ coefficients which are accidentally suppressed in the unpolarized cross section, at $240 \mathrm{GeV}$ in particular. Given that this measurement has little sensitivity to these coefficients, such contributions do however not affect the results of our global analysis. Note that the $c_{\gamma} \square$ parameter, directly related to the $h Z \gamma$ vertex, is written in terms of $c_{Z Z}, c_{Z \square}, c_{\gamma \gamma}$ and $c_{Z \gamma}$ using eq. (A.3).
} 

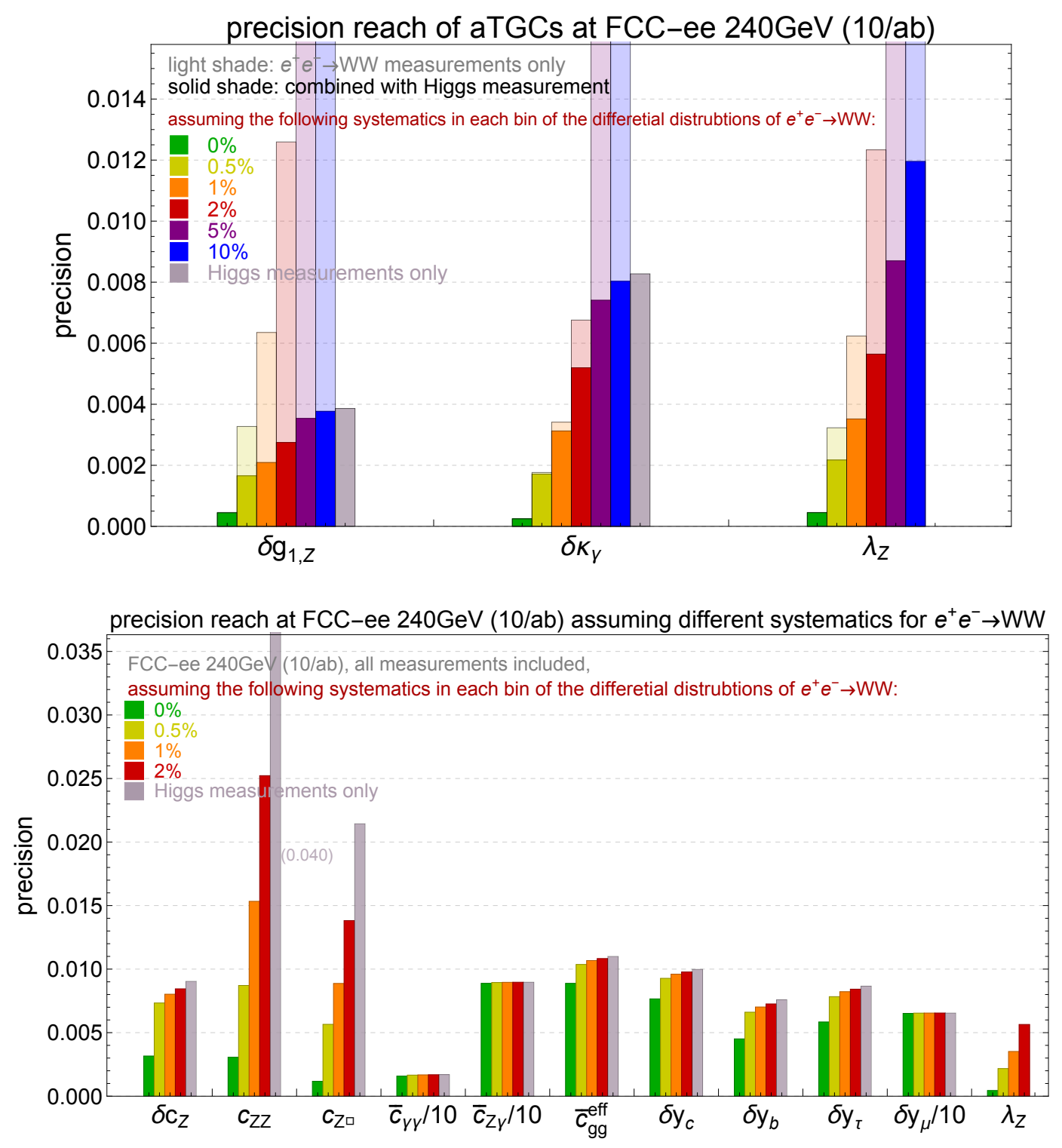

Figure 20. Same as figure 12 but for FCC-ee $240 \mathrm{GeV}$ with $10 \mathrm{ab}^{-1}$.

the WW fusion process are

$$
\frac{\sigma_{W W \rightarrow h}}{\sigma_{W W \rightarrow h}^{\mathrm{SM}}}\left(\begin{array}{c}
240 \mathrm{GeV} \\
250 \mathrm{GeV} \\
350 \mathrm{GeV} \\
500 \mathrm{GeV} \\
1 \mathrm{TeV} \\
1.4 \mathrm{TeV} \\
3 \mathrm{TeV}
\end{array}\right) \simeq 1+2 \delta c_{Z}+\left(\begin{array}{c}
-0.25 \\
-0.27 \\
-0.40 \\
-0.53 \\
-0.76 \\
-0.86 \\
-1.1
\end{array}\right) c_{Z Z}+\left(\begin{array}{c}
-0.68 \\
-0.72 \\
-1.1 \\
-1.5 \\
-2.2 \\
-2.5 \\
-3.4
\end{array}\right) c_{Z \square}+\left(\begin{array}{c}
0.035 \\
0.037 \\
0.056 \\
0.075 \\
0.12 \\
0.14 \\
0.18
\end{array}\right) c_{\gamma \gamma}+\left(\begin{array}{c}
0.090 \\
0.097 \\
0.14 \\
0.20 \\
0.32 \\
0.37 \\
0.52
\end{array}\right) c_{Z \gamma},
$$



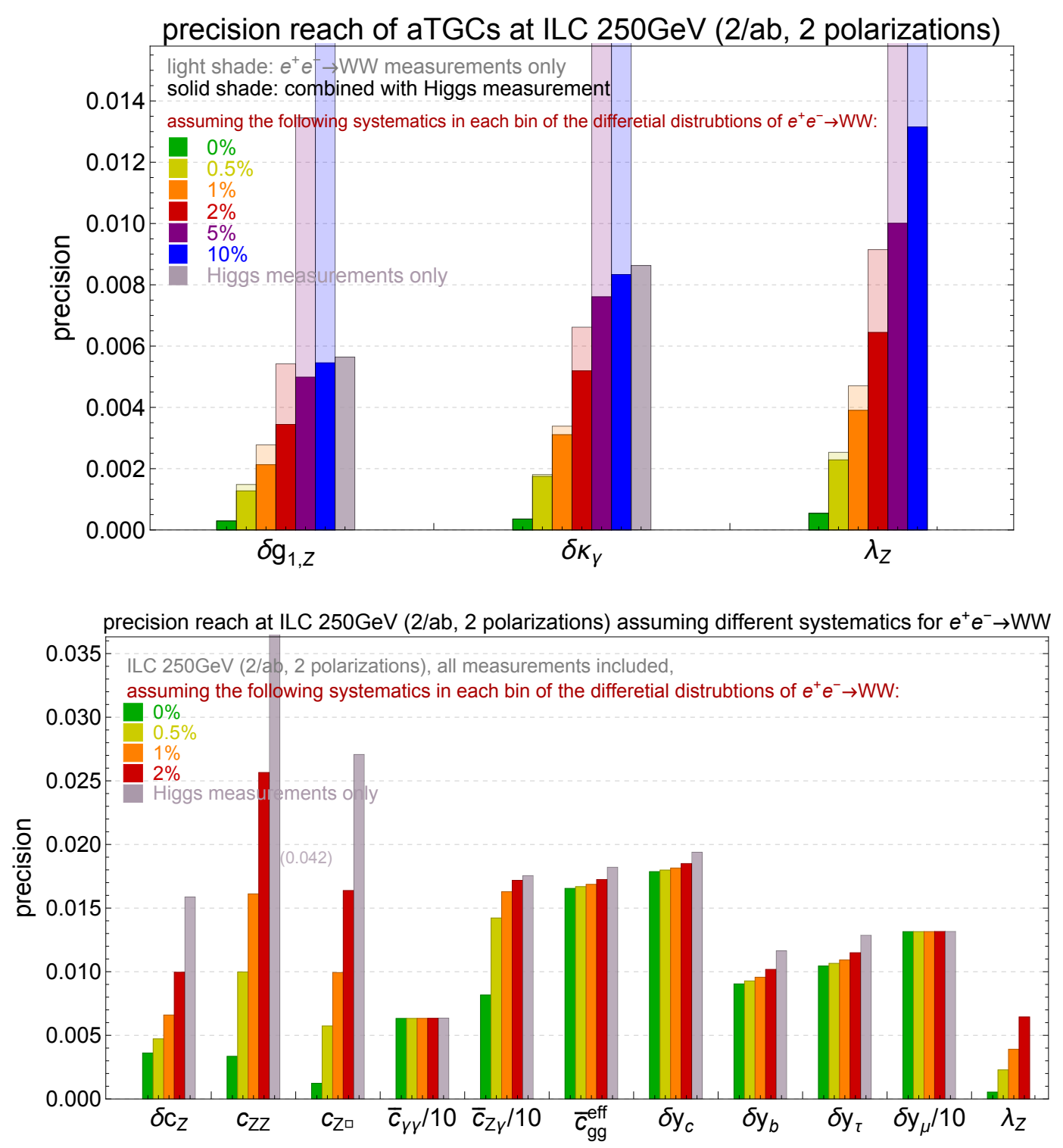

Figure 21. Same as figure 12 but for ILC $250 \mathrm{GeV}$ with $2 \mathrm{ab}^{-1}$ luminosity, divided into two runs with polarizations $P\left(e^{-}, e^{+}\right)=(-0.8,+0.3)$ and $(+0.8,-0.3)$, and fractions 0.7 and 0.3 , respectively (see figure 10).

which are obtained from MadGraph5 [88] with the BSMC model $[89,90]$ as functions of $\delta c_{W}, c_{W W}$ and $c_{W \square}$ and then transformed into the basis in eq. (2.1) with eq. (A.3). The default input parameters are used for these numerical computations. They apply to any polarizations since only the initial states with helicities $H\left(e^{-}, e^{+}\right)=(-,+)$contribute to this process.

For the $e^{+} e^{-} \rightarrow t \bar{t} h$ process, we only consider the dominate NP contribution which is from the modification of the top Yukawa, $\delta y_{t}$. It is therefore straight forward to write 
down the rate of the $t \bar{t} h$ process as

$$
\frac{\sigma_{t \bar{t} h}}{\sigma_{t \bar{t} h}^{\mathrm{SM}}} \simeq 1+2 \delta y_{t}
$$

For Higgs decays, we make use of the results in ref. [16]. The Decay widths to a pair of fermions are

$$
\frac{\Gamma_{c c}}{\Gamma_{c c}^{\mathrm{SM}}} \simeq 1+2 \delta y_{c}, \quad \frac{\Gamma_{b b}}{\Gamma_{b b}^{\mathrm{SM}}} \simeq 1+2 \delta y_{b}, \quad \frac{\Gamma_{\tau \tau}}{\Gamma_{\tau \tau}^{\mathrm{SM}}} \simeq 1+2 \delta y_{\tau}, \quad \frac{\Gamma_{\mu \mu}}{\Gamma_{\mu \mu}^{\mathrm{SM}}} \simeq 1+2 \delta y_{\mu} .
$$

The decay width to $W W^{*} Z Z^{*}$ (with $4 f$ final states) are given by

$$
\begin{aligned}
& \frac{\Gamma_{W W^{*}}}{\Gamma_{W W^{*}}^{\mathrm{SM}}} \simeq 1+2 \delta c_{Z}+0.05 c_{Z Z}+0.67 c_{Z \square}-0.05 c_{\gamma \gamma}-0.17 c_{Z \gamma}, \\
& \frac{\Gamma_{Z Z^{*}}}{\Gamma_{Z Z^{*}}^{\mathrm{SM}}} \simeq 1+2 \delta c_{Z}-0.15 c_{Z Z}+0.41 c_{Z \square},
\end{aligned}
$$

where we assume there is no NP correction to the gauge couplings of fermions. As stated in section 2, we do not consider contribution from off-shell photons that gives the same final states as $Z Z^{*}$, as they can be relatively easily removed by kinematic cuts.

The decay of Higgs to $g g, \gamma \gamma$ and $Z \gamma$ are generated at one-loop level in the SM. The leading EFT contribution could either be at tree level (which are generated in the UV theory by new particles in the loop) or come at loop level by modifying the couplings in the SM loops. As mentioned in section 2, we follow ref. [16] and include both the tree level EFT contribution $\left(c_{g g}\right)$ and the one-loop contribution (from $\delta y_{t}$ and $\delta y_{b}$ ) for $h \rightarrow g g$, while only keeping the tree level EFT contribution $\left(c_{\gamma \gamma}\right.$ and $\left.c_{Z \gamma}\right)$ for $h \rightarrow \gamma \gamma$ and $h \rightarrow Z \gamma$. The decay widths are given by ${ }^{12}$

$$
\frac{\Gamma_{g g}}{\Gamma_{g g}^{S M}} \simeq 1+241 c_{g g}+2.10 \delta y_{t}-0.10 \delta y_{b},
$$

and

$$
\begin{aligned}
& \frac{\Gamma_{\gamma \gamma}}{\Gamma_{\gamma \gamma}^{\mathrm{SM}}} \simeq\left(1+\frac{c_{\gamma \gamma}}{-8.3 \times 10^{-2}}\right)^{2}, \\
& \frac{\Gamma_{Z \gamma}}{\Gamma_{Z \gamma}^{\mathrm{SM}}} \simeq\left(1+\frac{c_{Z \gamma}}{-5.9 \times 10^{-2}}\right)^{2} .
\end{aligned}
$$

The branching ratio can be derived from the total decay width, which can be obtained from

$$
\frac{\Gamma_{\mathrm{tot}}}{\Gamma_{\mathrm{tot}}^{\mathrm{SM}}}=\sum_{i} \frac{\Gamma_{i}}{\Gamma_{i}^{\mathrm{SM}} \mathrm{Br}_{i}^{\mathrm{SM}}}
$$

In practice, one only needs to include the BSM effects of the main channels in the calculation of the total width. Finally, the physical observables in the form of $\sigma \times \mathrm{BR}$ can be constructed from the above information.

\footnotetext{
${ }^{12}$ The choices of the bottom mass value would change the numerical values in eq. (D.7), but has little impact on the global fit results.
} 
For the aTGCs, we also express eq. (A.6) numerically as

$$
\begin{aligned}
\delta g_{1, Z} & \simeq-0.120 c_{Z Z}-0.392 c_{Z \square}+0.0215 c_{\gamma \gamma}+0.0637 c_{Z \gamma}, \\
\delta \kappa_{\gamma} & \simeq 0.208 c_{Z Z}-0.0373 c_{\gamma \gamma}-0.11 c_{Z \gamma} .
\end{aligned}
$$

The expressions for the differential distributions in $e^{+} e^{-} \rightarrow W W$ for all energies and polarizations are too lengthy to be reported here. The necessary information can be conveniently reconstructed from the constraints on aTGCs in table 5, 6 and 7 .

Finally, the CP-even angular observables in $e^{+} e^{-} \rightarrow h Z$ are given by

$$
\begin{aligned}
& A_{\theta_{1}} \simeq\left(\begin{array}{l}
-0.45 \\
-0.46 \\
-0.46 \\
-0.57 \\
-0.57 \\
-0.65
\end{array}\right)+\left(\begin{array}{c}
0.050 \\
0.074 \\
0.074 \\
0.33 \\
0.33 \\
0.62
\end{array}\right) c_{Z Z}+\left(\begin{array}{c}
0.0019 \\
0.039 \\
-0.042 \\
0.013 \\
0.19 \\
0.37
\end{array}\right) c_{Z \gamma} \\
& A_{\phi}^{(3)} \simeq\left(\begin{array}{c}
0.0093 \\
0.069 \\
-0.065 \\
0.0092 \\
0.068 \\
0.058
\end{array}\right)+\left(\begin{array}{c}
0.32 \\
0.059 \\
0.10 \\
0.75 \\
0.15 \\
0.34
\end{array}\right) c_{Z Z}+\left(\begin{array}{c}
0.50 \\
0.096 \\
0.15 \\
1.14 \\
0.20 \\
0.36
\end{array}\right) c_{Z \square}+\left(\begin{array}{c}
-0.058 \\
-0.011 \\
-0.017 \\
-0.13 \\
-0.023 \\
-0.042
\end{array}\right) c_{\gamma \gamma}+\left(\begin{array}{c}
-0.11 \\
-0.023 \\
-0.034 \\
-0.28 \\
-0.036 \\
-0.025
\end{array}\right) c_{Z \gamma} \\
& A_{\phi}^{(4)} \simeq\left(\begin{array}{l}
0.096 \\
0.092 \\
0.092 \\
0.061 \\
0.061 \\
0.035
\end{array}\right)+\left(\begin{array}{c}
0.015 \\
0.022 \\
0.022 \\
0.098 \\
0.098 \\
0.19
\end{array}\right) c_{Z Z}+\left(\begin{array}{c}
0.00057 \\
0.012 \\
-0.013 \\
0.0040 \\
0.056 \\
0.11
\end{array}\right) c_{Z \gamma} \\
& A_{c \theta_{1}, c \theta_{2}} \simeq\left(\begin{array}{c}
-0.0052 \\
-0.037 \\
0.034 \\
-0.0033 \\
-0.024 \\
-0.014
\end{array}\right)+\left(\begin{array}{c}
-0.18 \\
-0.042 \\
-0.043 \\
-0.27 \\
-0.085 \\
-0.13
\end{array}\right) c_{Z Z}+\left(\begin{array}{c}
-0.28 \\
-0.050 \\
-0.078 \\
-0.40 \\
-0.070 \\
-0.086
\end{array}\right) c_{Z \square}+\left(\begin{array}{c}
0.032 \\
0.0059 \\
0.0092 \\
0.047 \\
0.0082 \\
0.010
\end{array}\right) c_{\gamma \gamma}+\left(\begin{array}{c}
0.054 \\
0.0051 \\
0.010 \\
0.074 \\
-0.0092 \\
-0.028
\end{array}\right) c_{Z \gamma}
\end{aligned}
$$

where the six entries in each column correspond to the center of energies and beam polarizations $P\left(e^{-}, e^{+}\right)$in the following order: $240 \mathrm{GeV}$ unpolarized, $250 \mathrm{GeV}$ $(-0.8,+0.3), 250 \mathrm{GeV}(+0.8,-0.3), 350 \mathrm{GeV}$ unpolarized, $350 \mathrm{GeV}(-0.8,+0.3)$ and $500 \mathrm{GeV}(-0.8,+0.3)$. 


\begin{tabular}{|c|c|c|c|c|}
\hline & \multicolumn{4}{|c|}{ precision (one standard deviation) } \\
\hline & CEPC & FCC-ee & ILC & CLIC \\
\hline$\delta c_{Z}$ & 0.00485 & 0.00216 & 0.00134 & 0.00161 \\
\hline$c_{Z Z}$ & 0.00701 & 0.00466 & 0.00291 & 0.00229 \\
\hline$c_{Z \square}$ & 0.00328 & 0.00171 & 0.00139 & 0.000215 \\
\hline$c_{\gamma \gamma}$ & 0.00130 & 0.000922 & 0.00115 & 0.00132 \\
\hline$c_{Z \gamma}$ & 0.00537 & 0.00406 & 0.00332 & 0.00426 \\
\hline$c_{g g}$ & 0.000430 & 0.000427 & 0.000307 & 0.000286 \\
\hline$\delta y_{t}$ & 0.0495 & 0.0489 & 0.0349 & 0.0322 \\
\hline$\delta y_{c}$ & 0.0139 & 0.00686 & 0.00890 & 0.0208 \\
\hline$\delta y_{b}$ & 0.00559 & 0.00359 & 0.00334 & 0.00311 \\
\hline$\delta y_{\tau}$ & 0.00769 & 0.00490 & 0.00592 & 0.0143 \\
\hline$\delta y_{\mu}$ & 0.0429 & 0.0427 & 0.0476 & 0.0525 \\
\hline$\lambda_{Z}$ & 0.00161 & 0.00101 & 0.000652 & 0.000632 \\
\hline \multicolumn{5}{|c}{} \\
\hline
\end{tabular}

Table 11. Precision reach (one standard deviation bounds) at each of the four future $e^{+} e^{-}$colliders. For each collider, the LHC $3000 \mathrm{fb}^{-1}$ (including $8 \mathrm{TeV}$ results) + LEP measurements are also combined in the total $\chi^{2}$.

\section{E Numerical results of the global fit}

We hereby list the numerical results of the global fit for the future $e^{+} e^{-}$colliders. The one standard deviation constraints on each of the 12 parameters in eq. (2.1) are listed in table 11, and the corresponding correlation matrices are shown in table 12-15. For each collider, the LHC $3000 \mathrm{fb}^{-1}$ (including $8 \mathrm{TeV}$ results) + LEP measurements are also combined in the total $\chi^{2}$, so that the results represent the "best reach" for each scenario. With this information, the corresponding chi-squared can be reconstructed using eq. (3.8), which can be used to constrain any particular model that satisfies the assumptions of the 12-parameter framework, where the 12 parameters in EFT are functions of a usually much smaller set of model parameters. To minimize the numerical uncertainties, three significant figures are provided for the one standard deviation constraints, which is likely more than sufficient for the level of precision of our estimations. For easy mapping to dimension- 6 operators and new physics models, we also switch back to the original definitions of $c_{\gamma \gamma}$, $c_{Z \gamma}$ and $c_{g g}$ (instead of $\bar{c}_{\gamma \gamma}, \bar{c}_{Z \gamma}$ and $\bar{c}_{g g}$ ). 


\begin{tabular}{|c|c|c|c|c|c|c|c|c|c|c|c|c|}
\hline \multicolumn{10}{|c|}{ correlation matrix, CEPC } \\
\hline & $\delta c_{Z}$ & $c_{Z Z}$ & $c_{Z \square}$ & $c_{\gamma \gamma}$ & $c_{Z \gamma}$ & $c_{g g}$ & $\delta y_{t}$ & $\delta y_{c}$ & $\delta y_{b}$ & $\delta y_{\tau}$ & $\delta y_{\mu}$ & $\lambda_{Z}$ \\
\hline$\delta c_{Z}$ & 1 & -0.37 & -0.39 & -0.072 & -0.15 & 0.023 & 0.041 & 0.31 & 0.60 & 0.50 & 0.050 & -0.21 \\
\hline$c_{Z Z}$ & & 1 & -0.69 & 0.083 & 0.34 & -0.028 & -0.015 & -0.19 & -0.44 & -0.33 & -0.040 & -0.72 \\
\hline$c_{Z \square}$ & & 1 & -0.0092 & -0.18 & 0.0094 & -0.022 & -0.063 & -0.11 & -0.11 & -0.0076 & 0.89 \\
\hline$c_{\gamma \gamma}$ & & & & 1 & 0.028 & -0.17 & 0.19 & -0.049 & -0.069 & -0.063 & -0.044 & -0.016 \\
\hline$c_{Z \gamma}$ & & & & & 1 & -0.014 & -0.011 & -0.11 & -0.25 & -0.18 & -0.021 & -0.11 \\
\hline$c_{g g}$ & & & & & & 1 & -0.99 & 0.014 & 0.035 & 0.025 & 0.16 & 0.011 \\
\hline$\delta y_{t}$ & & & & & & & 1 & 0.039 & 0.077 & 0.061 & -0.16 & -0.012 \\
\hline$\delta y_{c}$ & & & & & & & & 1 & 0.48 & 0.37 & 0.036 & -0.011 \\
\hline$\delta y_{b}$ & & & & & & & & 1 & 0.76 & 0.067 & -0.0012 \\
\hline$\delta y_{\tau}$ & & & & & & & & & & 1 & 0.054 & -0.019 \\
\hline$\delta y_{\mu}$ & & & & & & & & & & & 1 & 0.0017 \\
\hline$\lambda_{Z}$ & & & & & & & & & & & & 1 \\
\hline
\end{tabular}

Table 12. The corresponding correlation matrix for the CEPC one sigma bounds in table 11.

\begin{tabular}{|c|c|c|c|c|c|c|c|c|c|c|c|c|}
\hline \multicolumn{10}{|c|}{ correlation matrix, FCC-ee } \\
\hline & $\delta c_{Z}$ & $c_{Z Z}$ & $c_{Z \square}$ & $c_{\gamma \gamma}$ & $c_{Z \gamma}$ & $c_{g g}$ & $\delta y_{t}$ & $\delta y_{c}$ & $\delta y_{b}$ & $\delta y_{\tau}$ & $\delta y_{\mu}$ & $\lambda_{Z}$ \\
\hline$\delta c_{Z}$ & 1 & -0.49 & 0.073 & -0.055 & -0.22 & 0.026 & 0.0056 & 0.19 & 0.26 & 0.26 & 0.013 & 0.17 \\
\hline$c_{Z Z}$ & & 1 & -0.88 & 0.13 & 0.34 & -0.023 & -0.0025 & -0.24 & -0.44 & -0.33 & -0.027 & -0.81 \\
\hline$c_{Z \square}$ & & & 1 & -0.077 & -0.20 & 0.013 & -0.0018 & 0.13 & 0.24 & 0.15 & 0.015 & 0.86 \\
\hline$c_{\gamma \gamma}$ & & & 1 & 0.057 & -0.13 & 0.13 & -0.10 & -0.16 & -0.13 & -0.034 & -0.069 \\
\hline$c_{Z \gamma}$ & & & & & 1 & -0.013 & -0.0038 & -0.15 & -0.27 & -0.20 & -0.015 & -0.081 \\
\hline$c_{g g}$ & & & & & 1 & -0.99 & 0.026 & 0.049 & 0.036 & 0.16 & 0.012 \\
\hline$\delta y_{t}$ & & & & & & & 1 & 0.020 & 0.034 & 0.027 & -0.16 & 0.0008 \\
\hline$\delta y_{c}$ & & & & & & & 1 & 0.55 & 0.43 & 0.023 & 0.13 \\
\hline$\delta y_{b}$ & & & & & & & & & 1 & 0.75 & 0.038 & 0.25 \\
\hline$\delta y_{\tau}$ & & & & & & & & & 1 & 0.031 & 0.17 \\
\hline$\delta y_{\mu}$ & & & & & & & & & & & 1 & 0.016 \\
\hline$\lambda_{Z}$ & & & & & & & & & & & & 1 \\
\hline
\end{tabular}

Table 13. The corresponding correlation matrix for the FCC-ee one sigma bounds in table 11. 


\begin{tabular}{|c|c|c|c|c|c|c|c|c|c|c|c|c|}
\hline \multicolumn{10}{|c|}{ correlation matrix, ILC } \\
\hline & $\delta c_{Z}$ & $c_{Z Z}$ & $c_{Z \square}$ & $c_{\gamma \gamma}$ & $c_{Z \gamma}$ & $c_{g g}$ & $\delta y_{t}$ & $\delta y_{c}$ & $\delta y_{b}$ & $\delta y_{\tau}$ & $\delta y_{\mu}$ & $\lambda_{Z}$ \\
\hline$\delta c_{Z}$ & 1 & -0.40 & 0.27 & 0.039 & -0.26 & 0.020 & 0.0087 & 0.091 & -0.059 & 0.13 & -0.0085 & -0.069 \\
\hline$c_{Z Z}$ & & 1 & -0.89 & 0.071 & 0.36 & -0.027 & 0.0020 & -0.13 & -0.29 & -0.24 & -0.014 & 0.23 \\
\hline$c_{Z \square}$ & & & 1 & -0.020 & 0.08 & 0.019 & -0.00026 & 0.087 & 0.22 & 0.15 & 0.0094 & -0.25 \\
\hline$c_{\gamma \gamma}$ & & & 1 & -0.024 & -0.10 & 0.13 & -0.030 & -0.046 & -0.043 & -0.025 & 0.0061 \\
\hline$c_{Z \gamma}$ & & & & & 1 & -0.0049 & -0.013 & -0.084 & -0.17 & -0.20 & -0.0066 & 0.0096 \\
\hline$c_{g g}$ & & & & & 1 & -0.98 & 0.029 & 0.081 & 0.046 & 0.12 & -0.0049 \\
\hline$\delta y_{t}$ & & & & & & 1 & 0.014 & 0.022 & 0.019 & -0.13 & -0.0000 \\
\hline$\delta y_{c}$ & & & & & & & & 1 & 0.40 & 0.26 & 0.013 & -0.023 \\
\hline$\delta y_{b}$ & & & & & & & & 1 & 0.60 & 0.024 & -0.058 \\
\hline$\delta y_{\tau}$ & & & & & & & & & & 1 & 0.019 & -0.039 \\
\hline$\delta y_{\mu}$ & & & & & & & & & & & 1 & -0.0025 \\
\hline$\lambda_{Z}$ & & & & & & & & & & & & 1 \\
\hline
\end{tabular}

Table 14. The corresponding correlation matrix for the ILC one sigma bounds in table 11.

\begin{tabular}{|l|c|c|c|c|c|c|c|c|c|c|c|c|}
\hline \multicolumn{10}{|c|}{ correlation matrix, CLIC } \\
\hline & $\delta c_{Z}$ & $c_{Z Z}$ & $c_{Z \square}$ & $c_{\gamma \gamma}$ & $c_{Z \gamma}$ & $c_{g g}$ & $\delta y_{t}$ & $\delta y_{c}$ & $\delta y_{b}$ & $\delta y_{\tau}$ & $\delta y_{\mu}$ & $\lambda_{Z}$ \\
\hline$\delta c_{Z}$ & 1 & -0.0065 & -0.14 & 0.089 & -0.17 & 0.088 & 0.048 & 0.36 & 0.30 & 0.49 & -0.019 & -0.013 \\
\hline$c_{Z Z}$ & & 1 & -0.46 & 0.051 & 0.73 & -0.013 & -0.0070 & -0.054 & -0.13 & -0.083 & -0.0014 & -0.0083 \\
\hline$c_{Z \square}$ & & & 1 & 0.022 & 0.14 & -0.0014 & 0.0064 & 0.0027 & -0.073 & 0.0050 & -0.0054 & 0.020 \\
\hline$c_{\gamma \gamma}$ & & & 1 & -0.023 & -0.041 & 0.14 & -0.032 & 0.013 & -0.052 & -0.043 & 0.0032 \\
\hline$c_{Z \gamma}$ & & & & & 1 & -0.012 & -0.015 & -0.056 & -0.22 & -0.084 & -0.0033 & 0.027 \\
\hline$c_{g g}$ & & & & & 1 & -0.95 & 0.013 & 0.10 & 0.016 & 0.11 & -0.0004 \\
\hline$\delta y_{t}$ & & & & & & 1 & 0.023 & 0.035 & 0.031 & -0.13 & 0.0005 \\
\hline$\delta y_{c}$ & & & & & & & & 1 & 0.30 & 0.095 & 0.011 & -0.0008 \\
\hline$\delta y_{b}$ & & & & & & & & 1 & 0.42 & 0.0057 & -0.0082 \\
\hline$\delta y_{\tau}$ & & & & & & & & & & 1 & 0.016 & -0.0012 \\
\hline$\delta y_{\mu}$ & & & & & & & & & & & 1 & -0.0005 \\
\hline$\lambda_{Z}$ & & & & & & & & & & & & 1 \\
\hline
\end{tabular}

Table 15. The corresponding correlation matrix for the CLIC one sigma bounds in table 11.

Open Access. This article is distributed under the terms of the Creative Commons Attribution License (CC-BY 4.0), which permits any use, distribution and reproduction in any medium, provided the original author(s) and source are credited.

\section{References}

[1] CEPC-SPPC Study Group collaboration, CEPC-SPPC preliminary conceptual design report 1. Physics and detector, CEPC, China, (2015).

[2] Tlep Design Study Working Group collaboration, M. Bicer et al., First look at the physics case of TLEP, JHEP 01 (2014) 164 [arXiv:1308.6176] [INSPIRE]. 
[3] H. Baer et al., The International Linear Collider technical design report - volume 2: physics, arXiv: 1306.6352 [INSPIRE].

[4] CLICDP and CLIC collaborations, M.J. Boland et al., Updated baseline for a staged Compact Linear Collider, arXiv:1608.07537 [INSPIRE].

[5] LHC Higgs Cross section Working Group collaboration, A. David et al., LHC $H X S W G$ interim recommendations to explore the coupling structure of a Higgs-like particle, arXiv: 1209.0040 [INSPIRE].

[6] W. Buchmüller and D. Wyler, Effective Lagrangian analysis of new interactions and flavor conservation, Nucl. Phys. B 268 (1986) 621 [INSPIRE].

[7] G.F. Giudice, C. Grojean, A. Pomarol and R. Rattazzi, The strongly-interacting light Higgs, JHEP 06 (2007) 045 [hep-ph/0703164] [INSPIRE].

[8] B. Grzadkowski, M. Iskrzynski, M. Misiak and J. Rosiek, Dimension-six terms in the Standard Model Lagrangian, JHEP 10 (2010) 085 [arXiv: 1008.4884] [INSPIRE].

[9] R. Contino, M. Ghezzi, C. Grojean, M. Muhlleitner and M. Spira, Effective Lagrangian for a light Higgs-like scalar, JHEP 07 (2013) 035 [arXiv:1303.3876] [INSPIRE].

[10] B. Henning, X. Lu and H. Murayama, How to use the Standard Model effective field theory, JHEP 01 (2016) 023 [arXiv: 1412.1837] [INSPIRE].

[11] LhC Higgs Cross section Working Group collaboration, D. de Florian et al., Handbook of LHC Higgs cross sections: 4. Deciphering the nature of the Higgs sector, arXiv: 1610.07922 [INSPIRE].

[12] A. Kobach, Baryon number, lepton number and operator dimension in the Standard Model, Phys. Lett. B 758 (2016) 455 [arXiv: 1604.05726] [INSPIRE].

[13] J. Elias-Miro, J.R. Espinosa, E. Masso and A. Pomarol, Higgs windows to new physics through $D=6$ operators: constraints and one-loop anomalous dimensions, JHEP 11 (2013) 066 [arXiv:1308.1879] [INSPIRE].

[14] A. Pomarol and F. Riva, Towards the ultimate SM fit to close in on Higgs physics, JHEP 01 (2014) 151 [arXiv: 1308.2803] [INSPIRE].

[15] J. Ellis, V. Sanz and T. You, Complete Higgs sector constraints on dimension-6 operators, JHEP 07 (2014) 036 [arXiv: 1404.3667] [INSPIRE].

[16] A. Falkowski, Effective field theory approach to LHC Higgs data, Pramana 87 (2016) 39 [arXiv: 1505.00046] [INSPIRE].

[17] A. Butter, O.J.P. Éboli, J. Gonzalez-Fraile, M.C. Gonzalez-Garcia, T. Plehn and M. Rauch, The gauge-Higgs legacy of the LHC run I, JHEP 07 (2016) 152 [arXiv: 1604.03105] [INSPIRE].

[18] Z. Han and W. Skiba, Effective theory analysis of precision electroweak data, Phys. Rev. D 71 (2005) 075009 [hep-ph/0412166] [INSPIRE].

[19] M. Ciuchini, E. Franco, S. Mishima and L. Silvestrini, Electroweak precision observables, new physics and the nature of a $126 \mathrm{GeV}$ Higgs boson, JHEP 08 (2013) 106 [arXiv:1306.4644] [INSPIRE]. 
[20] M. Ciuchini, E. Franco, S. Mishima, M. Pierini, L. Reina and L. Silvestrini, Update of the electroweak precision fit, interplay with Higgs-boson signal strengths and model-independent constraints on new physics, Nucl. Part. Phys. Proc. 273-275 (2016) 2219 [arXiv:1410.6940] [INSPIRE].

[21] A. Falkowski and F. Riva, Model-independent precision constraints on dimension-6 operators, JHEP 02 (2015) 039 [arXiv: 1411.0669] [INSPIRE].

[22] A. Efrati, A. Falkowski and Y. Soreq, Electroweak constraints on flavorful effective theories, JHEP 07 (2015) 018 [arXiv: 1503.07872] [INSPIRE].

[23] A. Falkowski, M. Gonzalez-Alonso, A. Greljo and D. Marzocca, Global constraints on anomalous triple gauge couplings in effective field theory approach, Phys. Rev. Lett. 116 (2016) 011801 [arXiv:1508.00581] [INSPIRE].

[24] A. Falkowski, M. Gonzalez-Alonso, A. Greljo, D. Marzocca and M. Son, Anomalous triple gauge couplings in the effective field theory approach at the LHC, JHEP 02 (2017) 115 [arXiv: 1609.06312] [INSPIRE].

[25] Z. Zhang, Time to go beyond triple-gauge-boson-coupling interpretation of $W$ pair production, Phys. Rev. Lett. 118 (2017) 011803 [arXiv:1610.01618] [INSPIRE].

[26] J. Ellis, V. Sanz and T. You, The effective Standard Model after LHC run I, JHEP 03 (2015) 157 [arXiv:1410.7703] [INSPIRE].

[27] L. Berthier and M. Trott, Consistent constraints on the Standard Model effective field theory, JHEP 02 (2016) 069 [arXiv: 1508.05060] [INSPIRE].

[28] N. Craig, M. Farina, M. McCullough and M. Perelstein, Precision Higgsstrahlung as a probe of new physics, JHEP 03 (2015) 146 [arXiv:1411.0676] [INSPIRE].

[29] M. Beneke, D. Boito and Y.-M. Wang, Anomalous Higgs couplings in angular asymmetries of $H \rightarrow Z \ell^{+} \ell^{-}$and $e^{+} e^{-} \rightarrow H Z$, JHEP 11 (2014) 028 [arXiv:1406.1361] [InSPIRE].

[30] B. Henning, X. Lu and H. Murayama, What do precision Higgs measurements buy us?, arXiv: 1404.1058 [INSPIRE].

[31] N. Craig, J. Gu, Z. Liu and K. Wang, Beyond Higgs couplings: probing the Higgs with angular observables at future $e^{+} e^{-}$colliders, JHEP 03 (2016) 050 [arXiv:1512.06877] [INSPIRE].

[32] J. Ellis and T. You, Sensitivities of prospective future $e^{+} e^{-}$colliders to decoupled new physics, JHEP 03 (2016) 089 [arXiv: 1510.04561] [INSPIRE].

[33] S.-F. Ge, H.-J. He and R.-Q. Xiao, Probing new physics scales from Higgs and electroweak observables at $e^{+} e^{-}$Higgs factory, JHEP 10 (2016) 007 [arXiv: 1603.03385] [INSPIRE].

[34] J. de Blas et al., Electroweak precision observables and Higgs-boson signal strengths in the Standard Model and beyond: present and future, JHEP 12 (2016) 135 [arXiv:1608.01509] [INSPIRE].

[35] J. Ellis, P. Roloff, V. Sanz and T. You, Dimension-6 operator analysis of the CLIC sensitivity to new physics, JHEP 05 (2017) 096 [arXiv:1701.04804] [INSPIRE].

[36] H. Khanpour and M. Mohammadi Najafabadi, Constraining Higgs boson effective couplings at electron-positron colliders, Phys. Rev. D 95 (2017) 055026 [arXiv: 1702.00951] [INSPIRE].

[37] D. Atwood and A. Soni, Analysis for magnetic moment and electric dipole moment form-factors of the top quark via $e^{+} e^{-} \rightarrow t \bar{t}$, Phys. Rev. D 45 (1992) 2405 [INSPIRE]. 
[38] M. Diehl and O. Nachtmann, Optimal observables for the measurement of three gauge boson couplings in $e^{+} e^{-} \rightarrow W^{+} W^{-}, Z$. Phys. C 62 (1994) 397 [inSPIRE].

[39] A. Falkowski, Higgs basis: proposal for an EFT basis choice for LHC HXSWG, LHCHXSWG-INT-2015-001, CERN, Geneva Switzerland, March 2015.

[40] S.M. Barr and A. Zee, Electric dipole moment of the electron and of the neutron, Phys. Rev. Lett. 65 (1990) 21 [Erratum ibid. 65 (1990) 2920] [INSPIRE].

[41] J. Fan and M. Reece, Probing charged matter through Higgs diphoton decay, gamma ray lines and EDMs, JHEP 06 (2013) 004 [arXiv: 1301.2597] [INSPIRE].

[42] ACME collaboration, J. Baron et al., Order of magnitude smaller limit on the electric dipole moment of the electron, Science 343 (2014) 269 [arXiv:1310.7534] [INSPIRE].

[43] Y.T. Chien, V. Cirigliano, W. Dekens, J. de Vries and E. Mereghetti, Direct and indirect constraints on CP-violating Higgs-quark and Higgs-gluon interactions, JHEP 02 (2016) 011 [arXiv: 1510.00725] [INSPIRE].

[44] R. Harnik, A. Martin, T. Okui, R. Primulando and F. Yu, Measuring CP-violation in $h \rightarrow \tau^{+} \tau^{-}$at colliders, Phys. Rev. D 88 (2013) 076009 [arXiv:1308.1094] [INSPIRE].

[45] A. Azatov, R. Contino, C.S. Machado and F. Riva, Helicity selection rules and noninterference for BSM amplitudes, Phys. Rev. D 95 (2017) 065014 [arXiv:1607.05236] [INSPIRE].

[46] F. Goertz, Indirect handle on the down-quark Yukawa coupling, Phys. Rev. Lett. 113 (2014) 261803 [arXiv: 1406.0102] [INSPIRE].

[47] W. Altmannshofer, J. Brod and M. Schmaltz, Experimental constraints on the coupling of the Higgs boson to electrons, JHEP 05 (2015) 125 [arXiv: 1503.04830] [INSPIRE].

[48] A.L. Kagan, G. Perez, F. Petriello, Y. Soreq, S. Stoynev and J. Zupan, Exclusive window onto Higgs Yukawa couplings, Phys. Rev. Lett. 114 (2015) 101802 [arXiv:1406.1722] [INSPIRE].

[49] C. Hartmann and M. Trott, Higgs decay to two photons at one loop in the Standard Model effective field theory, Phys. Rev. Lett. 115 (2015) 191801 [arXiv: 1507.03568] [INSPIRE].

[50] S. Di Vita, C. Grojean, G. Panico, M. Riembau and T. Vantalon, A global view on the Higgs self-coupling, arXiv:1704.01953 [INSPIRE].

[51] M. McCullough, An indirect model-dependent probe of the Higgs self-coupling, Phys. Rev. D 90 (2014) 015001 [arXiv:1312.3322] [INSPIRE].

[52] Y. Chen, R. Harnik and R. Vega-Morales, New opportunities in $h \rightarrow 4 \ell$, JHEP 09 (2015) 185 [arXiv: 1503.05855] [INSPIRE].

[53] S. Boselli, C.M. Carloni Calame, G. Montagna, O. Nicrosini, F. Piccinini and A. Shivaji, Higgs decay into four charged leptons in the presence of dimension-six operators, arXiv: 1703.06667 [INSPIRE].

[54] Z. Liu, L.-T. Wang and H. Zhang, Exotic decays of the $125 \mathrm{GeV}$ Higgs boson at future $e^{+} e^{-}$ lepton colliders, Chin. Phys. C 41 (2017) 063102 [arXiv:1612.09284] [INSPIRE].

[55] M. Ruan, Status \& updates from CEPC, in $1^{\text {st }}$ FCC Physics Workshop, CERN, Geneva Switzerland, (2017).

[56] M. Benedikt and F. Zimmermann, Future Circular Collider study, status and progress, in $1^{\text {st }}$ FCC Physics Workshop, CERN, Geneva Switzerland, (2017). 
[57] K. Fujii et al., Physics case for the International Linear Collider, arXiv:1506.05992 [INSPIRE].

[58] T. Barklow et al., ILC operating scenarios, arXiv:1506.07830 [INSPIRE].

[59] D.M. Asner et al., ILC Higgs white paper, in Proceedings, Community Summer Study 2013: Snowmass on the Mississippi (CSS2013), Minneapolis MN U.S.A., 29 July-6 August 2013 [arXiv: 1310.0763] [INSPIRE].

[60] H. Abramowicz et al., Higgs physics at the CLIC electron-positron linear collider, Eur. Phys. J. C 77 (2017) 475 [arXiv:1608.07538] [INSPIRE].

[61] D.R.T. Jones and S.T. Petcov, Heavy Higgs bosons at LEP, Phys. Lett. B 84 (1979) 440 [INSPIRE].

[62] P.S. Bhupal Dev, A. Djouadi, R.M. Godbole, M.M. Muhlleitner and S.D. Rindani, Determining the CP properties of the Higgs boson, Phys. Rev. Lett. 100 (2008) 051801 [arXiv:0707.2878] [INSPIRE].

[63] K. Hagiwara, S. Ishihara, R. Szalapski and D. Zeppenfeld, Low-energy effects of new interactions in the electroweak boson sector, Phys. Rev. D 48 (1993) 2182 [InSPIRE].

[64] G. Gounaris et al., Triple gauge boson couplings, in AGS/RHIC users annual meeting, Upton NY U.S.A., 15-16 June 1995, pg. 525 [hep-ph/9601233] [INSPIRE].

[65] DELPHI, OPAL, LEP Electroweak, ALEPH and L3 collaborations, S. Schael et al., Electroweak measurements in electron-positron collisions at $W$-boson-pair energies at LEP, Phys. Rept. 532 (2013) 119 [arXiv:1302.3415] [INSPIRE].

[66] Linear Collider American Working Group collaboration, T. Abe et al., Linear collider physics resource book for Snowmass 2001 — part 3: studies of exotic and Standard Model physics, hep-ex/0106057 [INSPIRE].

[67] CLIC Physics Working Group collaboration, E. Accomando et al., Physics at the CLIC multi-TeV linear collider, in Proceedings, $11^{\text {th }}$ International Conference on Hadron spectroscopy (Hadron 2005), Rio de Janeiro Brazil, 21-26 August 2005 [hep-ph/0412251] [INSPIRE].

[68] I. Marchesini, Triple gauge couplings and polarization at the ILC and leakage in a highly granular calorimeter, Ph.D. thesis, Hamburg U., Hamburg Germany, (2011).

[69] A. Rosca, Measurement of the charged triple gauge boson couplings at the ILC, Nucl. Part. Phys. Proc. 273-275 (2016) 2226 [INSPIRE].

[70] J.D. Wells and Z. Zhang, Status and prospects of precision analyses with $e^{+} e^{-} \rightarrow W^{+} W^{-}$, Phys. Rev. D 93 (2016) 034001 [arXiv: 1507.01594] [InSPIRE].

[71] L. Bian, J. Shu and Y. Zhang, Prospects for triple gauge coupling measurements at future lepton colliders and the $14 \mathrm{TeV}$ LHC, JHEP 09 (2015) 206 [arXiv:1507.02238] [INSPIRE].

[72] J. List, private communications, August 2017.

[73] ATLAS collaboration, Projections for measurements of Higgs boson signal strengths and coupling parameters with the ATLAS detector at a HL-LHC, ATL-PHYS-PUB-2014-016, CERN, Geneva Switzerland, (2014).

[74] ATLAS collaboration, Projections for measurements of Higgs boson cross sections, branching ratios and coupling parameters with the ATLAS detector at a HL-LHC, ATL-PHYS-PUB-2013-014, CERN, Geneva Switzerland, (2013). 
[75] ATLAS collaboration, HL-LHC projections for signal and background yield measurements of the $H \rightarrow \gamma \gamma$ when the Higgs boson is produced in association with $t$ quarks, $W$ or $Z$ bosons, ATL-PHYS-PUB-2014-012, CERN, Geneva Switzerland, (2014).

[76] ATLAS collaboration, Update of the prospects for the $H \rightarrow Z_{\gamma}$ search at the High-Luminosity LHC, ATL-PHYS-PUB-2014-006, CERN, Geneva Switzerland, (2014).

[77] ATLAS collaboration, Prospects for the study of the Higgs boson in the VH(bb) channel at HL-LHC, ATL-PHYS-PUB-2014-011, CERN, Geneva Switzerland, (2014).

[78] ATLAS collaboration, Studies of the VBF $H \rightarrow \tau_{\ell} \tau_{\text {had }}$ analysis at High Luminosity LHC conditions, ATL-PHYS-PUB-2014-018, CERN, Geneva Switzerland, (2014).

[79] R. Contino, A. Falkowski, F. Goertz, C. Grojean and F. Riva, On the validity of the effective field theory approach to SM precision tests, JHEP 07 (2016) 144 [arXiv:1604.06444] [INSPIRE].

[80] ATLAS collaboration, Search for the Standard Model Higgs and $Z$ boson decays to $J / \psi \gamma$ : HL-LHC projections, ATL-PHYS-PUB-2015-043, CERN, Geneva Switzerland, (2015).

[81] G.T. Bodwin, F. Petriello, S. Stoynev and M. Velasco, Higgs boson decays to quarkonia and

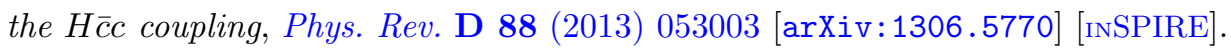

[82] G. Perez, Y. Soreq, E. Stamou and K. Tobioka, Constraining the charm Yukawa and Higgs-quark coupling universality, Phys. Rev. D 92 (2015) 033016 [arXiv:1503.00290] [INSPIRE].

[83] I. Brivio, F. Goertz and G. Isidori, Probing the charm quark Yukawa coupling in Higgs+charm production, Phys. Rev. Lett. 115 (2015) 211801 [arXiv:1507.02916] [INSPIRE].

[84] F. Bishara, U. Haisch, P.F. Monni and E. Re, Constraining light-quark Yukawa couplings from Higgs distributions, Phys. Rev. Lett. 118 (2017) 121801 [arXiv: 1606. 09253] [INSPIRE].

[85] L.M. Carpenter, T. Han, K. Hendricks, Z. Qian and N. Zhou, Higgs boson decay to light jets at the LHC, Phys. Rev. D 95 (2017) 053003 [arXiv:1611.05463] [INSPIRE].

[86] M. Ruan, Status \& updates from CEPC simulation - detector optimization, http://ias.ust.hk/program/shared_doc/2017/201701hep/HEP_20170124_Manqi_Ruan.pdf, (2017).

[87] T. Han, Z. Liu, Z. Qian and J. Sayre, Improving Higgs coupling measurements through ZZ fusion at the ILC, Phys. Rev. D 91 (2015) 113007 [arXiv:1504.01399] [InSPIRE].

[88] J. Alwall et al., The automated computation of tree-level and next-to-leading order differential cross sections and their matching to parton shower simulations, JHEP 07 (2014) 079 [arXiv: 1405.0301] [INSPIRE].

[89] B. Fuks and K. Mawatari, The BSM characterisation model, http://feynrules.irmp.ucl.ac.be/wiki/BSMCharacterisation, (2015).

[90] A. Falkowski, B. Fuks, K. Mawatari, K. Mimasu, F. Riva and V. sanz, Rosetta: an operator basis translator for Standard Model effective field theory, Eur. Phys. J. C 75 (2015) 583 [arXiv: 1508.05895] [INSPIRE]. 